\title{
Solving large interval availability models using a model transformation approach
}

\author{
Juan A. Carrasco* \\ Departament d'Enginyeria Electrònica, Universitat Politècnica de Catalunya, Diagonal 647, plta. 9 , \\ 08028 Barcelona, Spain
}

\begin{abstract}
Fault-tolerant systems are often modeled using (homogeneous) continuous time Markov chains (CTMCs). Computation of the distribution of the interval availability, i.e. of the distribution of the fraction of time in a time interval in which the system is operational, of a fault-tolerant system modeled by a CTMC is an important problem which has received attention recently. However, currently available methods to perform that computation are very expensive for large models and large time intervals. In this paper, we develop a new method to compute the distribution of the interval availability which, for large enough models and large enough time intervals, is significantly faster than previous methods. In the method, a truncated transformed model, which has with some arbitrarily small error the same interval availability distribution as the original model, is obtained from the original model and the truncated transformed model is solved using a previous state-of-the-art method. The method requires the selection of a "regenerative" state and its performance depends on that selection. For a class of models, including typical failure/repair models of coherent fault-tolerant systems with exponential failure and repair time distributions and repair in every state with failed components, a natural selection for the regenerative state exists and theoretical results are available assessing the performance of the method for that natural selection in terms of "visible" model characteristics. Those results can be used to anticipate when the method can be expected to be competitive for models in that class. Numerical results are presented showing that the new method can indeed be significantly faster than a previous state-of-the-art method and is able to deal with some large models and large time intervals in reasonable CPU times.
\end{abstract}

(C) 2003 Elsevier Ltd. All rights reserved.

Keywords: Fault-tolerant computer systems; Continuous time Markov chains; Interval availability distribution; Randomization; Model transformation

* Tel.: +34-93-4016652; fax: +34-93-4017785.

E-mail address: carrasco@eel.upc.es (J.A. Carrasco). 


\section{Introduction}

Fault-tolerant systems are often modeled using (homogeneous) continuous time Markov chains (CTMCs). Many fault-tolerant systems can be seen as operational, i.e. performing properly, or down, i.e. not performing at all, and dependability measures are appropriate to quantify those systems. The distribution of the interval availability, i.e. the distribution of the fraction of time in a time interval in which the system is operational, is a dependability measure of both practical and theoretical interest. This is mainly because that distribution tends to achieve its asymptotic shape (interval availability equal to the steady-state availability with probability one) very slowly and, then, the steady-state availability may be a poor measure of the behavior of the system over a finite time interval. Computing the interval availability distribution of a fault-tolerant system modeled by a CTMC is a challenging problem for which methods have been developed quite recently [1-9]. The first effort is reported in [1], where a closed form integral expression is obtained for a two-state model. In [3], randomization is used to obtain the distribution of the operational time in a time interval of the same two-state model. The first method able to deal with general finite CTMC models was developed by de Souza e Silva and Gail using randomization [9]. Goyal and Tantawi [2] developed a numerical approximate method, but they did not obtain bounds for the approximation error. Sericola [7] developed a closed form solution in terms of growing size matrices. Rubino and Sericola [4] developed an efficient algorithm to compute the distribution of the interval availability for the particular case in which operational and down periods are independent one by one and of each other, which is based on the analysis of the distributions of the durations of consecutive operational periods. In [5], Rubino and Sericola developed two algorithms which reduce the computational requirements of the randomization-based method developed in [9]. The first of such algorithms reduces the time requirements; the second one reduces the storage requirements. This second algorithm was reviewed in [6] as algorithm A, where it was taken as starting point to develop another algorithm (algorithm B), which is competitive when the number of operational states of the model is small and, furthermore, can deal with some class of CTMC models with denumerable infinite state spaces. In [8] closed-form expressions for the distribution of the occupation time in a subset of a CTMC were reviewed and those expressions were generalized to the joint distribution of the occupation times in different subsets. All currently available general methods for the computation of the distribution of the interval availability using CTMCs are computationally very expensive for large models and large time intervals, making of great interest the development of more efficient methods able to deal with large models and large time intervals in reasonable CPU times.

In this paper, we will consider a particular but quite wide class of CTMC models and will develop a new method for the computation of the interval availability distribution for models of that class. As we shall illustrate, the method will be able to deal in reasonable CPU times with some large models of that class and large time intervals. Formally, we will consider CTMCs with finite state space $\Omega=S$ or $\Omega=S \cup\{f\},|S| \geqslant 2$, where $f$ is an absorbing state, and will assume that (C1) either all states in $S$ are transient or $X$ has a single trapping component $C \subset S^{1}$ and (C2) all states

\footnotetext{
${ }^{1}$ Two states of a CTMC $i, j$ are strongly connected if there are paths in the state transition diagram of the CTMC from $i$ to $j$ and from $j$ to $i$; a state is strongly connected with itself; a component is a maximal subset of strongly connected states; a component is trapping if no state of the component has transition rates to states outside the component. States in non-trapping components are transient.
} 
are reachable (from some state with nonnull initial probability). The method requires the selection of a regenerative state $r \in S$. It will also be assumed that (C3) if $X$ has a single trapping component $C \subset S, r \in C .^{2}$ The set of states $\Omega$ is assumed partitioned into two subsets: the set of operational (up) states $U$ and the set of down states $D$. It will be assumed $U \neq \emptyset$ and $D \neq \emptyset$. The interval availability at time $t, \operatorname{IAV}(t)$, is defined as the fraction of time in the time interval $[0, t]$ in which the system is operational (up), i.e.

$$
\operatorname{IAV}(t)=\frac{1}{t} \int_{0}^{t} I_{X(\tau) \in U} \mathrm{~d} \tau
$$

where $I_{c}$ denotes the indicator function returning the value 1 if condition $c$ is satisfied and the value 0 otherwise. Then, the problem is to compute the interval availability complementary distribution

$$
\operatorname{IAVCD}(t, p)=P[\operatorname{IAV}(t)>p]
$$

where $0<p<1$. We will use the notation $S^{\prime}=S-\{r\}, U_{S}=U \cup S, D_{S}=D \cup S, U_{S}^{\prime}=U_{S}-\{r\}$, and $D_{S}^{\prime}=D_{S}-\{r\}$. In addition, we will let $\alpha_{i}=P[X(0)=i]$ and, being $B \subset \Omega, \alpha_{B}=\sum_{i \in B} \alpha_{i}$. Technically, to simplify the discussion, we will also assume that, being $\lambda_{i}$ the output rate of $X$ from state $i$, (C4) $\max _{i \in U} \lambda_{i}>0$ and $\max _{i \in D} \lambda_{i}>0$, (C5) if $U_{S}^{\prime} \neq \emptyset, X$ has some transition rate from $r$ to $U_{S}^{\prime}$, and (C6) if $U_{S}^{\prime} \neq \emptyset, \alpha_{D_{S}^{\prime}}>0$ and $\alpha_{U_{S}^{\prime}}=0, X$ has some transition rate from some state $i \in D_{S}^{\prime}$ with $\alpha_{i}>0$ to $U_{S}^{\prime}$. When $\max _{i \in D} \lambda_{i}=0$, all down states are absorbing and $\operatorname{IAVCD}(t, p)=P[X(p t) \in U]$. Similarly, in the case $\max _{i \in U} \lambda_{i}=0$, we have $\operatorname{IAVCD}(t, p)=$ $P[X((1-p) t) \in U]$. Therefore, when condition $\mathrm{C} 4$ is not satisfied, computation of $\operatorname{IAVCD}(t, p)$ can be reduced to a simpler problem. Condition $\mathrm{C} 5$ can be easily circumvented in practice by adding, in case $U_{S}^{\prime} \neq \emptyset$ and $X$ does not have any transition from $r$ to $U_{S}^{\prime}$, a tiny transition rate $\lambda \leqslant 10^{-10} \varepsilon /\left(2 t_{\max }\right)$ from $r$ to some state in $U_{S}^{\prime}$, where $\varepsilon$ is the allowed computation error and $t_{\text {max }}$ is the largest time $t$ at which $\operatorname{IAVCD}(t, p)$ has to be computed, with a negligible impact on $\operatorname{IAVCD}(t, p)$ no greater than $10^{-10} \varepsilon$ for $t \leqslant t_{\max }$ (see Lemma A1 in Appendix A) and small impact on the performance of the method. Condition C6 can be circumvented in practice in a similar way. The option $\Omega=S \cup\{f\}$ is allowed to cover bounding models [10], which are useful when an "exact" model has unmanageable size. In a lower bounding model, $S$ would be a subset of the states of the exact, complete model, $X$ will enter $f$ when the exact model exits $S$, the initial probability of $f$ would be the probability that initially the exact model is outside $S$, the subset of up states of the lower bounding model will include the up states in $S$ of the exact model, and $\operatorname{IAVCD}(t, p)$ would lower bound the interval availability complementary distribution of the exact model. In an upper bounding model, $S$ would be a subset of the states of the exact model, $X$ will enter $f$ when

\footnotetext{
${ }^{2}$ Given a CTMC with finite state space $\Omega$ and a potential regenerative state $r \in \Omega$, determination of whether conditions $\mathrm{C} 1$ and $\mathrm{C} 3$ hold with either $S=\Omega$ or $S=\Omega-\{f\}, f$ being an absorbing state, and $r \in S$ can be done as follows. If $X$ has no absorbing state, then $S$ must be $\Omega$ and it is enough to check that $X$ has a single trapping component $C$ and $r \in C$; if $X$ has a single absorbing state $a$ and $r \neq a$, then conditions $\mathrm{C} 1$ and $\mathrm{C} 3$ can only be satisfied with $S=\Omega-\{a\}$, and it is enough to check that either all states in $\Omega-\{a\}$ are transient or $X$ has a single trapping component $C \subset \Omega-\{a\}$ and $r \in C$; if $X$ has a single absorbing state $a$ and $r=a, S$ must be $\Omega$ and it is enough to check that $\{a\}$ is the only trapping component of $X$; if $X$ has two absorbing states $a, b$ and $r$ is one of them, say $a$, then $S$ must be $\Omega-\{b\}$ and it is enough to check that $\{a\}$ is the only trapping component of $X$ in $S$; finally, if $X$ has two absorbing states none of which is $r$ or $X$ has more than two absorbing states, then conditions $\mathrm{C} 1$ and $\mathrm{C} 3$ cannot be satisfied for any selection of $S$.
} 
the exact model exits $S$, the initial probability of $f$ would be the probability that initially the exact model is outside $S$, the subset of up states of the upper bounding model will include the up states in $S$ of the exact model and $f$, and $\operatorname{IAVCD}(t, p)$ would upper bound the interval availability complementary distribution of the exact model.

The new method requires the selection of a regenerative state $r \in S$ and its performance depends on that selection. The basic idea of the new method is to obtain a truncated transformed model, of potentially smaller size than the original model, by characterizing with enough accuracy the behavior of the original model from $S^{\prime}$ up to state $r$ or, if existent, the absorbing state $f$, and from $r$ until next hit of $r$ or, if existent, the absorbing state $f$, while keeping track of the amount of time spent in $U_{S}$, and solve the truncated transformed model using a state-of-the-art method (algorithm A of [6]). The rest of the paper is organized as follows. Section 2 briefly reviews algorithm A of [6]. In Section 3 we develop and describe the new method. Section 4 proves the so-called benign behavior of the new method, which implies that, for large enough models and large enough time intervals, the method will be significantly faster than previous methods. Also, for a class of models, class $\mathrm{C}_{1}$, including typical failure/repair models of coherent fault-tolerant systems with exponential failure and repair time distributions and repair in every state with failed components, for which a natural selection for the regenerative state exists, we will obtain stronger theoretical results assessing the performance of the new method for that natural selection in terms of "visible" model characteristics. Those results can be used to anticipate when the new method can be expected to be competitive for class $\mathrm{C}_{1}$ models. Section 5 analyzes the performance of the method using a large class $C_{1}$ model, showing that the method can indeed be significantly faster than algorithm $\mathrm{A}$ of [6] and is able to deal with some large models and large time intervals in reasonable CPU times. Appendix A includes some proofs. In the following, given a (homogeneous) discrete-time Markov chain (DTMC) $Y=\left\{Y_{k} ; k=0,1,2, \ldots\right\}$, we will denote by $Y_{m: n} c$ the predicate which is true when $Y_{k}$ satisfies condition $c$ for all $k, m \leqslant k \leqslant n$ (by convention $Y_{m: n} c$ will be true for $\left.m>n\right)$, and by \#( $\left.Y_{m: n} c\right)$ the number of indices $k, m \leqslant k \leqslant n$, for which $Y_{k}$ satisfies condition $c$. Also, given a matrix $\mathbf{A}, \mathbf{A}_{B, C}$ will denote the submatrix of $\mathbf{A}$ including the elements with subindices in $B \times C$, i.e. $\mathbf{A}_{B, C}=\left(a_{i, j}\right)_{i \in B, j \in C}$, and, given a vector $\mathbf{x}, \mathbf{x}^{B}$ will denote the subvector of $\mathbf{x}$ including the elements with subindices in $B$.

\section{Review of Algorithm A of [6]}

Algorithm A of [6], as most of the currently proposed methods to compute the interval availability distribution, is based on the randomization result. The method is applicable to any CTMC model $X$ with finite state space, $\Omega$. Let $\lambda_{i, j}, i, j \in \Omega, j \neq i$ denote the transition rate of $X$ from state $i$ to state $j$, and let $\lambda_{i}=\sum_{j \in \Omega-\{i\}} \lambda_{i, j}, i \in \Omega$ denote the output rate of $X$ of state $i$. Consider any $\Lambda \geqslant \max _{i \in \Omega} \lambda_{i}$ and define the DTMC $\hat{X}=\left\{\hat{X}_{k} ; k=0,1,2, \ldots\right\}$ with same state space and initial probability distribution as $X$ and transition probability matrix $\mathbf{P}=\left(P_{i, j}\right)_{i, j \in \Omega}$, where $P_{i, j}=\lambda_{i, j} / \Lambda, j \neq i$ and $P_{i, i}=1-\lambda_{i} / \Lambda$. Let $Q=\{Q(t) ; t \geqslant 0\}$ be a Poisson process with arrival rate $\Lambda\left(P[Q(t)=k]=\mathrm{e}^{-\Lambda t}(\Lambda t)^{k} / k !\right)$ independent of $\hat{X}$. Then, $X=\{X(t) ; t \geqslant 0\}$ is probabilistically identical to $\left\{\hat{X}_{Q(t)} ; t \geqslant 0\right\}$ (see, for instance, [11, Theorem 4.19]). The DTMC $\hat{X}$ is said to be the randomized DTMC of $X$ with randomization rate $\Lambda$; the CTMC $X$ is said to be the derandomized CTMC of $\hat{X}$ with randomization rate $\Lambda$. Using that result, we can interpret $X$ as the composition of the Poisson process $Q$ and the 
DTMC $\hat{X}$, in the sense that $X(t)$ is the state of $\hat{X}$ at the step given by the state in which $Q$ is at time $t$. Said in another way, we can see $X$ as the result of associating with the state visiting process $\hat{X}$ visits durations independent among them and independent of the path followed by $\hat{X}$ with exponential distributions with parameter $\Lambda$.

Let $U$ and $D$ denote, respectively, the sets of up and down states, and let $Y_{n, k}=P\left[\#\left(\hat{X}_{0: n} \in U>k\right)\right]$. Algorithm $\mathrm{A}$ of [6] is based on the following formulation for $\operatorname{IAVCD}(t, p)$ :

$$
\operatorname{IAVCD}(t, p)=\sum_{n=0}^{\infty} \mathrm{e}^{-\Lambda t} \frac{(\Lambda t)^{k}}{k !} \sum_{k=0}^{n}\left(\begin{array}{l}
n \\
k
\end{array}\right) p^{k} q^{n-k} Y_{n, k},
$$

where $q=1-p$. In the method, three truncations are performed on the summations of (1). Being $\varepsilon$ an error control parameter, the truncations are defined by the parameters:

$$
\begin{aligned}
& N=\min \left\{n \geqslant 0: \sum_{k=n+1}^{\infty} \mathrm{e}^{-\Lambda t} \frac{(\Lambda t)^{k}}{k !} \leqslant \frac{\varepsilon}{2}\right\}, \\
& C^{\prime \prime}= \begin{cases}\max \left\{c: 0 \leqslant c \leqslant N \wedge \sum_{k=0}^{c} \mathrm{e}^{-\Lambda t q} \frac{(\Lambda t q)^{k}}{k !} \leqslant \frac{\varepsilon}{4}\right\} & \text { if } \mathrm{e}^{-\Lambda t q} \leqslant \frac{\varepsilon}{4} \\
-1 & \text { if } \mathrm{e}^{-\Lambda t q}>\frac{\varepsilon}{4},\end{cases} \\
& C^{\prime}= \begin{cases}\min \left\{N, \min \left\{c \geqslant 0: \sum_{k=c+1}^{\infty} \mathrm{e}^{-\Lambda t q} \frac{(\Lambda t q)^{k}}{k !} \leqslant \frac{\varepsilon}{4}\right\}\right\} & \text { if } C^{\prime \prime} \neq-1 \\
\min \left\{c \geqslant 0: \sum_{k=c+1}^{\infty} \mathrm{e}^{-\Lambda t q} \frac{(\Lambda t q)^{k}}{k !} \leqslant \frac{\varepsilon}{2}\right\} & \text { if } C^{\prime \prime}=-1 .\end{cases}
\end{aligned}
$$

This gives the approximated value for $\operatorname{IAVCD}(t, p)$ :

$$
\operatorname{IAVCD}_{N, C^{\prime}, C^{\prime \prime}}^{a}(t, p)=\sum_{n=0}^{N} \mathrm{e}^{-\Lambda t} \frac{(\Lambda t)^{n}}{n !} \sum_{k=\max \left\{0, n-C^{\prime}\right\}}^{\min \left\{n, N-C^{\prime \prime}-1\right\}}\left(\begin{array}{l}
n \\
k
\end{array}\right) p^{k} q^{n-k} Y_{n, k},
$$

which satisfies

$$
\operatorname{IAVCD}(t, p)=\operatorname{IAVCD}_{N, C^{\prime}, C^{\prime \prime}}^{a}(t, p)+e_{N, C^{\prime}, C^{\prime \prime}}(t, p),
$$

with $0 \leqslant e_{N, C^{\prime}, C^{\prime \prime}}(t, p) \leqslant \varepsilon$.

Let $Y_{n, k}^{i}, i \in \Omega$ be the probability that a version of $\hat{X}, \hat{X}^{i}$, with initial state $i$ has performed more than $k$ visits to $U$ up to its $n$th transition, i.e. $Y_{n, k}^{i}=P\left[\#\left(\hat{X}_{0: n}^{i} \in U\right)>k\right]$, let $\mathbf{Y}_{n, k}$ be the column vector $\left(Y_{n, k}^{i}\right)_{i \in \Omega}$, and let $\boldsymbol{\alpha}$ be the initial probability distribution row vector of $X\left(\boldsymbol{\alpha}=\left(\alpha_{i}\right)_{i \in \Omega}, \alpha_{i}=P[X(0)=i]\right)$. We clearly have $Y_{n, k}=\alpha \mathbf{Y}_{n, k}$. Denoting by $\mathbf{0}$ and $\mathbf{1}$ column vectors of appropriate dimensions with all elements equal to, respectively, 0 and 1, the vectors $\mathbf{Y}_{n, k}$ in the domain of $(n, k)$ pairs for which $Y_{n, k}$ have to be obtained to compute $\operatorname{IAVCD}_{N, C^{\prime}, C^{\prime \prime}}^{a}(t, p)$ (illustrated in Fig. 1 for the case $0 \leqslant C^{\prime \prime}<C^{\prime}$ ) 


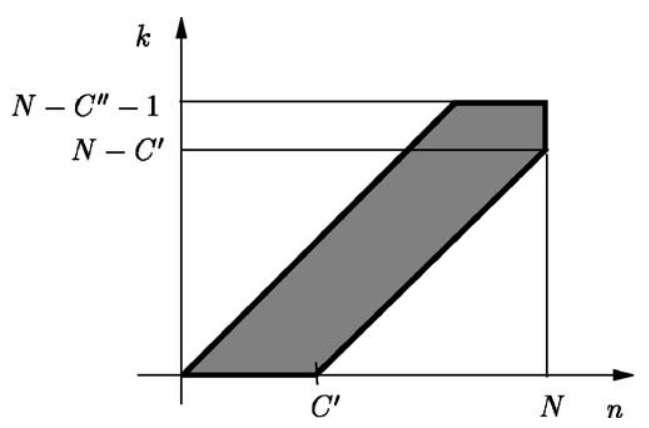

Fig. 1. Domain of $(n, k)$ pairs for which $Y_{n, k}$ has to be computed in Algorithm A of [6] for the case $0 \leqslant C^{\prime \prime}<C^{\prime}($ the domain includes the points in the frontier).

can be computed column-wise using the recurrences

$$
\begin{aligned}
& \mathbf{Y}_{n, k}^{U}=\mathbf{P}_{U, \Omega} \mathbf{Y}_{n-1, k-1}, \quad n>0,0<k \leqslant n, \\
& \mathbf{Y}_{n, 0}^{U}=\mathbf{1}, \quad n>0, \\
& \mathbf{Y}_{n, k}^{D}=\mathbf{P}_{D, \Omega} \mathbf{Y}_{n-1, k}, \quad n>0,0 \leqslant k<n, \\
& \mathbf{Y}_{n, n}^{D}=\mathbf{0}, \quad n>0
\end{aligned}
$$

with initial conditions

$$
\begin{aligned}
& \mathbf{Y}_{0,0}^{U}=\mathbf{1}, \\
& \mathbf{Y}_{0,0}^{D}=\mathbf{0} .
\end{aligned}
$$

The computational cost of the method is mainly determined by the truncation parameters $N$ and $C^{\prime}$, which increase with $\Lambda$, implying that the computational cost of the method will roughly increase with $\Lambda$ and, therefore, that $\Lambda$ should be taken equal to $\max _{i \in \Omega} \lambda_{i}$. For $N \gg 1, C^{\prime}, C^{\prime \prime} \ll N$ and not too small models, the flop count of the method is approximately equal to $2 N C^{\prime} T$, where $T$ is the number of transitions of $\hat{X}$. Using the well-known result [3, Theorem 3.3.5] that $Q(t)$ has for $\Lambda t \rightarrow \infty$ an asymptotic normal distribution with mean and variance $\Lambda t$, it is easy to realize that for large $\Lambda t$ and $\varepsilon \ll 1$ the required $N$ will be $\approx \Lambda t$ and, then, the method will be very costly for large models and large time intervals. The other proposed methods able to deal with general finite CTMCs $[2,5,9]$ have similar problems.

\section{The method}

\subsection{Preliminaries}

As in the previous section, we will denote by $\lambda_{i, j}$ the transition rates of $X$ and by $\lambda_{i}$ the output rates of $X$. Also, being $B \subset \Omega-\{i\}$, we will denote by $\lambda_{i, B}$ the transition rate from state $i$ to subset $B$, i.e. $\lambda_{i, B}=\sum_{j \in B} \lambda_{i, j}$. 
We will consider a DTMC $\hat{X}$ obtained from $X$ by randomizing $X$ with rate $\Lambda_{U} \geqslant \max _{i \in U} \lambda_{i}$ in the states $i \in U$ and with rate $\Lambda_{D} \geqslant \max _{i \in D} \lambda_{i}$ in the states $i \in D$, where $\Lambda_{U}$ and $\Lambda_{D}$ can, in general, be different. The DTMC $\hat{X}$ has the same state space and initial probability distribution as $X$ and transition probabilities $P_{i, j}=\lambda_{i, j} / \Lambda_{U}, i \in U, j \neq i, P_{i, i}=1-\lambda_{i} / \Lambda_{U}, i \in U, P_{i, j}=\lambda_{i, j} / \Lambda_{D}, i \in D, j \neq i$, and $P_{i, i}=1-\lambda_{i} / \Lambda_{D}, i \in D$. Being $B \subset \Omega$, we will use the notation $P_{i, B}=\sum_{j \in B} P_{i, j}$. Let $\mathbf{P}=\left(P_{i, j}\right)_{i, j \in \Omega}$ denote the transition probability matrix of $\hat{X}$. We can interpret $X$ as the result of associating with the state visiting process $\hat{X}$ visit durations independent among them and independent of the path followed by $\hat{X}$ with exponential distributions with parameter $\Lambda_{U}$ in the states $i \in U$ and parameter $\Lambda_{D}$ in the states $i \in D$. The correctness of that interpretation can be easily shown by noting that states in $\hat{X}$ are absorbing if and only if they are absorbing in $X$ and, for non-absorbing states $i$, computing, using the interpretation, the Markov kernel [12] of the resulting semi-Markov process and checking that the kernel probabilities have values $Q_{i, j}(t)=\lambda_{i, j} / \lambda_{i}\left(1-\mathrm{e}^{-\lambda_{i} t}\right)$, which coincide with the kernel probabilities of $X$, seen as a semi-Markov process. We will say that $\hat{X}$ is the randomized DTMC of $X$ with randomization rate $\Lambda_{U}$ in $U$ and $\Lambda_{D}$ in $D ; X$ will be said to be the derandomized CTMC of $\hat{X}$ with randomization rate $\Lambda_{U}$ in $U$ and $\Lambda_{D}$ in $D$.

To simplify the discussion, we will assume that $\Lambda_{U}$ and $\Lambda_{D}$ are taken slightly larger than, respectively, $\max _{i \in U} \lambda_{i}$ and $\max _{i \in D} \lambda_{i}$, i.e. $\Lambda_{U}=(1+\theta) \max _{i \in U} \lambda_{i}>0$ and $\Lambda_{D}=(1+\theta) \max _{i \in D} \lambda_{i}>0$, where $\theta$ is a small positive quantity, say $\theta=10^{-4}$. This implies $P_{i, i}>0, i \in \Omega$.

\subsection{The transformation}

In this section we will transform $X$ into a (homogeneous) CTMC $V$ with same interval availability distribution as $X$. The CTMC $V$ has, in most cases, an infinite state space; however, it will be shown in the next section how its state space can be truncated to obtain a CTMC with finite state space which has with some arbitrarily small error the same interval availability distribution as $X$. The transformation follows ideas similar to those used in the regenerative randomization method $[13,14]$. The basic idea to perform the transformation is to characterize the behavior of $X$ from $S^{\prime}$ up to state $r$ or, if existent, the absorbing state $f$, and from $r$ until next hit of $r$ or, if existent, the absorbing state $f$, while keeping track of the amount of time spent in $U_{S}$. To avoid excessive discussion, we will only consider explicitly the case $\Omega=S \cup\{f\}$. The developments carry out immediately to the case $\Omega=S$ by eliminating the state $f$ from definitions and sets. To construct the transformed model $V$, two (homogeneous) DTMCs $Z, Z^{\prime}$ will have to be analyzed, in general.

The DTMC $Z=\left\{Z_{n} ; n=0,1,2, \ldots\right\}$ follows $\hat{X}$ from $r$ till re-entry in $r$. Formally, considering a version of $\hat{X}, \hat{X}^{\prime}$, with initial probability distribution concentrated in state $r$,

$$
\begin{aligned}
& Z_{0}=r, \\
& Z_{n}=\left\{\begin{array}{ll}
i \in S^{\prime} \cup\{f\} & \text { if } \hat{X}_{1: n}^{\prime} \neq r \wedge \hat{X}_{n}^{\prime}=i, \\
a & \text { if } \#\left(\hat{X}_{1: n}^{\prime}=r\right)>0,
\end{array} n>0 .\right.
\end{aligned}
$$

The DTMC $Z$ has state space $S \cup\{f, a\}$, where $f$ and $a$ are absorbing states and, given the assumed properties for $X$, all states in $S$ are transient, and its (possibly) non-null transition probabilities 
are

$$
\begin{aligned}
& P\left[Z_{n+1}=j \mid Z_{n}=i\right]=P_{i, j}, \quad i \in S, j \in S^{\prime} \cup\{f\}, \\
& P\left[Z_{n+1}=a \mid Z_{n}=i\right]=P_{i, r}, \quad i \in S, \\
& P\left[Z_{n+1}=f \mid Z_{n}=f\right]=P\left[Z_{n+1}=a \mid Z_{n}=a\right]=1 .
\end{aligned}
$$

The second DTMC, $Z^{\prime}=\left\{Z_{n}^{\prime} ; n=0,1,2, \ldots\right\}$, follows $\hat{X}$ until its first visit to state $r$. Formally, $Z^{\prime}$ is defined as

$$
Z_{n}^{\prime}= \begin{cases}i \in S^{\prime} \cup\{f\} & \text { if } \hat{X}_{0: n} \neq r \wedge \hat{X}_{n}=i, \\ a & \text { if } \#\left(\hat{X}_{0: n}=r\right)>0 .\end{cases}
$$

The DTMC $Z^{\prime}$ has state space $S^{\prime} \cup\{f, a\}$, where $f$ and $a$ are absorbing states and, given the assumed properties for $X$, all states in $S^{\prime}$ are transient. The initial probability distribution of $Z^{\prime}$ is $P\left[Z_{0}^{\prime}=i\right]=\alpha_{i}, i \in S^{\prime} \cup\{f\}, P\left[Z_{0}^{\prime}=a\right]=\alpha_{r}$, and its (possibly) non-null transition probabilities are

$$
\begin{aligned}
& P\left[Z_{n+1}^{\prime}=j \mid Z_{n}^{\prime}=i\right]=P_{i, j}, \quad i \in S^{\prime}, j \in S^{\prime} \cup\{f\}, \\
& P\left[Z_{n+1}^{\prime}=a \mid Z_{n}^{\prime}=i\right]=P_{i, r}, \quad i \in S^{\prime}, \\
& P\left[Z_{n+1}^{\prime}=f \mid Z_{n}^{\prime}=f\right]=P\left[Z_{n+1}^{\prime}=a \mid Z_{n}^{\prime}=a\right]=1 .
\end{aligned}
$$

Let $\pi_{i}(n, k)=P\left[Z_{n}=i \wedge \#\left(Z_{0: n} \in U_{S}\right)=k\right], i \in S$ and let $\pi_{i}^{\prime}(n, k)=P\left[Z_{n}^{\prime}=i \wedge \#\left(Z_{0: n}^{\prime} \in U_{S}^{\prime}\right)=k\right], i \in S^{\prime}$. Denoting by $\mathbf{0}$ and 1 row vectors of appropriate dimensions with all its elements equal to, respectively, 0 and 1 , the row vectors $\pi(n, k)=\left(\pi_{i}(n, k)\right)_{i \in S}, n \geqslant 0,0 \leqslant k \leqslant n+1$ can be obtained using the recurrences

$$
\begin{aligned}
& \pi(n, k)^{U_{S}^{\prime}}=\pi(n-1, k-1) \mathbf{P}_{S, U_{S}^{\prime}}, \quad n \geqslant 1,1 \leqslant k \leqslant n+1, \\
& \pi(n, k)^{D_{S}^{\prime}}=\pi(n-1, k) \mathbf{P}_{S, D_{S}^{\prime}}, \quad n \geqslant 1,0 \leqslant k \leqslant n
\end{aligned}
$$

and

$$
\begin{aligned}
& \pi_{r}(n, k)=0, \quad n \geqslant 1,0 \leqslant k \leqslant n+1, \\
& \pi_{r}(0,0)=I_{r \in D_{S}}, \\
& \pi_{r}(0,1)=I_{r \in U_{S}}, \\
& \pi(0, k)^{U_{S}^{\prime}}=\mathbf{0}, \quad 0 \leqslant k \leqslant 1, \\
& \pi(n, 0)^{U_{S}^{\prime}}=\mathbf{0}, \quad n \geqslant 1,
\end{aligned}
$$




$$
\begin{aligned}
& \pi(0, k)^{D_{S}^{\prime}}=\mathbf{0}, \quad 0 \leqslant k \leqslant 1, \\
& \pi(n, n+1)^{D_{S}^{\prime}}=\mathbf{0}, \quad n \geqslant 1 .
\end{aligned}
$$

Similarly, the row vectors $\pi^{\prime}(n, k)=\left(\pi_{i}^{\prime}(n, k)\right)_{i \in S^{\prime}}, n \geqslant 0,0 \leqslant k \leqslant n+1$ can be obtained using the recurrences

$$
\begin{aligned}
& \pi^{\prime}(n, k)^{U_{S}^{\prime}}=\pi^{\prime}(n-1, k-1) \mathbf{P}_{S^{\prime}, U_{S}^{\prime}}, \quad n \geqslant 1,1 \leqslant k \leqslant n+1, \\
& \pi^{\prime}(n, k)^{D_{S}^{\prime}}=\pi^{\prime}(n-1, k) \mathbf{P}_{S^{\prime}, D_{S}^{\prime}}, \quad n \geqslant 1,0 \leqslant k \leqslant n
\end{aligned}
$$

and

$$
\begin{aligned}
& \pi^{\prime}(0,0)^{U_{S}^{\prime}}=\mathbf{0}, \\
& \boldsymbol{\pi}^{\prime}(0,1)^{U_{S}^{\prime}}=\boldsymbol{\alpha}^{U_{S}^{\prime}}, \\
& \boldsymbol{\pi}^{\prime}(n, 0)^{U_{S}^{\prime}}=\mathbf{0}, \quad n \geqslant 1, \\
& \boldsymbol{\pi}^{\prime}(0,0)^{D_{S}^{\prime}}=\boldsymbol{\alpha}^{D_{S}^{\prime}}, \\
& \pi^{\prime}(0,1)^{D_{S}^{\prime}}=\mathbf{0}, \\
& \pi^{\prime}(n, n+1)^{D_{S}^{\prime}}=\mathbf{0}, \quad n \geqslant 1 .
\end{aligned}
$$

To derive the transformed CTMC $V$, we will consider the discrete-time stochastic process $\tilde{V}=\left\{\tilde{V}_{n} ; n=0,1,2, \ldots\right\}$ defined from $\hat{X}$ as follows:

$$
\tilde{V}_{n}= \begin{cases}s_{l, k}^{u} & \text { if } 0 \leqslant l \leqslant n \wedge \hat{X}_{n-l}=r \wedge \hat{X}_{n-l+1: n} \in S^{\prime} \wedge \#\left(\hat{X}_{n-l: n} \in U_{S}\right)=k \wedge \hat{X}_{n} \in U_{S}, \\ s_{l, k}^{d} & \text { if } 0 \leqslant l \leqslant n \wedge \hat{X}_{n-l}=r \wedge \hat{X}_{n-l+1: n} \in S^{\prime} \wedge \#\left(\hat{X}_{n-l: n} \in U_{S}\right)=k \wedge \hat{X}_{n} \in D_{S}, \\ s_{n, k}^{\prime \prime} & \text { if } \hat{X}_{0: n} \in S^{\prime} \wedge \#\left(\hat{X}_{0: n} \in U_{S}^{\prime}\right)=k \wedge \hat{X}_{n} \in U_{S}^{\prime}, \\ s_{n, k}^{\prime d} & \text { if } \hat{X}_{0: n} \in S^{\prime} \wedge \#\left(\hat{X}_{0: n} \in U_{S}^{\prime}\right)=k \wedge \hat{X}_{n} \in D_{S}^{\prime}, \\ f & \text { if } \hat{X}_{n}=f .\end{cases}
$$

In words, $\tilde{V}_{n}=s_{l, k}^{u}$ if, by step $n, \hat{X}$ has not left $S$, has made some visit to $r$, the last visit to $r$ was $l$ steps before, $k$ of the visits since then have been to states in $U_{S}$, and the current state is in $U_{S}$; $\tilde{V}_{n}=s_{l, k}^{d}$ if, by step $n, \hat{X}$ has not left $S$, has made some visit to $r$, the last visit to $r$ was $l$ steps before, $k$ of the visits since then have been to states in $U_{S}$, and the current state is in $D_{S} ; \tilde{V}_{n}=s_{n, k}^{\prime u}$ if, by step $n, \hat{X}$ has not left $S^{\prime}, k$ visits have been to states in $U_{S}^{\prime}\left(U_{S}\right)$ and the current state is in $U_{S}^{\prime}\left(U_{S}\right) ; \tilde{V}_{n}=s_{n, k}^{\prime d}$ if, by step $n, \hat{X}$ has not left $S^{\prime}, k$ visits have been to states in $U_{S}^{\prime}\left(U_{S}\right)$ and the current state is in $D_{S}^{\prime}\left(D_{S}\right)$; and $\tilde{V}_{n}=f$ if, by step $n, \hat{X}$ has been absorbed into state $f$. Note that, 
if $r \in U_{S}, \tilde{V}_{n}=s_{0,1}^{u}$ if and only if $\hat{X}_{n}=r$ and, if $r \in D_{S}, \tilde{V}_{n}=s_{0,0}^{d}$ if and only if $\hat{X}_{n}=r$. To simplify the notation, let $s_{0,}^{\cdot}$. denote state $s_{0,1}^{u}$ if $r \in U_{S}$ and state $s_{0,0}^{d}$ if $r \in D_{S}$. Since $\hat{X}$ is a DTMC and: (1) $\tilde{V}_{n}=s_{0 \text {, }}$ if and only if $\hat{X}_{n}=r$, (2) $\tilde{V}_{n}$ only depends on $\hat{X}_{0}, \hat{X}_{1}, \ldots, \hat{X}_{n}$, and (3) assuming $\hat{X}_{m}=r, \tilde{V}_{m+n}$ does not depend on $\hat{X}_{0}, \ldots, \hat{X}_{m-1}$ and depends on $\hat{X}_{m+1}, \ldots, \hat{X}_{m+n}$ as, assuming $\hat{X}_{0}=r, \tilde{V}_{n}$ depends on $\hat{X}_{1}, \ldots, \hat{X}_{n}$, the steps at which $\tilde{V}$ hits state $s_{0, \text {. }}$ are regeneration points of $\tilde{V}$. It follows from its definition that the state space of $\tilde{V}$ is ${ }^{V} \Omega=\left\{s_{n, k}^{u}: \pi(n, k)^{U_{S}} \neq\right.$ $\mathbf{0}\} \cup\left\{s_{n, k}^{d}: \pi(n, k)^{D_{S}} \neq \mathbf{0}\right\} \cup\left\{s_{n, k}^{\prime \prime}: \pi^{\prime}(n, k)^{U_{S}^{\prime}} \neq \mathbf{0}\right\} \cup\left\{s_{n, k}^{\prime d}: \pi^{\prime}(n, k)^{D_{S}^{\prime}} \neq \mathbf{0}\right\} \cup\{f\}^{3}$ and its initial probability distribution is

$$
\begin{aligned}
& P\left[\tilde{V}_{0}=s_{0, .}\right]=\alpha_{r}, \\
& P\left[\tilde{V}_{0}=s_{0,1}^{\prime u}\right]=\alpha_{U_{S}^{\prime}}, \\
& P\left[\tilde{V}_{0}=s_{0,0}^{\prime d}\right]=\alpha_{D_{S}^{\prime}}, \\
& P\left[\tilde{V}_{0}=f\right]=\alpha_{f}, \\
& P\left[\tilde{V}_{0}=i\right]=0, \quad i \notin\left\{\dot{s_{0,}}, s_{0,1}^{\prime u}, s_{0,0}^{\prime d}, f\right\} .
\end{aligned}
$$

It is also clear that: (1) $\tilde{V}_{n} \in\left\{s_{l, k}^{u}, 0 \leqslant l \leqslant n, 1 \leqslant k \leqslant n+1\right\} \cup\left\{s_{l, k}^{d}, 0 \leqslant l \leqslant n, 0 \leqslant k \leqslant n\right\} \cup\left\{s_{n, k}^{\prime u}, 1 \leqslant k\right.$ $\leqslant n+1\} \cup\left\{s_{n, k}^{\prime d}, 0 \leqslant k \leqslant n\right\} \cup\{f\}$, (2) $\tilde{V}_{n}=f$ implies $\tilde{V}_{n+1}=f$, (3) both $\tilde{V}_{n}=s_{l, k}^{u}$ and $\tilde{V}_{n}=s_{l, k}^{d}$ imply $\tilde{V}_{n+1} \in\left\{s_{0, .}^{\prime}, s_{l+1, k+1}^{u}, s_{l+1, k}^{d}, f\right\}$, and (4) both $\tilde{V}_{n}=s_{n, k}^{\prime \prime}$ and $\tilde{V}_{n}=s_{n, k}^{\prime d}$ imply $\tilde{V}_{n+1} \in\left\{s_{0, .}^{\cdot}, s_{n+1, k+1}^{\prime \prime}\right.$, $\left.s_{n+1, k}^{\prime d}, f\right\}$.

The discrete-time stochastic process $\tilde{V}$ is not, in general, a DTMC. It satisfies, however, an important property: that all non-null probabilities $P\left[\tilde{V}_{n+1}=y \mid \tilde{V}_{n}=x\right]$ which are well-defined, i.e. such that $P\left[\tilde{V}_{n}=x\right]>0$, only depend on $x$ and $y$ and are independent of $n$. This is established by the following proposition.

Proposition 1. Let $v_{l, k}^{u}=\left(\sum_{i \in U_{S}} \pi_{i}(l, k) P_{i, f}\right) / \sum_{i \in U_{S}} \pi_{i}(l, k), \quad v_{l, k}^{d}=\left(\sum_{i \in D_{S}} \pi_{i}(l, k) P_{i, f}\right) /$ $\sum_{i \in D_{S}} \pi_{i}(l, k), q_{l, k}^{u}=\left(\sum_{i \in U_{S}} \pi_{i}(l, k) P_{i, r}\right) / \sum_{i \in U_{S}} \pi_{i}(l, k), q_{l, k}^{d}=\left(\sum_{i \in D_{S}} \pi_{i}(l, k) P_{i, r}\right) / \sum_{i \in D_{S}} \pi_{i}(l, k)$, $w_{l, k}^{u u}=\left(\sum_{i \in U_{S}} \pi_{i}(l, k) P_{i, U_{S}^{\prime}}\right) / \sum_{i \in U_{S}} \pi_{i}(l, k), \quad w_{l, k}^{u d}=\left(\sum_{i \in U_{S}} \pi_{i}(l, k) P_{i, D_{S}^{\prime}}\right) / \sum_{i \in U_{S}} \pi_{i}(l, k), \quad w_{l, k}^{d u}=$ $\left(\sum_{i \in D_{S}} \pi_{i}(l, k) P_{i, U_{S}^{\prime}}\right) / \sum_{i \in D_{S}} \pi_{i}(l, k), w_{l, k}^{d d}=\left(\sum_{i \in D_{S}} \pi_{i}(l, k) P_{i, D_{S}^{\prime}}\right) / \sum_{i \in D_{S}} \pi_{i}(l, k), v_{l, k}^{\prime u}=$

\footnotetext{
${ }^{3}$ We only include in ${ }^{V} \Omega$ reachable states. That $f$ is reachable follows from $f$ being reachable in $\hat{X}$ and $\tilde{V}_{n}=f$ if and only if $\hat{X}_{n}=f$. That $s_{0,}$. is reachable follows from $r$ being reachable in $\hat{X}$ and $\tilde{V}_{n}=s_{0,}$. if and only if $\hat{X}_{n}=r$. Both $\tilde{V}_{m}=s_{n, k}^{u}$ and $\tilde{V}_{m}=s_{n, k}^{d}$ imply $\tilde{V}_{m-n}=s_{0, .}$. Using the $\tilde{w}^{u}(n, k)$ and $\tilde{w}^{d}(n, k)$ defined in the proof of Proposition 2, $P\left[\tilde{V}_{m}=s_{n, k}^{u}\right]=\tilde{w}^{u}(n, k) P\left[\tilde{V}_{m-n}=\dot{s_{0,}}\right]$ and $P\left[\tilde{V}_{m}=s_{n, k}^{d}\right]=\tilde{w}^{d}(n, k) P\left[\tilde{V}_{m-n}=\dot{s_{0,}}\right.$. $]$. This implies that are reachable exactly the states $s_{n, k}^{u}$ with $\tilde{w}^{u}(n, k) \neq 0$ and the states $s_{n, k}^{d}$ with $\tilde{w}^{d}(n, k) \neq 0$. But, it is shown in the proof of Proposition 2 that $\tilde{w}^{u}(n, k)=\sum_{i \in U_{S}} \pi_{i}(n, k)$ and $\tilde{w}^{d}(n, k)=\sum_{i \in D_{S}} \pi_{i}(n, k)$ and, therefore, are reachable exactly the states $s_{n, k}^{u}$ with $\pi(n, k)^{U_{S}} \neq \mathbf{0}$ and the states $s_{n, k}^{d}$ with $\pi(n, k)^{D_{S}} \neq \mathbf{0}$. Finally, $\tilde{V}$ can only be at a state $s_{n, k}^{\prime \prime}$ or $s_{n, k}^{\prime d}$ at step $n$. Using the $\tilde{w}^{\prime \prime}(n, k)$ and $\tilde{w}^{\prime d}(n, k)$ defined in the proof of Proposition 2, $P\left[\tilde{V}_{n}=s_{n, k}^{\prime \prime}\right]=\tilde{w}^{\prime \prime}(n, k)$ and $P\left[\tilde{V}_{n}=s_{n, k}^{\prime d}\right]=\tilde{w}^{\prime d}(n, k)$. But, it is shown in the proof of Proposition 2 that $\tilde{w}^{\prime \prime}(n, k)=\sum_{i \in U_{S}^{\prime}} \pi_{i}^{\prime}(n, k)$ and $\tilde{w}^{\prime d}(n, k)=\sum_{i \in D_{S}^{\prime}} \pi_{i}^{\prime}(n, k)$ and, therefore, are reachable exactly the states $s_{n, k}^{\prime \prime}$ with $\pi^{\prime}(n, k)^{U_{S}^{\prime}} \neq \mathbf{0}$ and the states $s_{n, k}^{\prime d}$ with $\pi^{\prime}(n, k)^{D_{S}^{\prime}} \neq \mathbf{0}$.
} 
$\left(\sum_{i \in U_{S}^{\prime}} \pi_{i}^{\prime}(l, k) P_{i, f}\right) / \sum_{i \in U_{S}^{\prime}} \pi_{i}^{\prime}(l, k), \quad v_{l, k}^{\prime d}=\left(\sum_{i \in D_{S}^{\prime}} \pi_{i}^{\prime}(l, k) P_{i, f}\right) / \sum_{i \in D_{S}^{\prime}} \pi_{i}^{\prime}(l, k), \quad q_{l, k}^{\prime u}=$ $\left(\sum_{i \in U_{S}^{\prime}} \pi_{i}^{\prime}(l, k) P_{i, r}\right) / \sum_{i \in U_{S}^{\prime}} \pi_{i}^{\prime}(l, k), \quad q_{l, k}^{\prime d}=\left(\sum_{i \in D_{S}^{\prime}} \pi_{i}^{\prime}(l, k) P_{i, r}\right) / \sum_{i \in D_{S}^{\prime}} \pi_{i}^{\prime}(l, k), \quad w_{l, k}^{\prime u u}=$ $\left(\sum_{i \in U_{S}^{\prime}} \pi_{i}^{\prime}(l, k) P_{i, U_{S}^{\prime}}\right) / \sum_{i \in U_{S}^{\prime}} \pi_{i}^{\prime}(l, k), \quad w_{l, k}^{\prime \prime d}=\left(\sum_{i \in U_{S}^{\prime}} \pi_{i}^{\prime}(l, k) P_{i, D_{S}^{\prime}}\right) / \sum_{i \in U_{S}^{\prime}} \pi_{i}^{\prime}(l, k), \quad w_{l, k}^{\prime d u}=$ $\left(\sum_{i \in D_{S}^{\prime}} \pi_{i}^{\prime}(l, k) P_{i, U_{S}^{\prime}}\right) / \sum_{i \in D_{S}^{\prime}} \pi_{i}^{\prime}(l, k)$, and $w_{l, k}^{\prime d d}=\left(\sum_{i \in D_{S}^{\prime}} \pi_{i}^{\prime}(l, k) P_{i, D_{S}^{\prime}}\right) / \sum_{i \in D_{S}^{\prime}} \pi_{i}^{\prime}(l, k)$. Then, the values of the well-defined, non-null probabilities $P\left[\tilde{V}_{n+1}=y \mid \tilde{V}_{n}=x\right]$ are: $P\left[\tilde{V}_{n+1}=f \mid \tilde{V}_{n}=s_{l, k}^{u}\right]=v_{l, k}^{u}$, $P\left[\tilde{V}_{n+1}=s_{0, .}^{\cdot} \mid \tilde{V}_{n}=s_{l, k}^{u}\right]=q_{l, k}^{u}, P\left[\tilde{V}_{n+1}=s_{l+1, k+1}^{u} \mid \tilde{V}_{n}=s_{l, k}^{u}\right]=w_{l, k}^{u u}, P\left[\tilde{V}_{n+1}=s_{l+1, k}^{d} \mid \tilde{V}_{n}=s_{l, k}^{u}\right]=w_{l, k}^{u d}$, $P\left[\tilde{V}_{n+1}=f \mid \tilde{V}_{n}=s_{l, k}^{d}\right]=v_{l, k}^{d}, \quad P\left[\tilde{V}_{n+1}=s_{0, .} \mid \tilde{V}_{n}=s_{l, k}^{d}\right]=q_{l, k}^{d}, \quad P\left[\tilde{V}_{n+1}=s_{l+1, k+1}^{u} \mid \tilde{V}_{n}=s_{l, k}^{d}\right]=w_{l, k}^{d u}$, $P\left[\tilde{V}_{n+1}=s_{l+1, k}^{d} \mid \tilde{V}_{n}=s_{l, k}^{d}\right]=w_{l, k}^{d d}, \quad P\left[\tilde{V}_{n+1}=f \mid \tilde{V}_{n}=s_{l, k}^{\prime u}\right]=v_{l, k}^{\prime u}, \quad P\left[\tilde{V}_{n+1}=s_{0, .} . \tilde{V}_{n}=s_{l, k}^{\prime \prime}\right]=q_{l, k}^{\prime u}$, $P\left[\tilde{V}_{n+1}=s_{l+1, k+1}^{\prime u} \mid \tilde{V}_{n}=s_{l, k}^{\prime u}\right]=w_{l, k}^{\prime u u}, \quad P\left[\tilde{V}_{n+1}=s_{l+1, k}^{\prime d} \mid \tilde{V}_{n}=s_{l, k}^{\prime u}\right]=w_{l, k}^{\prime u d}, \quad P\left[\tilde{V}_{n+1}=f \mid \tilde{V}_{n}=s_{l, k}^{\prime d}\right]=v_{l, k}^{\prime d}$, $P\left[\tilde{V}_{n+1}=s_{0, .}^{\prime} \mid \tilde{V}_{n}=s_{l, k}^{\prime d}\right]=q_{l, k}^{\prime d}, \quad P\left[\tilde{V}_{n+1}=s_{l+1, k+1}^{\prime u} \mid \tilde{V}_{n}=s_{l, k}^{\prime d}\right]=w_{l, k}^{\prime d u}, \quad P\left[\tilde{V}_{n+1}=s_{l+1, k}^{\prime d} \mid \tilde{V}_{n}=s_{l, k}^{\prime d}\right]=w_{l, k}^{\prime d d}$, and $P\left[\tilde{V}_{n+1}=f \mid \tilde{V}_{n}=f\right]=1$.

Proof. See the Appendix A.

Consider now the (homogeneous) DTMC $\hat{V}$ with same state space and initial probability distribution as $\tilde{V}$ and (assuming $P\left[\hat{V}_{n}=x\right]>0$ ) non-null transition probabilities $P\left[\hat{V}_{n+1}=y \mid \hat{V}_{n}=x\right]$ identical to the non-null, well-defined transition probabilities $P\left[\tilde{V}_{n+1}=y \mid \tilde{V}_{n}=x\right]$ given by Proposition 1 . Note that the definition is correct because the well-defined transition probabilities $P\left[\tilde{V}_{n+1}=y \mid \tilde{V}_{n}=x\right]$ do not depend on $n$ (did they depend on $n, \hat{V}$ would not be homogeneous). Fig. 2 depicts a feasible state transition diagram for $\hat{V}$ for the case $r \in U_{S}$. Let the state space ${ }^{V} \Omega$ of $\tilde{V}$ and $\hat{V}$ be partitioned as ${ }^{V} U \cup{ }^{V} D$, where ${ }^{V} U$ includes the states $s_{n, k}^{u}, s_{n, k}^{\prime u}$, and the state $f$ if $f \in U$, and ${ }^{V} D$ includes the states $s_{n, k}^{d}, s_{n, k}^{\prime d}$, and the state $f$ if $f \in D$. Note that $\tilde{V}_{n} \in{ }^{V} U$ if and only if $\hat{X}_{n} \in U$. Let ${ }^{V} S$ be the subset of ${ }^{V} \Omega$ including all states in ${ }^{V} \Omega$ except state $f$. Let ${ }^{V} S^{\prime}={ }^{V} S-\left\{s \cdot s_{0,}\right\},{ }^{V} U_{S}={ }^{V} U \cap{ }^{V} S$, ${ }^{V} D_{S}={ }^{V} D \cap{ }^{V} S,{ }^{V} U_{S}^{\prime}={ }^{V} U_{S}-\left\{s_{0,}^{\cdot}\right\}$, and ${ }^{V} D_{S}^{\prime}={ }^{V} D_{S}-\left\{s_{0,}.\right\}$. We have the following result:

Proposition 2. $P\left[\#\left(\hat{V}_{0: n} \in{ }^{V} U\right)=k \wedge \hat{V}_{n} \in{ }^{V} U\right]=P\left[\#\left(\tilde{V}_{0: n} \in{ }^{V} U\right)=k \wedge \tilde{V}_{n} \in{ }^{V} U\right], 1 \leqslant k \leqslant n+1$ and $P\left[\#\left(\hat{V}_{0: n} \in{ }^{V} U\right)=k \wedge \hat{V}_{n} \in{ }^{V} D\right]=P\left[\#\left(\tilde{V}_{0: n} \in{ }^{V} U\right)=k \wedge \tilde{V}_{n} \in{ }^{V} D\right], 0 \leqslant k \leqslant n$.

Proof. See the Appendix A.

The transformed CTMC $V$ is the derandomized CTMC of $\hat{V}$ with randomization rate $\Lambda_{U}$ in ${ }^{V} U$ and $\Lambda_{D}$ in ${ }^{V} D$. The state space and initial probability distribution of $V$ are the same as those of $\hat{V}$. Fig. 3 depicts a feasible state transition diagram of $V$ for the case $r \in U_{S}$. The following theorem establishes the main result of this section.

Theorem 1. The interval availability complementary distribution of $V$ with subset of up states ${ }^{V} U$ is equal to the interval availability complementary distribution of $X$.

Proof. Consider the interpretation of $X$ as the derandomized CTMC of $\hat{X}$ with randomization rate $\Lambda_{U}$ in $U$ and $\Lambda_{D}$ in $D$. Under that interpretation, let $X_{i}^{U}$ be the random variable "duration of the $i$ th visit of $\hat{X}$ to a state in $U$ " and let $X_{i}^{D}$ be the random variable "duration of the $i$ th visit of $\hat{X}$ to 


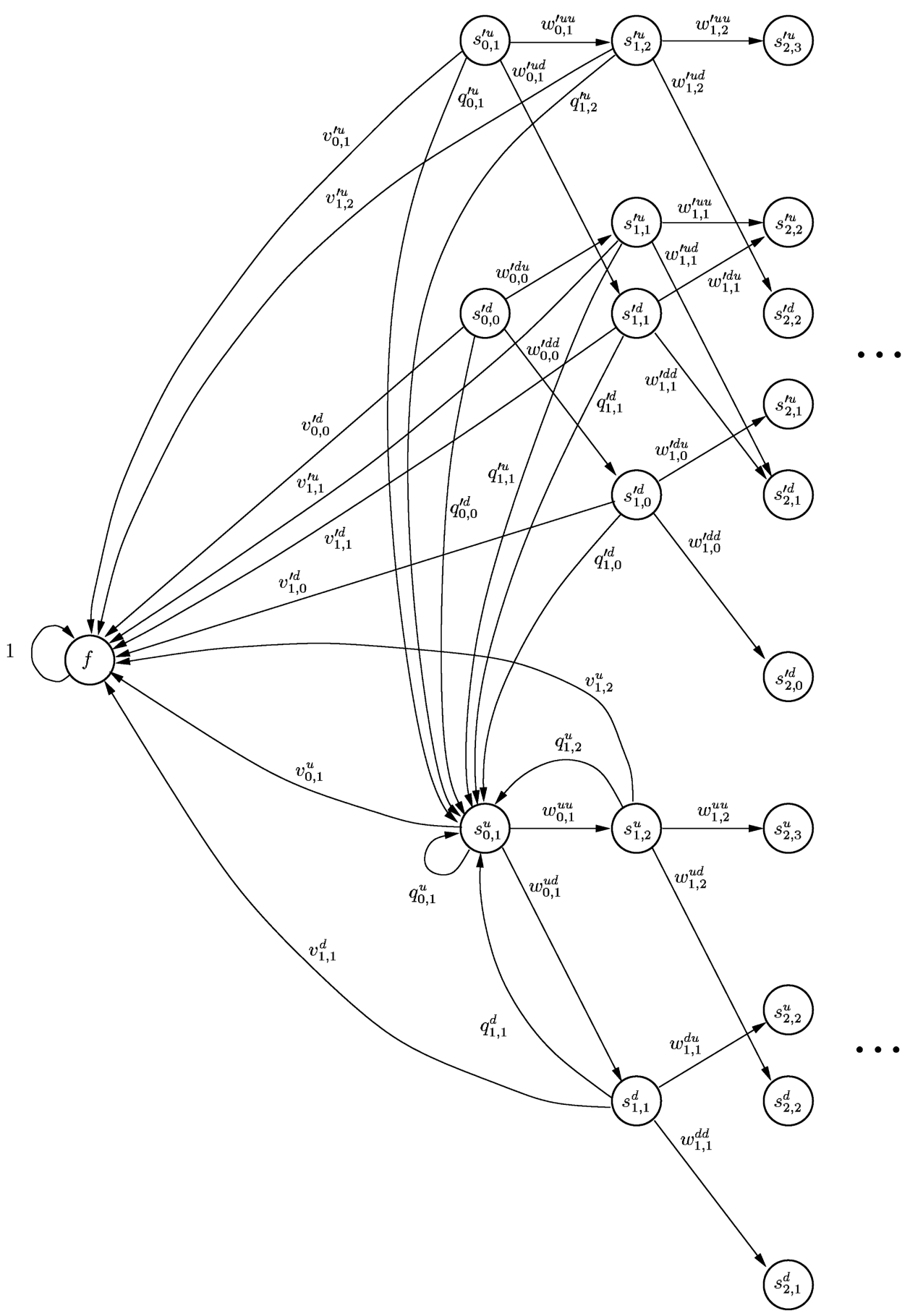

Fig. 2. A feasible state transition diagram of the DTMC $\hat{V}$ for the case $r \in U_{S}$. 


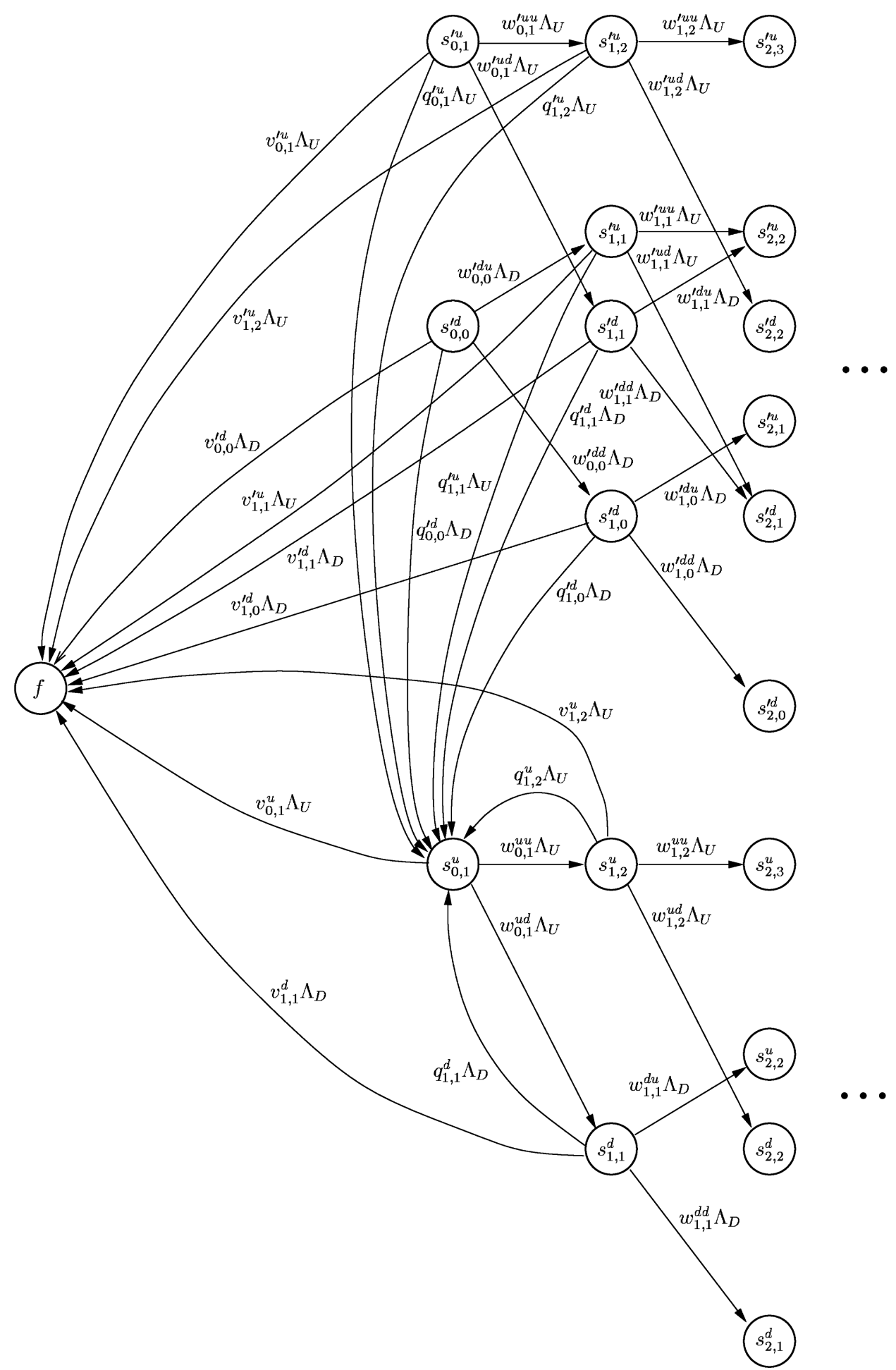

Fig. 3. A feasible state transition diagram of the CTMC $V$ for the case $r \in U_{S}$. 
a state in $D$ ". We can formulate $\operatorname{IAVCD}(t, p)$ as

$$
\begin{aligned}
\operatorname{IAVCD}(t, p)= & \sum_{n=0}^{\infty}\left(\sum _ { k = 1 } ^ { n + 1 } P \left[\#\left(\hat{X}_{0: n} \in U\right)=k \wedge \hat{X}_{n} \in U \wedge \sum_{i=1}^{k-1} X_{i}^{U}+\sum_{i=1}^{n+1-k} X_{i}^{D}<t\right.\right. \\
& \left.\wedge \sum_{i=1}^{k} X_{i}^{U}+\sum_{i=1}^{n+1-k} X_{i}^{D} \geqslant t \wedge t-\sum_{i=1}^{n+1-k} X_{i}^{D}>p t\right] \\
& +\sum_{k=1}^{n} P\left[\#\left(\hat{X}_{0: n} \in U\right)=k \wedge \hat{X}_{n} \in D \wedge \sum_{i=1}^{k} X_{i}^{U}+\sum_{i=1}^{n-k} X_{i}^{D}<t\right. \\
& \left.\left.\wedge \sum_{i=1}^{k} X_{i}^{U}+\sum_{i=1}^{n+1-k} X_{i}^{D} \geqslant t \wedge \sum_{i=1}^{k} X_{i}^{U}>p t\right]\right) .
\end{aligned}
$$

The random variables $X_{i}^{U}$ and $X_{i}^{D}$ are independent among them and independent of $\hat{X}$. Then, we can formulate $\operatorname{IAVCD}(t, p)$ as

$$
\operatorname{IAVCD}(t, p)=\sum_{n=0}^{\infty}\left(\sum_{k=1}^{n+1} F_{U}(k, n+1-k, t, p t) W_{n, k}^{U}+\sum_{k=1}^{n} F_{D}(k, n+1-k, t, p t) W_{n, k}^{D}\right)
$$

where $W_{n, k}^{U}=P\left[\#\left(\hat{X}_{0: n} \in U\right)=k \wedge \hat{X}_{n} \in U\right], W_{n, k}^{D}=P\left[\#\left(\hat{X}_{0: n} \in U\right)=k \wedge \hat{X}_{n} \in D\right]$ and

$$
\begin{aligned}
& F_{U}(k, m, t, s)=P\left[\sum_{i=1}^{k-1} X_{i}^{U}+\sum_{i=1}^{m} X_{i}^{D}<t \wedge \sum_{i=1}^{k} X_{i}^{U}+\sum_{i=1}^{m} X_{i}^{D} \geqslant t \wedge t-\sum_{i=1}^{m} X_{i}^{D}>s\right], \\
& F_{D}(k, m, t, s)=P\left[\sum_{i=1}^{k} X_{i}^{U}+\sum_{i=1}^{m-1} X_{i}^{D}<t \wedge \sum_{i=1}^{k} X_{i}^{U}+\sum_{i=1}^{m} X_{i}^{D} \geqslant t \wedge \sum_{i=1}^{k} X_{i}^{U}>s\right] .
\end{aligned}
$$

Since $V$ can be interpreted as the derandomized CTMC of $\hat{V}$ with randomization rate $\Lambda_{U}$ in ${ }^{V} U$ and $\Lambda_{D}$ in ${ }^{V} D$, a formulation similar to (22) can be derived for the interval availability complementary distribution of $V$. Given the definition of $\tilde{V}$ (21), $\tilde{V}_{n} \in{ }^{V} U$ if and only if $\hat{X}_{n} \in U$ and $\tilde{V}_{n} \in{ }^{V} D$ if and only if $\hat{X}_{n} \in D$. This implies $\#\left(\tilde{V}_{0: n} \in{ }^{V} U\right)=k \wedge \tilde{V}_{n} \in{ }^{V} U$ if and only if $\#\left(\hat{X}_{0: n} \in U\right)=k \wedge \hat{X}_{n} \in U$ and $\#\left(\tilde{V}_{0: n} \in{ }^{V} U\right)=k \wedge \tilde{V}_{n} \in{ }^{V} D$ if and only if $\#\left(\hat{X}_{0: n} \in U\right)=k \wedge \hat{X}_{n} \in D$ and, then, using Proposition 2,

$$
\begin{aligned}
P\left[\#\left(\hat{V}_{0: n} \in{ }^{V} U\right)\right. & \left.=k \wedge \hat{V}_{n} \in{ }^{V} U\right]=P\left[\#\left(\tilde{V}_{0: n} \in{ }^{V} U\right)=k \wedge \tilde{V}_{n} \in{ }^{V} U\right] \\
& =P\left[\#\left(\hat{X}_{0: n} \in U\right)=k \wedge \hat{X}_{n} \in U\right], \\
P\left[\#\left(\hat{V}_{0: n} \in{ }^{V} U\right)\right. & \left.=k \wedge \hat{V}_{n} \in{ }^{V} D\right]=P\left[\#\left(\tilde{V}_{0: n} \in{ }^{V} U\right)=k \wedge \tilde{V}_{n} \in{ }^{V} D\right] \\
& =P\left[\#\left(\hat{X}_{0: n} \in U\right)=k \wedge \hat{X}_{n} \in D\right],
\end{aligned}
$$

which implies the theorem. 


\subsection{Truncation of the transformed model}

The transformed CTMC $V$ can have an infinite state space and, then, solving it exactly does not seem feasible. In this section, we will show how the state space of $V$ can be pruned to obtain a truncated transformed model with always finite state space having the same interval availability complementary distribution as $V$ with error upper bounded by $\varepsilon / 2$, where $\varepsilon$ is an arbitrarily small error control parameter. The method will then compute the interval availability complementary distribution of the truncated transformed model with error upper bounded by $\varepsilon / 2$ using Algorithm A of [6], yielding a total error in the computation of the interval availability complementary distribution of $X$ upper bounded by $\varepsilon$. The fact that $\hat{V}$ (and therefore $V$ ) has an infinite state space in the frequent case $U_{S}^{\prime} \neq \emptyset$ (which implies by condition C5 $\lambda_{r, U_{S}^{\prime}}>0$ and $P_{r, U_{S}^{\prime}}>0$ ) can be shown from $P_{i, i}>0, i \in U_{S}^{\prime}$ as follows. If $r \in U_{S}$, then state $s_{0,1}^{u}$ will belong to $\hat{V}$ and $P_{r, U_{S}^{\prime}}>0, P_{i, i}>0, i \in U_{S}^{\prime}$ implies the existence in $\hat{V}$ of the path $s_{0,1}^{u} \rightarrow s_{1,2}^{u} \rightarrow s_{2,3}^{u} \rightarrow s_{3,4}^{u} \rightarrow \cdots$, which has infinitely many different states. If $r \in D_{S}$, then state $s_{0,0}^{d}$ will belong to $\hat{V}$ and $P_{r, U_{S}^{\prime}}>0, P_{i, i}>0, i \in U_{S}^{\prime}$ implies the existence in $\hat{V}$ of the path $s_{0,0}^{d} \rightarrow s_{1,1}^{u} \rightarrow s_{2,2}^{u} \rightarrow s_{3,3}^{u} \rightarrow \cdots$, which also has infinitely many different states. It is also easy to show that, if $\alpha_{S^{\prime}}>0$ and $U_{S}^{\prime} \neq \emptyset, V$ has infinitely many different states $s_{n, k}^{\prime \prime}$, $s_{n, k}^{\prime d}$, for, if $\alpha_{U_{S}^{\prime}}>0, P_{i, i}>0, i \in U_{S}^{\prime}$ implies the existence in $V$ of the path $s_{0,1}^{\prime u} \rightarrow s_{1,2}^{\prime u} \rightarrow s_{2,3}^{\prime u} \rightarrow s_{3,4}^{\prime u} \rightarrow \cdots$ and, if $\alpha_{U_{S}^{\prime}}=0$, which implies $\alpha_{D_{S}^{\prime}}>0$ and, by condition C6, $\lambda_{i, U_{S}^{\prime}}>0, P_{i, U_{S}^{\prime}}>0$ for some $i \in D_{S}^{\prime}$ with $\alpha_{i}>0, P_{i, i}>0, i \in U_{S}^{\prime}$ implies the existence in $V$ of the path $s_{0,0}^{\prime d} \rightarrow s_{1,1}^{\prime \prime} \rightarrow s_{2,2}^{\prime u} \rightarrow s_{3,3}^{\prime u} \rightarrow \cdots$.

We will perform two truncations, in general. The first truncation yields a CTMC $V_{C}$ which is obtained from $V$ by deleting the states $s_{n, k}^{u}, s_{n, k}^{d}, s_{n, k}^{\prime u}$, and $s_{n, k}^{\prime d}$ with $k \leqslant n-C$, where $C \geqslant 1$ is the truncation parameter, and directing to an absorbing state $a$ the transition rates to states $s_{n, k}^{u}, s_{n, k}^{d}, s_{n, k}^{\prime u}$, and $s_{n, k}^{\prime d}$ with $k \leqslant n-C$. The state space of $V_{C}$ is $\Omega_{C} \cup\{a\}$, where $\Omega_{C}=\left\{s_{n, k}^{u}, s_{n, k}^{d}, s_{n, k}^{\prime u}, s_{n, k}^{\prime d} \in V \Omega\right.$ : $k \geqslant n-C+1\} \cup\{f\}$ and its initial probability distribution is the same as that of $V .{ }^{4}$ Formally, $V_{C}$ can be defined from $V$ as

$$
V_{C}(t)= \begin{cases}V(t) & \text { if } V(\tau) \in \Omega_{C} \text { for all } \tau \in[0, t] \\ a & \text { otherwise. }\end{cases}
$$

Note that, being $C \geqslant 1$, none of the states in the paths shown up in the previous paragraph will be deleted, showing that, for $U_{S}^{\prime} \neq \emptyset, V_{C}$ will have infinitely many different states $s_{n, k}^{u}, s_{n, k}^{d}$ and, if $\alpha_{S^{\prime}}>0$, infinitely many different states $s_{n, k}^{\prime u}, s_{n, k}^{\prime d}$. Let $\operatorname{IAVCD}_{C}^{a}(t, p)$ be the interval availability complementary distribution of $V_{C}$ with subset of up states, ${ }^{V} U_{C}$, including the states $s_{n, k}^{u}, s_{n, k}^{\prime \prime}$ and state $f$ if $f \in U$, and let $q=1-p$. Let ${ }^{V} D_{C}$ denote the subset of down states of $V_{C}\left({ }^{V} D_{C}\right.$ includes the states $s_{n, k}^{d}, s_{n, k}^{\prime d}$, the state $f$ if $f \in D$, and state $a$ ). Then, being $\Lambda=\max \left\{\Lambda_{U}, \Lambda_{D}\right\}$,

Theorem 2. $\operatorname{IAVCD}(t, p)=\operatorname{IAVCD}_{C}^{a}(t, p)+e_{C}^{1}(t, p)$ with $\left|e_{C}^{1}(t, p)\right| \leqslant \sum_{n=C+1}^{\infty} \mathrm{e}^{-\Lambda t q}(\Lambda t q)^{n} / n !$.

\footnotetext{
${ }^{4} V_{C}$ may not have transition rates to state $a$, and, then, state $a$ would be unreachable in $V_{C}$. A situation in which this would happen is when $D_{S}^{\prime}=\emptyset$. However, this is not a problem.
} 
Proof. Consider the randomized DTMCs $\hat{V}^{\prime}$ and $\hat{V}_{C}^{\prime}$ of, respectively, $V$ and $V_{C}$ with randomization rate $\Lambda$ in all states. The state transition diagram of $\hat{V}^{\prime}$ is similar to the state transition diagram of $\hat{V}$ (illustrated in Fig. 2), with the difference that all states $s_{n, k}^{u}$ and all states $s_{n, k}^{\prime u}$ will have non-null transition probabilities to themselves if $\Lambda_{D}>\Lambda_{U}$ and all states $s_{n, k}^{d}$ and all states $s_{n, k}^{\prime d}$ will have non-null transition probabilities to themselves if $\Lambda_{U}>\Lambda_{D}$. Fig. 4 sketches a feasible state transition diagram of $\hat{V}^{\prime}$ for the case $r \in U_{S}$ and $\Lambda_{U}>\Lambda_{D}$. The state transition diagram of $\hat{V}_{C}^{\prime}$ can be obtained from the state transition diagram of $\hat{V}^{\prime}$ by deleting the states $s_{n, k}^{u}, s_{n, k}^{d}, s_{n, k}^{\prime u}$ and $s_{n, k}^{\prime d}$ with $k \leqslant n-C$, and directing to an absorbing state $a$ the transitions to states $s_{n, k}^{u}, s_{n, k}^{d}, s_{n, k}^{\prime u}$ and $s_{n, k}^{\prime d}$ with $k \leqslant n-C$. $\hat{V}_{C}^{\prime}$ can be defined from $\hat{V}^{\prime}$ as

$$
\left(\hat{V}_{C}^{\prime}\right)_{n}= \begin{cases}\hat{V}_{n}^{\prime} & \text { if } \hat{V}_{0: n}^{\prime} \in \Omega_{C} \\ a & \text { otherwise }\end{cases}
$$

Using the formulation (1) for the interval availability complementary distribution on which Algorithm A of [6] is based, we have

$$
\begin{aligned}
\operatorname{IAVCD}(t, p) & =\sum_{n=0}^{\infty} \mathrm{e}^{-\Lambda t} \frac{(\Lambda t)^{n}}{n !} \sum_{k=0}^{n}\left(\begin{array}{l}
n \\
k
\end{array}\right) p^{k} q^{n-k} Y_{n, k} \\
& =\sum_{k=0}^{\infty} \sum_{n=k}^{\infty} \mathrm{e}^{-\Lambda t} \frac{(\Lambda t)^{n}}{n !}\left(\begin{array}{l}
n \\
k
\end{array}\right) p^{k} q^{n-k} Y_{n, k}, \\
\operatorname{IAVCD}_{C}^{a}(t, p) & =\sum_{k=0}^{\infty} \sum_{n=k}^{\infty} \mathrm{e}^{-\Lambda t} \frac{(\Lambda t)^{n}}{n !}\left(\begin{array}{l}
n \\
k
\end{array}\right) p^{k} q^{n-k} Y_{n, k}^{a},
\end{aligned}
$$

with $Y_{n, k}=P\left[\#\left(\hat{V}_{0: n}^{\prime} \in{ }^{V} U\right)>k\right]$ and $Y_{n, k}^{a}=P\left[\#\left(\left(\hat{V}_{C}^{\prime}\right)_{0: n} \in{ }^{V} U_{C}\right)>k\right]$. But, given the state transition diagram of $\hat{V}^{\prime}$ and the fact that its initial probability distribution is concentrated in states $s_{0,1}^{u}$, $s_{0,0}^{d}, \quad s_{0,1}^{\prime \prime}, s_{0,0}^{\prime d}$, and $f$, the fact that $\hat{V}^{\prime}$ has entered by step $n$ a state $s_{l, k}^{u}$, a state $s_{l, k}^{d}$, a state $s_{l, k}^{\prime u}$ or a state $s_{l, k}^{\prime d}$ implies $\#\left(\hat{V}_{0: n}^{\prime} \in{ }^{V} D\right) \geqslant l+1-k$ and, therefore, $\#\left(\hat{V}_{0: n}^{\prime} \in{ }^{V} U\right) \leqslant n-l+k$. Then, $\#\left(\hat{V}_{0: n}^{\prime} \in{ }^{V} U\right)>k$ implies that $\hat{V}^{\prime}$ has not entered by step $n$ a state $s_{l, k^{\prime}}^{u}$, a state $s_{l, k^{\prime}}^{d}$, a state $s_{l, k^{\prime}}^{\prime u}$, or a state $s_{l, k^{\prime}}^{\prime d}$ with $n-l+k^{\prime} \leqslant k$, and $\#\left(\hat{V}_{0: n}^{\prime} \in{ }^{V} U\right)=k>n-C$ implies that, by step $n$, $\hat{V}^{\prime}$ has not entered a state $s_{l, k^{\prime}}^{u}$, a state $s_{l, k^{\prime}}^{d}$, a state $s_{l, k^{\prime}}^{\prime u}$ or a state $s_{l, k^{\prime}}^{\prime d}$ with $n-l+k^{\prime} \leqslant n-C$, i.e. $k^{\prime} \leqslant l-C$, that, by step $n, \hat{V}^{\prime}$ has not left $\Omega_{C}$ and (23) that $\#\left(\left(\hat{V}_{C}^{\prime}\right)_{0: n} \in{ }^{V} U_{C}\right)=k$. It can be similarly shown that $\#\left(\left(\hat{V}_{C}^{\prime}\right)_{0: n} \in{ }^{V} U_{C}\right)=k>n-C$ implies $\#\left(\hat{V}_{0: n}^{\prime} \in{ }^{V} U\right)=k$. Then, for $k>n-C$, \#(( $\left.\left.\hat{V}_{C}^{\prime}\right)_{0: n} \in{ }^{V} U_{C}\right)=k$ if and only if $\#\left(\hat{V}_{0: n}^{\prime} \in{ }^{V} U\right)=k$, which implies $Y_{n, k}^{a}=Y_{n, k}$ for $k \geqslant n-C(n \leqslant C+k)$. Then, using (24), (25),

$$
e_{C}^{1}(t, p)=\sum_{k=0}^{\infty} \sum_{n=C+k+1}^{\infty} \mathrm{e}^{-\Lambda t} \frac{(\Lambda t)^{n}}{n !}\left(\begin{array}{l}
n \\
k
\end{array}\right) p^{k} q^{n-k}\left(Y_{n, k}-Y_{n, k}^{a}\right),
$$




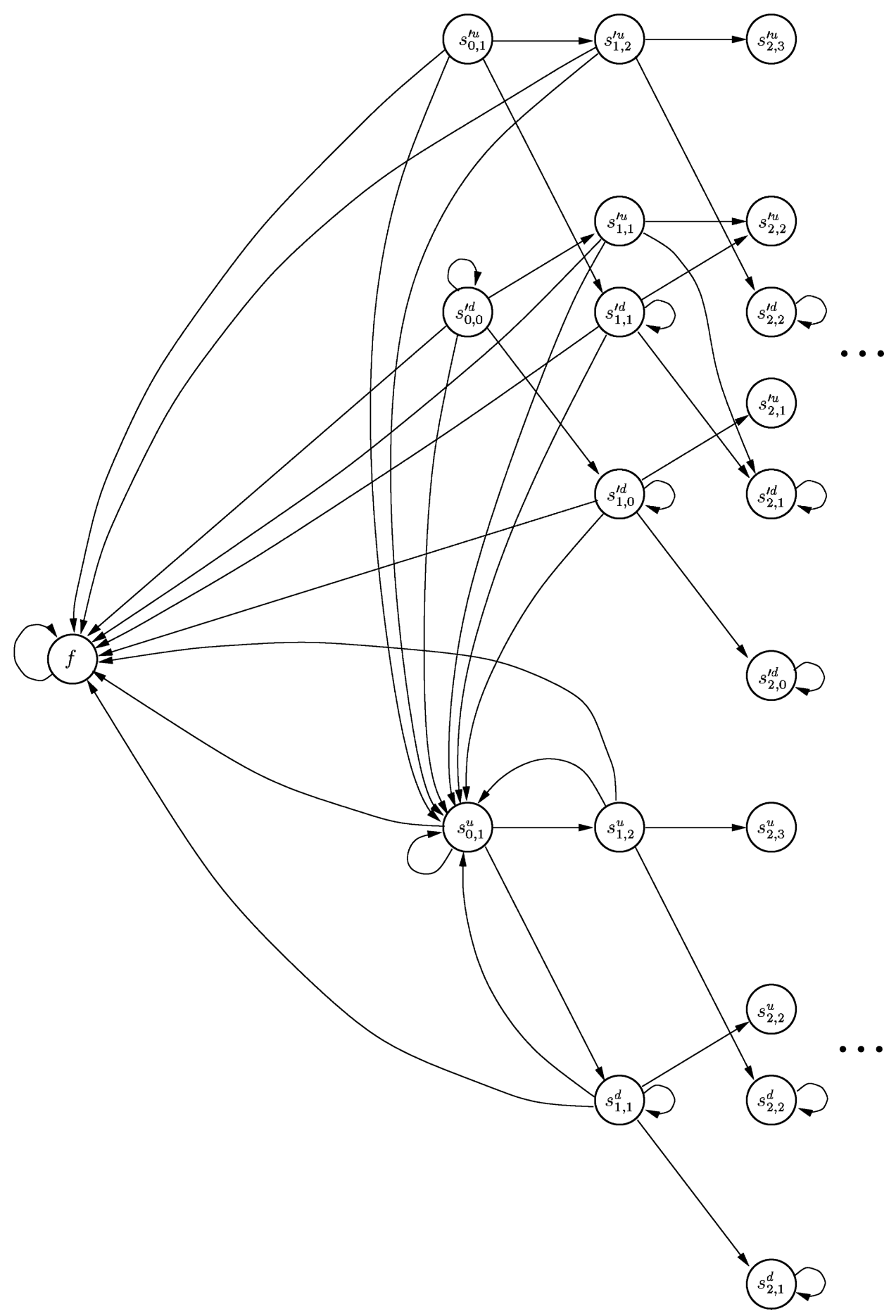

Fig. 4. Sketch of a feasible state transition diagram of the DTMC $\hat{V}^{\prime}$ for the case $r \in U_{S}$ and $\Lambda_{U}>\Lambda_{D}$. 
and, using $0 \leqslant Y_{n, k}, Y_{n, k}^{a} \leqslant 1$, which implies $\left|Y_{n, k}-Y_{n, k}^{a}\right| \leqslant 1$,

$$
\begin{aligned}
\left|e_{C}^{1}(t, p)\right| & \leqslant \sum_{k=0}^{\infty} \sum_{n=C+k+1}^{\infty} \mathrm{e}^{-\Lambda t} \frac{(\Lambda t)^{n}}{n !}\left(\begin{array}{l}
n \\
k
\end{array}\right) p^{k} q^{n-k}=\sum_{k=0}^{\infty} \mathrm{e}^{-\Lambda t p} \frac{(\Lambda t p)^{k}}{k !} \sum_{n=C+k+1}^{\infty} \mathrm{e}^{-\Lambda t q} \frac{(\Lambda t q)^{n-k}}{(n-k) !} \\
& =\sum_{k=0}^{\infty} \mathrm{e}^{-\Lambda t p} \frac{(\Lambda t p)^{k}}{k !} \sum_{n=C+1}^{\infty} \mathrm{e}^{-\Lambda t q} \frac{(\Lambda t q)^{n}}{n !}=\sum_{n=C+1}^{\infty} \mathrm{e}^{-\Lambda t q} \frac{(\Lambda t q)^{n}}{n !} \cdot
\end{aligned}
$$

In the particular case $U_{S}^{\prime}=\emptyset$, besides $f, \Omega_{C}$ only includes states $s_{0,0}^{\prime d}, s_{1,0}^{\prime d}, \ldots, s_{C-1,0}^{\prime d}$ if $\alpha_{S^{\prime}}>0$, state $s_{0,1}^{u}$ if $r \in U_{S}$, states $s_{1,1}^{d}, s_{2,1}^{d}, \ldots, s_{C, 1}^{d}$ if $r \in U_{S}$ and $\lambda_{r, D_{S}^{\prime}}>0$, state $s_{0,0}^{d}$ if $r \in D_{S}$, and states $s_{1,0}^{d}, s_{2,0}^{d}, \ldots, s_{C-1,0}^{d}$ if $r \in D_{S}$ and $\lambda_{r, D_{S}^{\prime}}>0$. Therefore, in the case $U_{S}^{\prime}=\emptyset, V_{C}$ has always a finite state space and, in that case, we do not perform a second truncation.

Assuming $U_{S}^{\prime} \neq \emptyset$, the second truncation is performed over the CTMC $V_{C}$ and yields a CTMC $V_{C, K, L}, K \geqslant 2, L \geqslant 2$, if $\Omega_{C}$ includes states $s_{n, k}^{\prime u}, s_{n, k}^{\prime d}$ (which happens if $\alpha_{S^{\prime}}>0$ ) and a CTMC $V_{C, K}, K \geqslant 2$, if $\alpha_{S^{\prime}}=0$ and $\Omega_{C}$ does not include any state $s_{n, k}^{\prime u}, s_{n, k}^{\prime d}$. The CTMC $V_{C, K, L}$ is obtained from $V_{C}$ by deleting the states $s_{n, k}^{u}, s_{n, k}^{d}$ with $k>K$ and the states $s_{n, k}^{\prime u}, s_{n, k}^{\prime d}$ with $k>L$ and directing to an absorbing state $b$ the transition rates from states $s_{n, K}^{u}, s_{n, K}^{d}, s_{n, L}^{\prime u}$ and $s_{n, L}^{\prime d}$. The CTMC $V_{C, K}$ is obtained from $V_{C}$ by deleting the states $s_{n, k}^{u}, s_{n, k}^{d}$ with $k>K$ and directing to an absorbing state $b$ the transition rates from states $s_{n, K}^{u}, s_{n, K}^{d}$. The state space of $V_{C, K, L}$ is $\Omega_{C, K, L} \cup\{a, b\}$, where $\Omega_{C, K, L}=\left\{s_{n, k}^{u}, s_{n, k}^{d} \in{ }^{V} \Omega: k \geqslant n-C+1 \wedge k \leqslant K\right\} \cup\left\{s_{n, k}^{\prime \prime}, s_{n, k}^{\prime d} \in{ }^{V} \Omega: k \geqslant n-C+1 \wedge k \leqslant L\right\} \cup\{f\}$ and its initial probability distribution is the same as that of $V$. Formally, $V_{C, K, L}$ can be defined from $V_{C}$ as

$$
V_{C, K, L}(t)= \begin{cases}V_{C}(t) & \text { if, by time } t, V_{C} \text { has not exited a state } s_{n, K}^{u}, s_{n, K}^{d}, s_{n, L}^{\prime u} \text { or } s_{n, L}^{\prime d} \\ b & \text { otherwise. }\end{cases}
$$

The state space of $V_{C, K}$ is $\Omega_{C, K} \cup\{a, b\}$, where $\Omega_{C, K}=\left\{s_{n, k}^{u}, s_{n, k}^{d} \in{ }^{V} \Omega: k \geqslant n-C+1 \wedge k \leqslant K\right\} \cup\{f\}$ and its initial probability distribution is the same as that of $V$. Formally, $V_{C, K}$ can be defined from $V_{C}$ as

$$
V_{C, K}(t)= \begin{cases}V_{C}(t) & \text { if, by time } t, V_{C} \text { has not exited a state } s_{n, K}^{u} \text { or } s_{n, K}^{d}, \\ b & \text { otherwise. }\end{cases}
$$

For the case $\alpha_{S^{\prime}}>0$, let $\operatorname{IAVCD}_{C, K, L}^{a}(t, p)$ be the interval availability complementary distribution of $V_{C, K, L}$ with subset of up states, ${ }^{V} U_{C, K, L}$, including the states $s_{n, k}^{u}, s_{n, k}^{\prime u}$ and state $f$ if $f \in U$, and let ${ }^{V} D_{C, K, L}$ denote the subset of down states of $V_{C, K, L}\left({ }^{V} D_{C, K, L}\right.$ includes the states $s_{n, k}^{d}, s_{n, k}^{\prime d}$, state $f$ if $f \in D$, and states $a$ and $b$ ). For the case $\alpha_{S^{\prime}}=0$, let $\operatorname{IAVCD}_{C, K}^{a}(t, p)$ be the interval availability complementary distribution of $V_{C, K}$ with subset of up states, ${ }^{V} U_{C, K}$, including the states $s_{n, k}^{u}$ and state $f$ if $f \in U$, and let ${ }^{V} D_{C, K}$ denote the subset of down states of $V_{C, K}\left({ }^{V} D_{C, K}\right.$ includes the states $s_{n, k}^{d}$, state $f$ if $f \in D$, and states $a$ and $b$ ). We have 
Proposition 3. Assume $U_{S}^{\prime} \neq \emptyset$. For the case $\alpha_{S^{\prime}}>0, \operatorname{IAVCD}_{C}^{a}(t, p)=\operatorname{IAVCD}_{C, K, L}^{a}(t, p)+$ $e_{C, K, L}^{2}(t, p) \quad$ with $\left|e_{C, K, L}^{2}(t, p)\right| \leqslant P\left[V_{C, K, L}(t)=b\right]$. For the case $\alpha_{S^{\prime}}=0$, $\operatorname{IAVCD}_{C}^{a}(t, p)=$ $\operatorname{IAVCD}_{C, K}^{a}(t, p)+e_{C, K}^{2}(t, p)$ with $\left|e_{C, K}^{2}(t, p)\right| \leqslant P\left[V_{C, K}(t)=b\right]$.

Proof. Consider the case $\alpha_{S^{\prime}}>0$, let $A(t)$ be the proposition "by time $t, V_{C}$ has not exited a state $s_{n, K}^{u}, s_{n, K}^{d}, s_{n, L}^{\prime u}$ or $s_{n, L}^{\prime d}$ " and let $\overline{A(t)}$ denote the negated proposition of $A(t)$. Conditioning on $V_{C}(t)$ and whether or not $A(t)$ holds, and using the fact that $A(t)$ implies $V_{C}(t) \in \Omega_{C, K, L} \cup\{a\}$ :

$$
\begin{aligned}
\operatorname{IAVCD}_{C}^{a}(t, p)= & \sum_{i \in \Omega_{C, K, L} \cup\{a\}} P\left[V_{C}(t)=i \wedge A(t)\right] P\left[\int_{0}^{t} I_{V_{C}(\tau) \in U^{V} U_{C}} \mathrm{~d} \tau>p t \mid V_{C}(t)=i \wedge A(t)\right] \\
& +\sum_{i \in \Omega_{C} \cup\{a\}} P\left[V_{C}(t)=i \wedge \overline{A(t)}\right] P\left[\int_{0}^{t} I_{V_{C}(\tau) \in{ }^{V} U_{C}} \mathrm{~d} \tau \mid V_{C}(t)=i \wedge \overline{A(t)}\right] .
\end{aligned}
$$

Similarly, conditioning on $V_{C, K, L}(t)$ :

$$
\begin{aligned}
\operatorname{IAVCD}_{C, K, L}^{a}(t, p)= & \sum_{i \in \Omega_{C, K, L} \cup\{a\}} P\left[V_{C, K, L}(t)=i\right] P\left[\int_{0}^{t} I_{V_{C, K, L}(\tau) \in V} U_{C, K, L}\right. \\
& \left.\mathrm{d} \tau>p t \mid V_{C, K, L}(t)=i\right] \\
& +P\left[V_{C, K, L}(t)=b\right] P\left[\int_{0}^{t} I_{V_{C, K, L}(\tau) \in V^{V} U_{C, K, L}} \mathrm{~d} \tau>p t \mid V_{C, K, L}(t)=b\right] .
\end{aligned}
$$

But, according to (26), $V_{C, K, L}(t) \in \Omega_{C, K, L} \cup\{a\}$ if and only if $A(t)$ holds and, in that case, $V_{C, K, L}(\tau)=$ $V_{C}(\tau), \tau \in[0, t]$. This together with ${ }^{V} U_{C, K, L}={ }^{V} U_{C} \cap \Omega_{C, K, L}$ implies $P\left[V_{C, K, L}(t)=i\right]=P\left[V_{C}(t)=i \wedge\right.$ $A(t)], \quad i \in \Omega_{C, K, L} \cup\{a\}$ and $P\left[\int_{0}^{t} I_{V_{C, K, L}(\tau) \in V^{V} U_{C, K, L}} \mathrm{~d} \tau>p t \mid V_{C, K, L}(t)=i\right]=P\left[\int_{0}^{t} I_{V_{C}(\tau) \in U^{V} U_{C}} \mathrm{~d} \tau>p t \mid\right.$ $\left.V_{C}(t)=i \wedge A(t)\right], i \in \Omega_{C, K, L} \cup\{a\}$, and, then,

$$
\begin{aligned}
e_{C, K, L}^{2}(t, p)= & \operatorname{IAVCD}_{C}^{a}(t, p)-\operatorname{IAVCD}_{C, K, L}^{a}(t, p) \\
= & \sum_{i \in \Omega_{C} \cup\{a\}} P\left[V_{C}(t)=i \wedge \overline{A(t)}\right] P\left[\int_{0}^{t} I_{V_{C}(\tau) \in V^{V} U_{C}} \mathrm{~d} \tau>p t \mid V_{C}(t)=i \wedge \overline{A(t)}\right] \\
& -P\left[V_{C, K, L}(t)=b\right] P\left[\int_{0}^{t} I_{V_{C, K, L}(\tau) \in V^{V} U_{C, K, L}} \mathrm{~d} \tau>p t \mid V_{C, K, L}(t)=b\right] .
\end{aligned}
$$

But, since $V_{C, K, L}(t)=b$ if and only if $\overline{A(t)}$ holds, $P\left[V_{C, K, L}(t)=b\right]=\sum_{i \in \Omega_{C} \cup\{a\}} P\left[V_{C}(t)=i \wedge \overline{A(t)}\right]$ and, using $0 \leqslant P\left[\int_{0}^{t} I_{V_{C}(\tau) \in{ }^{V} U_{C}} \mathrm{~d} \tau>p t \mid V_{C}(t)=i \wedge \overline{A(t)}\right], P\left[\int_{0}^{t} I_{V_{C, K, L}(\tau) \in^{V} U_{C, K, L}} \mathrm{~d} \tau>p t \mid V_{C, K, L}(t)=b\right]$ $\leqslant 1$, it is easy to show $\left|e_{C, K, L}^{2}(t, p)\right| \leqslant P\left[V_{C, K, L}(t)=b\right]$. The fact that $\left|e_{C, K}^{2}(t, p)\right| \leqslant P\left[V_{C, K}(t)\right.$ $=b]$ for the case $\alpha_{S^{\prime}}=0$ can be proved similarly.

According to Proposition 3, the absolute error in the interval availability complementary distribution introduced by the second truncation can be upper bounded by upper bounding $P\left[V_{C, K, L}(t)=b\right]$, if $\alpha_{S^{\prime}}>0$, and $P\left[V_{C, K}(t)=b\right]$, if $\alpha_{S^{\prime}}=0$. We will derive next upper bounds for those probabilities. For the case $\alpha_{S^{\prime}}>0$, let $\hat{V}_{C, K, L}$ be the randomized DTMC of $V_{C, K, L}$ with randomization rate $\Lambda_{U}$ in ${ }^{V} U_{C, K, L}$ 
and $\Lambda_{D}$ in ${ }^{V} D_{C, K, L}$. For the case $\alpha_{S^{\prime}}=0$, let $\hat{V}_{C, K}$ be the randomized DTMC of $V_{C, K}$ with randomization rate $\Lambda_{U}$ in ${ }^{V} U_{C, K}$ and $\Lambda_{D}$ in ${ }^{V} D_{C, K}$. Consider first the case $\alpha_{S^{\prime}}>0$ and let $\phi_{C, K, L}(m)$ be the probability that $\hat{V}_{C, K, L}$ enters $b$ after $m$ visits to states $s_{n, k}^{u}, s_{n, k}^{\prime u}$. Note that $\phi_{C, K, L}(m)=0$ for $m<\min \{K, L\}$. Using the interpretation of $V_{C, K, L}$ as randomized CTMC of $\hat{V}_{C, K, L}$ with randomization rate $\Lambda_{U}$ in ${ }^{V} U_{C, K, L}$ and $\Lambda_{D}$ in ${ }^{V} D_{C, K, L}$ and taking into account that the probability that the sum of $k$ independent exponential random variables with parameter $\Lambda_{U}$ is $\leqslant t$ is $\sum_{n=k}^{\infty} \mathrm{e}^{-\Lambda_{U} t}\left(\Lambda_{U} t\right)^{n} / n$ !, it is clear that

$$
P\left[V_{C, K, L}(t)=b\right] \leqslant \sum_{k=\min \{K, L\}}^{\infty} \phi_{C, K, L}(k) \sum_{n=k}^{\infty} \mathrm{e}^{-\Lambda_{U} t} \frac{\left(\Lambda_{U} t\right)^{n}}{n !} .
$$

Let $b_{C, L}(k)$ be the probability that $\hat{V}_{C, K, L}$ has entered $b$ through a state $s_{n, L}^{\prime u}$ or a state $s_{n, L}^{\prime d}$ after no more than $k$ visits to states $s_{n, l}^{u}, s_{n, l}^{\prime \prime}$. Let $c_{C, K, L}(k)$ be the probability that $\hat{V}_{C, K, L}$ has entered $b$ through a state $s_{n, K}^{u}$ or a state $s_{n, K}^{d}$ after no more than $k$ visits to states $s_{n, l}^{u}, s_{n, l}^{\prime u}$. Note that $b_{C, L}(k)=0$ for $k<L$ and $c_{C, K, L}(k)=0$ for $k<K$. Also, $\phi_{C, K, L}(k)=b_{C, L}(k)-b_{C, L}(k-1)+c_{C, K, L}(k)-c_{C, K, L}(k-1)$, $k>0$. Then, using (27),

$$
\begin{aligned}
P\left[V_{C, K, L}(t)=\right. & b] \\
\leqslant & \sum_{k=}^{\infty} \min \{K, L\} \\
= & \sum_{k=L}^{\infty} b_{C, L}(k) \sum_{n=k}^{\infty} \mathrm{e}^{-\Lambda_{U} t} \frac{\left(\Lambda_{U} t\right)^{n}}{n !}-\sum_{k=L}^{\infty} b_{C, L}(k) \sum_{n=k+1}^{\infty} \mathrm{e}^{-\Lambda_{U} t} \frac{\left(\Lambda_{U} t\right)^{n}}{n !} \\
& +\sum_{k=K}^{\infty} c_{C, K, L}(k) \sum_{n=k}^{\infty} \mathrm{e}^{-\Lambda_{U} t} \frac{\left(\Lambda_{U} t\right)^{n}}{n !}-\sum_{k=K}^{\infty} c_{C, K, L}(k) \sum_{n=k+1}^{\infty} \mathrm{e}^{-\Lambda_{U} t} \frac{\left(\Lambda_{U} t\right)^{n}}{n !} \frac{\left(\Lambda_{U} t\right)^{n}}{n !} \\
= & \sum_{k=L}^{\infty} b_{C, L}(k) \mathrm{e}^{-\Lambda_{U} t} \frac{\left(\Lambda_{U} t\right)^{k}}{k !}+\sum_{k=K}^{\infty} c_{C, K, L}(k) \mathrm{e}^{-\Lambda_{U} t} \frac{\left(\Lambda_{U} t\right)^{k}}{k !}
\end{aligned}
$$

For the case $\alpha_{S^{\prime}}=0, \hat{V}_{C, K}$ does not include states $s_{n, k}^{\prime u}, s_{n, k}^{\prime d}$. In that case, it can be proved similarly that

$$
P\left[V_{C, K}(t)=b\right] \leqslant \sum_{k=K}^{\infty} c_{C, K}(k) \mathrm{e}^{-\Lambda_{U} t} \frac{\left(\Lambda_{U} t\right)^{k}}{k !}
$$

where $c_{C, K}(k)$ is the probability that $\hat{V}_{C, K}$ has entered $b$ after no more than $k$ visits to states $s_{n, l}^{u}$.

At this point, it is useful to clarify the domain of $(n, k)$ pairs for which some state $s_{n, k}^{u}$ or $s_{n, k}^{d}$ may belong to $\Omega_{C, K, L}\left(\Omega_{C, K}\right)$ and the domain of $(n, k)$ pairs for which some state $s_{n, k}^{\prime u}$ or $s_{n, k}^{\prime d}$ may belong to $\Omega_{C, K, L}$. The first domain is obtained by imposing $n-C+1 \leqslant k \leqslant n+1, n \geqslant 0$ and $0 \leqslant k \leqslant K$ and is shown in Fig. 5. The second domain is obtained by imposing $n-C+1 \leqslant k \leqslant n+1, n \geqslant 0$ and $0 \leqslant k \leqslant L$ and is identical to the first one with $K$ replaced by $L$. 


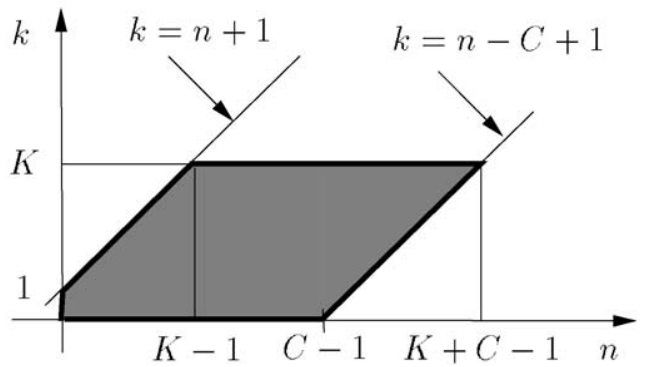

Fig. 5. Domain of $(n, k)$ pairs for which some state $s_{n, k}^{u}$ or $s_{n, k}^{d}$ may belong to $\Omega_{C, K, L}\left(\Omega_{C, K}\right)$ (the domain includes the points in the frontier).

Given the structure of the state transition diagram of $\hat{V}_{C, K, L}$ and its initial probability distribution, $\hat{V}_{C, K, L}$ can only enter $b$ through a state $s_{n, L}^{\prime u}$ or a state $s_{n, L}^{\prime d}$ following $L$ visits to states $s_{n, k}^{u}, s_{n, k}^{\prime \prime}$, and this will happen with the probability that $\hat{V}_{C, K, L}$ ever visits a state $s_{n, L}^{\prime \prime}$ or a state $s_{n, L}^{\prime d}$, equal to the probability that $\hat{V}_{C}$ ever visits a state $s_{n, L}^{\prime u}$ or a state $s_{n, L}^{\prime d}$, equal to the probability, $P$, that $\hat{V}$ ever visits a state $s_{n, L}^{\prime u}$ or a state $s_{n, L}^{\prime d}, n-C+1 \leqslant L \leqslant n+1$, i.e. $L-1 \leqslant n \leqslant L+C-1$. Since $\hat{V}$ can only be at a state $s_{n, k}^{\prime u}$ or a state $s_{n, k}^{\prime d}$ at step $n$, using the definition of $\hat{V}$ (21) and the definition of $Z^{\prime}(3), P \leqslant \sum_{n=L-1}^{L+C-1} P\left[\hat{X}_{0: n} \in S^{\prime} \wedge \#\left(\hat{X}_{0: n} \in U_{S}^{\prime}\right)=L\right]=\sum_{n=L-1}^{L+C-1} P\left[Z_{n}^{\prime} \in S^{\prime} \wedge\right.$ $\left.\#\left(Z_{0: n}^{\prime} \in U_{S}^{\prime}\right)=L\right]=\sum_{n=L-1}^{L+C-1} \sum_{i \in S^{\prime}} \pi_{i}^{\prime}(n, L)$. Then,

$$
b_{C, L}(k) \leqslant I_{k \geqslant L} a_{C}^{\prime}(L)
$$

with $a_{C}^{\prime}(L)=\sum_{n=L-1}^{L+C-1} \sum_{i \in S^{\prime}} \pi_{i}^{\prime}(n, L)$. The following proposition gives simple upper bounds for $c_{C, K, L}(k)$ and $c_{C, K}(k)$.

Proposition 4. Assume $U_{S}^{\prime} \neq \emptyset$. Let $a_{C}(K)=\sum_{n=K-1}^{K+C-1} \sum_{i \in S} \pi_{i}(n, K)$. Then, for the case $\alpha_{S^{\prime}}>0$, $c_{C, K, L}(k) \leqslant I_{k} \geqslant K-1 \alpha_{S}(k-K+2) a_{C}(K)$ and, for the case $\alpha_{S^{\prime}}=0, c_{C, K}(k) \leqslant I_{k} \geqslant K-1 \alpha_{S}(k-K+2) a_{C}(K)$.

Proof. See the Appendix A.

Proposition 3, (28)-(30) and the upper bounds for $c_{C, K, L}(k)$ and $c_{C, K}(k)$ given by Proposition 4 yield simple upper bounds for the error introduced by the second truncation:

Theorem 3. Assume $U_{S}^{\prime} \neq \emptyset$. Let $a_{C}(K)=\sum_{n=K-1}^{K+C-1} \sum_{i \in S} \pi_{i}(n, K)$ and $a_{C}^{\prime}(L)=\sum_{n=L-1}^{L+C-1} \sum_{i \in S^{\prime}} \pi_{i}^{\prime}(n, L)$. Then, for the case $\alpha_{S^{\prime}}>0,\left|e_{C, K, L}^{2}(t, p)\right| \leqslant a_{C}^{\prime}(L) \sum_{k=L}^{\infty} \mathrm{e}^{-\Lambda_{U} t}\left(\Lambda_{U} t\right)^{k} / k !+\alpha_{S} a_{C}(K) \sum_{k=K}^{\infty}(k-K+$ $2) \mathrm{e}^{-\Lambda_{U} t}\left(\Lambda_{U} t\right)^{k} / k !$ and, for the case $\alpha_{S}^{\prime}=0,\left|e_{C, K}^{2}(t, p)\right| \leqslant \alpha_{S} a_{C}(K) \sum_{k=K}^{\infty}(k-K+2) \mathrm{e}^{-\Lambda_{U} t}\left(\Lambda_{U} t\right)^{k} / k !$.

Using Theorems 2 and 3, the truncation parameters $C, K$ and $L$ can be selected as follows, guaranteeing that the truncated transformed model will have the same interval availability complementary 
distribution as $X$ with error upper bounded by $\varepsilon / 2$. For $C$, we can take

$$
C=\min \left\{c \geqslant 1: \sum_{k=c+1}^{\infty} \mathrm{e}^{-\Lambda t q} \frac{(\Lambda t q)^{k}}{k !} \leqslant \frac{\varepsilon}{2}\right\} \quad \text { if } U_{S}^{\prime}=\emptyset
$$

and

$$
C=\min \left\{c \geqslant 1: \sum_{k=c+1}^{\infty} \mathrm{e}^{-\Lambda t q} \frac{(\Lambda t q)^{k}}{k !} \leqslant \frac{\varepsilon}{4}\right\} \quad \text { if } U_{S}^{\prime} \neq \emptyset .
$$

Then, assuming $U_{S}^{\prime} \neq \emptyset$, for the case $\alpha_{S^{\prime}}>0$, we can take

$$
\begin{aligned}
& K=\min \left\{n \geqslant 2: \alpha_{S} a_{C}(n) \sum_{k=n}^{\infty}(k-n+2) \mathrm{e}^{-\Lambda_{U} t} \frac{\left(\Lambda_{U} t\right)^{k}}{k !} \leqslant \frac{\varepsilon}{8}\right\}, \\
& L=\min \left\{n \geqslant 2: a_{C}^{\prime}(n) \sum_{k=n}^{\infty} \mathrm{e}^{-\Lambda_{U} t} \frac{\left(\Lambda_{U} t\right)^{k}}{k !} \leqslant \frac{\varepsilon}{8}\right\},
\end{aligned}
$$

and, for the case $\alpha_{S^{\prime}}=0$, we can take

$$
K=\min \left\{n \geqslant 2: \alpha_{S} a_{C}(n) \sum_{k=n}^{\infty}(k-n+2) \mathrm{e}^{-\Lambda_{U} t} \frac{\left(\Lambda_{U} t\right)^{k}}{k !} \leqslant \frac{\varepsilon}{4}\right\} .
$$

\subsection{Algorithmic description and implementation details}

Figs. 6-8 give an algorithmic description of the method for the computation of the interval availability complementary distribution developed in this paper. The algorithm has as inputs the CTMC $X$, an absorb variable which has the value true when the state space of $X$ has to be seen as $\Omega=S \cup\{f\}$ and the value false when has to be seen as $\Omega=S$, the subset of up states $U$, the initial probability distribution row vector $\alpha$, the regenerative state $r \in S$, the allowed computation error $\varepsilon$, the number $n$ of pairs $(t, p)$ at which $\operatorname{IAVCD}(t, p)$ has to be computed, and the $(t, p)$ pairs, $\left(t_{1}, p_{1}\right),\left(t_{2}, p_{2}\right), \ldots,\left(t_{n}, p_{n}\right)$. The outputs of the algorithm are the computed values of $\operatorname{IAVCD}(t, p)$, $\operatorname{IAVCD}\left(t_{1}, p_{1}\right), \operatorname{IAVCD}\left(t_{2}, p_{2}\right), \ldots, \operatorname{IAVCD}\left(t_{n}, p_{n}\right)$, at the given $(t, p)$ pairs. It is assumed that, if $\Omega=S \cup\{f\}$, state $f$ has been identified and that $X$ satisfies the required conditions with the given selection for the regenerative state $r$. The truncated transformed model, called $V$ in the algorithmic description, is generated by adding states and transition rates to the initially empty CTMC $V$ using the functions add_state $(s, x)$ and add_transition $\left(s, s^{\prime}, \lambda\right)$. The first function adds a state $s$ with initial probability $x$ to $V$; the second function adds to $V$ a transition rate $\lambda$ from state $s$ to state $s^{\prime}$. The required truncation parameter $C$ increases with $t q=t(1-p)$ and, therefore, that parameter is selected using the largest value of $t q$ for which $\operatorname{IAVCD}(t, p)$ has to be computed. Once the required $C$ has been determined and for the case $U_{S}^{\prime} \neq \emptyset$, the CTMC $V$ is constructed by adding first states $s_{n, k}^{u}, s_{n, k}^{d}$, $\max \{0, k-1\} \leqslant n \leqslant k+C-1$ (see Fig. 5), for increasing $k$, controlling the error associated with the truncation parameter $K$ for $t=t_{\max }=\max _{1 \leqslant i \leqslant n} t_{i}$, since the model truncation error upper bounds associated with that parameter given by Theorem 3 increase with $t$. After that, if $\alpha_{S^{\prime}}>0$, states 


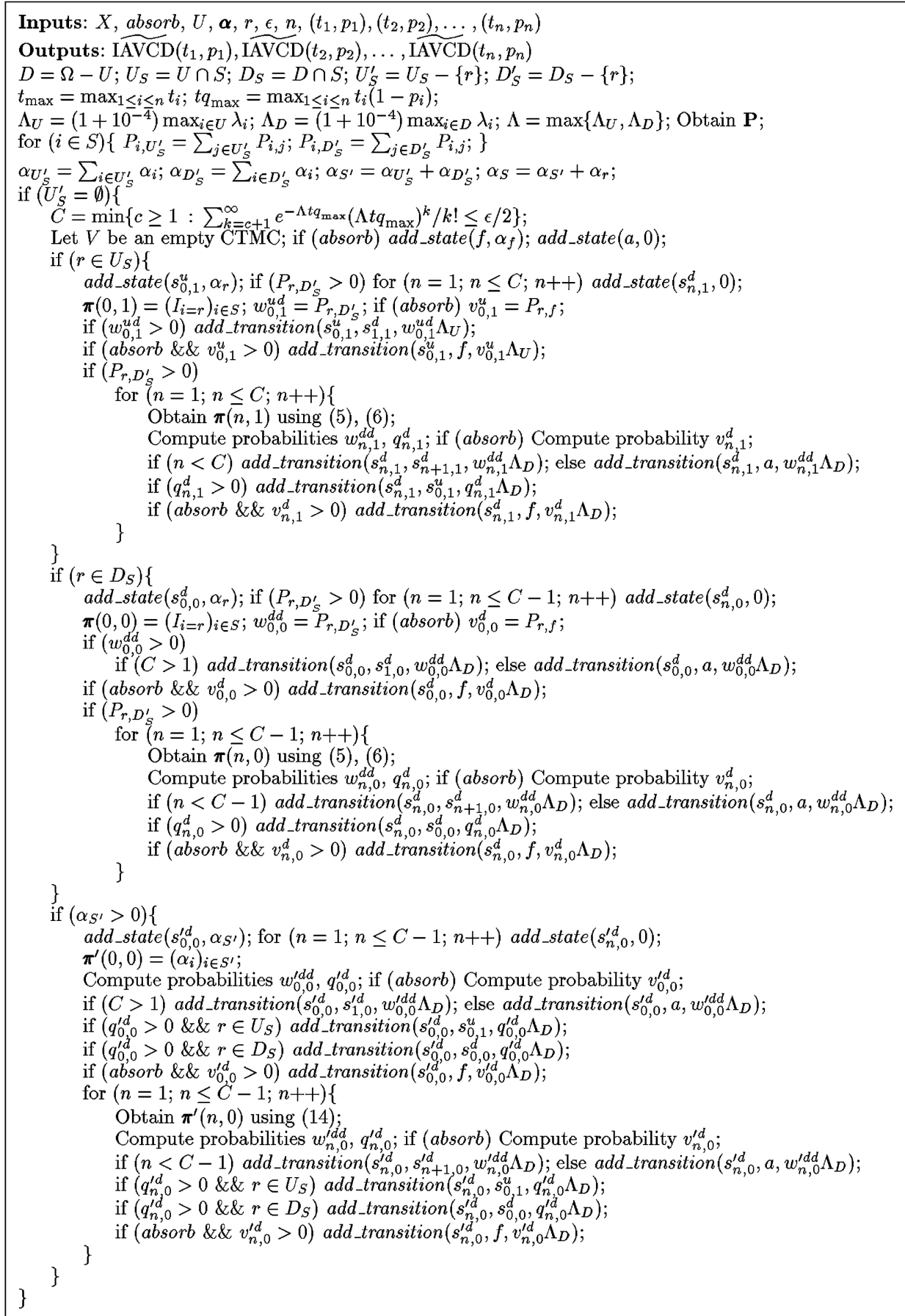

Fig. 6. Algorithmic description of the method. 


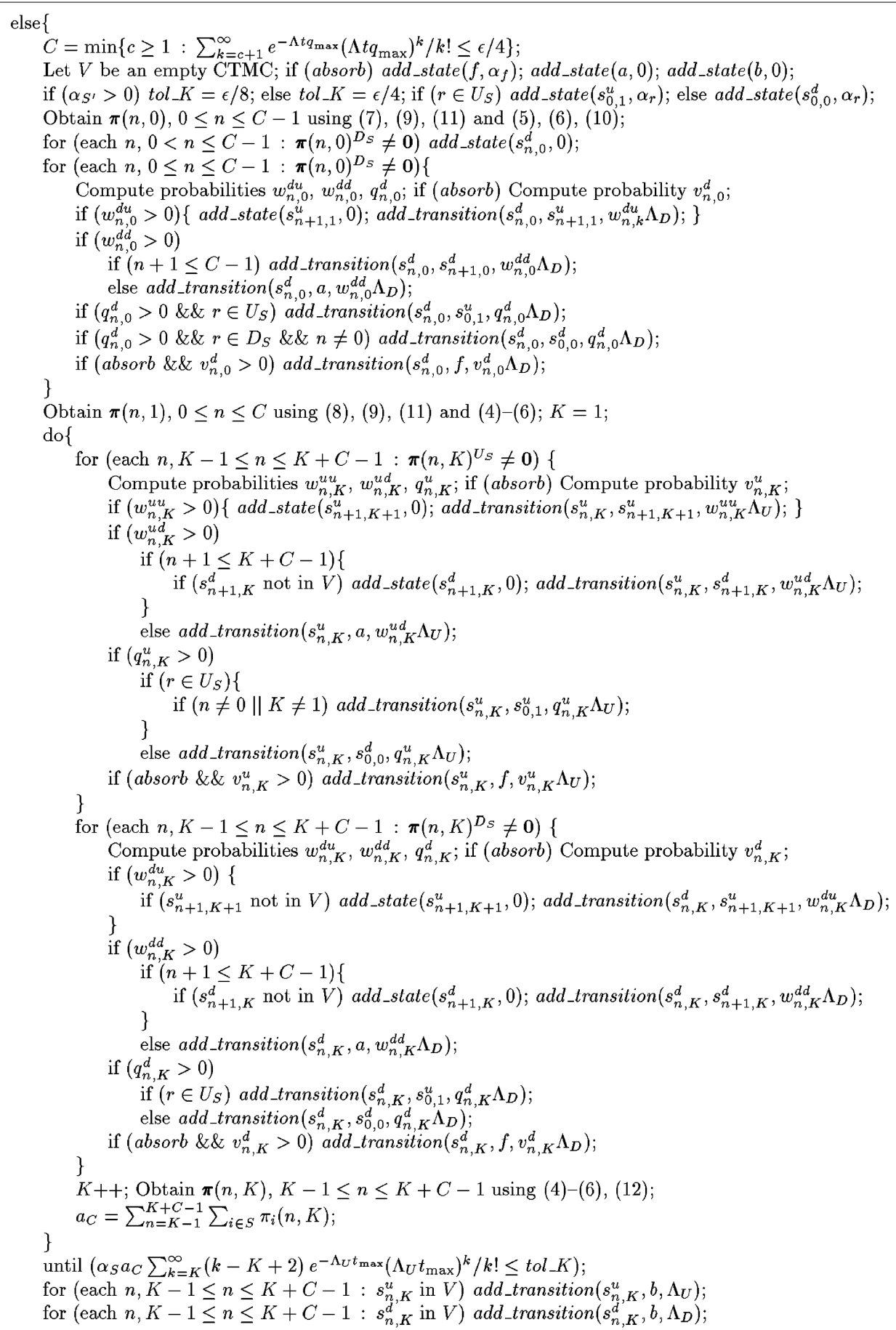

Fig. 7. Algorithmic description of the method (continuation). 
if $\left(\alpha_{S^{\prime}}>0\right)\{$

Obtain $\boldsymbol{\pi}^{\prime}(n, 0), 0 \leq n \leq C-1$ using (15), (18) and (14), (17);

for (each $n, 0 \leq n \leq C-1: \boldsymbol{\pi}^{\prime}(n, 0)^{D_{S}^{\prime}} \neq \mathbf{0}$ )

if $(n==0)$ add_state $\left(s_{0,0}^{\prime d}, \alpha_{D_{s}^{\prime}}\right)$; else add_state $\left(s_{n, 0}^{\prime d}, 0\right)$;

for (each $\left.n, 0 \leq n \leq C-1: \pi^{\prime}(n, 0)^{D_{s}^{\prime}} \neq \mathbf{0}\right)\{$

Compute probabilities $w_{n, 0}^{\prime d u}, w_{n, 0}^{\prime d d}, q_{n, 0}^{\prime d}$; if (absorb) Compute probability $v_{n, 0}^{\prime d}$;

if $\left(w_{n, 0}^{\prime d u}>0\right)\left\{\right.$ add_state $\left(s_{n+1,1}^{\prime u}, 0\right) ;$ add_transition $\left.\left(s_{n, 0}^{\prime d}, s_{n+1,1}^{\prime u}, w_{n, 0}^{\prime d u} \Lambda_{D}\right) ;\right\}$

if $\left(w_{n, 0}^{\prime d d}>0\right)$

if $(n+1 \leq C-1)$ add_transition $\left(s_{n, 0}^{\prime d}, s_{n+1,0}^{\prime d}, w_{n, 0}^{\prime d d} \Lambda_{D}\right)$;

else add_transition $\left(s_{n, 0}^{\prime d}, a, w_{n, 0}^{\prime d d} \Lambda_{D}\right)$;

if $\left(q_{n, 0}^{\prime d}>0 \& \& r \in U_{S}\right)$ add_transition $\left(s_{n, 0}^{\prime d}, s_{0,1}^{u}, q_{n, 0}^{\prime d} \Lambda_{D}\right)$;

if $\left(q_{n, 0}^{\prime d}>0 \& \& r \in D_{S}\right)$ add_transition $\left(s_{n, 0}^{\prime d}, s_{0,0}^{d}, q_{n, 0}^{\prime d} \Lambda_{D}\right)$;

\}

if $\left(a b s o r b \& \& v_{n, 0}^{\prime d}>0\right)$ add_transition $\left(s_{n, 0}^{\prime d}, f, v_{n, 0}^{\prime d} \Lambda_{D}\right)$;

Obtain $\boldsymbol{\pi}^{\prime}(n, 1), 0 \leq n \leq C$ using (16), (19) and (13), (14);

if $\left(\alpha_{U_{S}^{\prime}}>0\right)$ add_state $\left(s_{0,1}^{\prime \prime}, \alpha_{U_{S}^{\prime}}\right) ; L=1$;

$\operatorname{do}\{$

for (each $\left.n, L-1 \leq n \leq L+C-1: \boldsymbol{\pi}^{\prime}(n, L)^{U_{S}^{\prime}} \neq 0\right)\{$

Compute probabilities $w_{n, L}^{\prime u u}, w_{n, L}^{\prime u d}, q_{n, L}^{\prime u}$; if (absorb) Compute probability $v_{n, L}^{\prime \prime}$;

if $\left(w_{n, L}^{\prime u u}>0\right)\left\{\right.$ add_state $\left(s_{n+1, L+1}^{\prime u}, 0\right) ;$ add_transition $\left.\left(s_{n, L}^{\prime u}, s_{n+1, L+1}^{\prime u}, w_{n, L}^{\prime u u} \Lambda_{U}\right) ;\right\}$

if $\left(w_{n, L}^{\text {,ud }}>0\right)$

if $(n+1 \leq L+C-1)\{$

\}

if $\left(s_{n+1, L}^{\prime d}\right.$ not in $\left.V\right)$ add_state $\left(s_{n+1, L}^{\prime d}, 0\right) ;$ add_transition $\left(s_{n, L}^{\prime u}, s_{n+1, L}^{\prime d}, w_{n, L}^{\prime \prime d} \Lambda_{U}\right)$;

else add_transition $\left(s_{n, L}^{\prime u}, a, w_{n, L}^{\left.\text {rud } \Lambda_{U}\right)}\right.$;

if $\left(q_{n, L}^{\prime u}>0\right)$

if $\left(r \in U_{S}\right)$ add_transition $\left(s_{n, L}^{\prime u}, s_{0,1}^{u}, q_{n, L}^{\prime u} \Lambda_{U}\right)$; else add_transition $\left(s_{n, L}^{\prime u}, s_{0,0}^{d}, q_{n, L}^{\prime u} \Lambda_{U}\right)$;

\}

if $\left(\right.$ absorb \&\& $\left.v_{n, L}^{\prime u}>0\right)$ add_transition $\left(s_{n, L}^{\prime \prime}, f, v_{n, L}^{\prime \prime} \Lambda_{U}\right)$;

for (each $\left.n, L-1 \leq n \leq L+C-1: \boldsymbol{\pi}^{\prime}(n, L)^{D_{S}^{\prime}} \neq \mathbf{0}\right)\{$

Compute probabilities $w_{n, L}^{\prime d u}, w_{n, L}^{\prime d d}, q_{n, L}^{\prime d} ;$ if (absorb) Compute probability $v_{n, L}^{\prime d}$;

if $\left(w_{n, L}^{\prime d u}>0\right)\{$ \}

if $\left(s_{n+1, L+1}^{\prime u}\right.$ not in $\left.V\right)$ add_state $\left(s_{n+1, L+1}^{\prime \prime}, 0\right) ;$ add_transition $\left(s_{n, L}^{\prime d}, s_{n+1, L+1}^{\prime u}, w_{n, L}^{\prime d u} \Lambda_{D}\right)$;

if $\left(w_{n, L}^{\prime d d}>0\right)$

if $(n+1 \leq L+C-1)\{$ \}

if $\left(s_{n+1, L}^{\prime d}\right.$ not in $\left.V\right)$ add_state $\left(s_{n+1, L}^{\prime d}, 0\right) ;$ add_transition $\left(s_{n, L}^{\prime d}, s_{n+1, L}^{\prime d}, w_{n, L}^{\prime d d} \Lambda_{D}\right)$;

else add_transition $\left(s_{n, L}^{\prime d}, a, w_{n, L}^{\prime d d} \Lambda_{D}\right)$;

if $\left(q_{n, K}^{\prime d}>0\right)$

if $\left(r \in U_{S}\right)$ add_transition $\left(s_{n, L}^{\prime d}, s_{0,1}^{u}, q_{n, L}^{\prime d} \Lambda_{D}\right)$; else add_transition $\left(s_{n, L}^{\prime d}, s_{0,0}^{d}, q_{n, K}^{\prime d} \Lambda_{D}\right)$; \}

if $\left(\right.$ absorb \&\& $\left.v_{n, L}^{\prime d}>0\right)$ add_transition $\left(s_{n, L}^{\prime d}, f, v_{n, L}^{\prime d} \Lambda_{D}\right)$;

\}

$L++$; Obtain $\pi^{\prime}(n, L), L-1 \leq n \leq L+C-1$ using (13), (14), (20);

$a_{C}^{\prime}=\sum_{n=L-1}^{L+C-1} \sum_{i \in S^{\prime}} \pi_{i}^{\prime}(n, L)$;

until $\left(a_{C}^{\prime} \sum_{k=L}^{\infty} e^{-\Lambda_{U} t_{\max }}\left(\Lambda_{U} t_{\max }\right)^{k} / k ! \leq \epsilon / 8\right)$;

for (each $n, L-1 \leq n \leq L+C-1: s_{n, L}^{\prime \prime}$ in $V$ ) add_transition $\left(s_{n, L}^{\prime \prime}, b, \Lambda_{U}\right)$;

\}

for (each $n, L-1 \leq n \leq L+C-1: s_{n, L}^{\prime d}$ in $V$ ) add_transition $\left(s_{n, L}^{\prime d}, b, \Lambda_{D}\right)$;

\}

Compute $\widetilde{\operatorname{IAVCD}}\left(t_{1}, p_{1}\right), \widetilde{\operatorname{IAVCD}}\left(t_{2}, p_{2}\right), \ldots, \widetilde{\operatorname{IAVCD}}\left(t_{n}, p_{n}\right)$ as the interval availability complementary distribution of $V$ with subset of up states including the states $s_{n, k}^{u}, s_{n, k}^{\prime u}$, and state $f$, if absorb and $f \in U$, using Algorithm A of [14] with error $\leq \epsilon / 2$;

Fig. 8. Algorithmic description of the method (continuation). 
$s_{n, k}^{\prime u}, s_{n, k}^{\prime d}$ are added in a similar way, with the truncation error associated with the parameter $L$ being controlled for $t=t_{\max }$, since the model error truncation upper bound associated with $L$ also increases with $t$. The particular case $U_{S}^{\prime}=\emptyset$ receives a special treatment. In that case, besides state $a$ and state $f$, if $\Omega=S \cup\{f\}$, the truncated transformed model includes the state $s_{0,1}^{u}$, if $r \in U_{S}$, the state $s_{0,0}^{d}$, if $r \in D_{S}$, the states $s_{1,1}^{d}, \ldots, s_{C, 1}^{d}$, if $r \in U_{S}$ and $\lambda_{r, D_{S}^{\prime}}>0$, the states $s_{1,0}^{d}, \ldots, s_{C-1,0}^{d}$, if $r \in D_{S}$ and $\lambda_{r, D_{S}^{\prime}}>0$, and the states $s_{0,0}^{\prime d}, s_{1,0}^{\prime d}, \ldots, s_{C-1,0}^{\prime d}$, if $\alpha_{S^{\prime}}>0$. Besides the storage required to hold the transition probability matrix $\mathbf{P}$ of the randomized DTMC, the storage requirements of Algorithm A of [6] are basically $C^{\prime}+2$ vectors of size $|\Omega|$, necessary to compute recurrently the vectors $\mathbf{Y}_{n, k}$. Regarding our method, in the case $U_{S}^{\prime} \neq \emptyset$, vectors $\pi(n, k)$ and $\pi^{\prime}(n, k)$ can be computed over $C+3$ vectors of size $|S|$ and $C$ will have a value typically identical to the value of the truncation parameter $C^{\prime}$ of Algorithm A of [6]. Then, in the case $U_{S}^{\prime} \neq \emptyset$, the storage overhead of our method, during its first phase, with respect to Algorithm A of [6] includes approximately three vectors of size $|S|$ (our method needs two vectors of size $|S|$ to hold $P_{i, U_{S}^{\prime}}$ and $P_{i, D_{S}^{\prime}}, i \in S$ ) and the storage required to hold the truncated transformed model. In the case $U_{S}^{\prime}=\emptyset$, the required vectors $\pi(n, k)$ and $\pi^{\prime}(n, k)$ can be computed over two vectors of size $|S|$ and the storage requirements of our method in its first phase will tend to be smaller than the storage requirements of Algorithm A of [6]. In the second phase, the storage consumption of our method can be smaller or greater than the storage consumption of Algorithm A of [6], depending basically on the relative sizes of the truncated transformed model and $X$.

The method requires the computation of Poisson probabilities $\mathrm{e}^{-\lambda} \lambda^{k} / k$ !. Our implementation uses the method described in [15, pp. 1028-1029], which has good numerical stability. Finally, the method requires the computation of summations of the form $S_{\lambda}(m)=\sum_{k=m+1}^{\infty} \mathrm{e}^{-\lambda} \lambda^{k} / k !$ and $S_{\lambda}^{\prime \prime \prime}(m)=\sum_{k=m}^{\infty}$ $(k-m+2) \mathrm{e}^{-\lambda} \lambda^{k} / k$ ! for increasing values of $m$. An efficient and numerically stable procedure to compute $S_{\lambda}(m)$ for increasing values of $m$ which can be embedded in the method is described in [13]. It is also described in [13] an efficient and numerically stable procedure to compute $S_{\lambda}^{\prime}(m)=\sum_{k=m+1}^{\infty}(k-m) \mathrm{e}^{-\lambda} \lambda^{k} / k$ ! for increasing values of $m$; that procedure can easily be adapted to obtain an efficient and numerically stable procedure to compute $S_{\lambda}^{\prime \prime \prime}(m)$ for increasing values of $m$ which can be embedded in the method.

\section{Theoretical properties}

A salient feature of the method proposed in this paper is that, for the case $U_{S}^{\prime} \neq \emptyset$, the truncation parameters $K$ and $L$ are smooth functions of $t$ :

Theorem 4. Assume $U_{S}^{\prime} \neq \emptyset$. For the case $\alpha_{S^{\prime}}>0$, the truncation parameters $K$ and $L$ are $O\left(\log \left(\Lambda_{U} t / \varepsilon\right)\right)$. For the case $\alpha_{S}^{\prime}=0$, the truncation parameter $K$ is $O\left(\log \left(\Lambda_{U} t / \varepsilon\right)\right)$.

Proof. See the Appendix A.

Consider the case $U_{S}^{\prime} \neq \emptyset$. Then, the fact that, as asserted by Theorem 4 , the truncation parameters $K$ and $L$ are smooth functions of $t$ is called benign behavior and implies that for large enough models and large enough $t$, the proposed method will be significantly faster than Algorithm A of [6] (and all other previously proposed general methods). To see this, note that, for not too small models, the 
computational cost (number of floating point operations) of the first phase of the proposed method is, for the case $\alpha_{S^{\prime}}>0$, roughly the sum of a component proportional to $K$ and $C$ and a component proportional to $L$ and $C$, and is, for the case $\alpha_{S^{\prime}}=0$, roughly proportional to $K$ and $C$, while the computational cost of Algorithm A of [6] is roughly proportional to the truncation parameters $N$ and $C^{\prime}$ of that method, where $N$ is approximately proportional to $t$ for large $t$ and $C^{\prime} \approx C$. This fact, with Theorem 4, implies that, for not too small models and large enough $t$, the computational cost of the first phase of the proposed method will be significantly smaller than the computational cost of Algorithm A of [6]. Since the maximum output rate of the truncated transformed model is $1+\theta$ times the maximum output rate of $X$, where $\theta$ is very small, the truncation parameters of Algorithm A of [6] applied to the solution of the truncated transformed model will be approximately equal to the truncation parameters of that method applied to the solution of the original model $X$ and, then, the ratio between the computational cost of the second phase of the proposed method and the computational cost of Algorithm A of [6] will be roughly equal to the ratio between the number of transitions of the randomized DTMC of the truncated transformed model with randomization rate $\max \left\{\Lambda_{U}, \Lambda_{D}\right\}$ and the number of transitions of the randomized DTMC of $X$ with randomization rate $\max _{i \in S} \lambda_{i}$, implying that the computational cost of the second phase of the proposed method will be significantly smaller than the computational cost of Algorithm A of [6] for large enough $X$.

For the case $U_{S}^{\prime}=\emptyset$, the truncated transformed model has at most $2 C+3$ states, and it is clear that for large enough models and large enough $t$, the proposed method will be significantly faster than Algorithm A of [6] (and all other previously proposed general methods).

The performance of the proposed method depends on the selection of the regenerative state $r$, since that selection influences the behavior of $a_{C}(n)$ and $a_{C}^{\prime}(n)$. Ideally, state $r$ should be selected so that $a_{C}(n)$ and $a_{C}^{\prime}(n)$ decrease as fast as possible. For as wide class of models as covered by the method, automatic selection of $r$ does not seem to be easy in general, and, then, the method relies on the user's intuition to make an appropriate selection. We will consider next a class of models $\mathrm{C}_{1}$ for which a natural selection for the regenerative state exists, and, for models in that class and that natural selection, will obtain stronger theoretical results than the benign behavior asserted by Theorem 4 assessing the performance of the method in terms of "visible" model characteristics.

The model class $\mathrm{C}_{1}$ includes all CTMCs $X$ with the properties described in Section 1 and $\left|U_{S}\right| \geqslant 2^{5}$ for which a partition $U_{0} \cup U_{1} \cup \cdots \cup U_{N_{C}}$ for $U_{S}$ exists satisfying the following two properties:

P1. $U_{0}=\{o\}$ (i.e. $\left.\left|U_{0}\right|=1\right)$.

P2. $\max _{0 \leqslant k \leqslant N_{C}} \max _{i \in U_{k}} \lambda_{i, U_{k}-\{i\} \cup U_{k+1} \cup \cdots \cup U_{N_{C}} \cup D_{S}}$ is significantly smaller than $\min _{0<k \leqslant N_{C}} \min _{i \in U_{k}} \lambda_{i, U_{0} \cup \ldots \cup U_{k-1} \cup\{f\}}>0$.

Class $\mathrm{C}_{1}$ includes failure/repair models of coherent fault-tolerant systems with exponential failure and repair time distributions and repair in every state with failed components when failure rates are significantly smaller than repair rates (the typical case). For those models, a partition for which properties P1 and P2 are satisfied is $U_{k}=\{$ up states with $k$ failed components $\}$. Models of non-coherent

\footnotetext{
${ }^{5}$ In the case $\left|U_{S}\right|=1$, the selection $r=o$ makes $U_{S}^{\prime}=\emptyset$, and the number of states of the truncated transformed model would be 2 plus 1 , if $\Omega=S \cup\{f\}$, plus $C$, if $\lambda_{r, D_{S}^{\prime}}>0$, plus $C$, if $\alpha_{S^{\prime}}>0$, and the performance of the method is also easy to predict.
} 
systems may not belong to class $\mathrm{C}_{1}$, because in those models there may be fast "repair" transitions taking the system down and, thus, going from $U_{S}$ to $D_{S}$. Repair times with acyclic phase-type distributions [16] (which can be used to fit distributions of non-exponential positive random variables [17]) can, however, be allowed.

Since, for class $\mathrm{C}_{1}$ models, $X$ moves "fast" to state $o$ or, if existent, to state $f$, a natural selection for the regenerative state for those models is $r=o$. Consider a class $\mathrm{C}_{1}$ model and a partition $U_{0} \cup \cdots \cup U_{N_{C}}$ for $U_{S}$ satisfying properties $\mathrm{P} 1$ and $\mathrm{P} 2$, and let

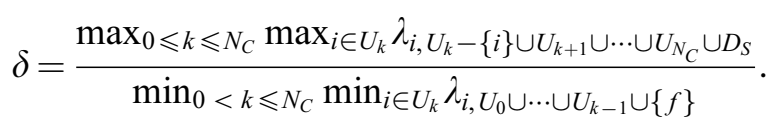

The parameter $\delta$ can be seen as a "rarity" parameter measuring how strongly property P2 is satisfied. In terms of the rarity parameter $\delta$, we can model the transition rates from $i \in U_{k}$ to $j \in U_{k}-\{i\} \cup$ $U_{k+1} \cup \cdots \cup U_{N_{C}} \cup D_{S}, 0 \leqslant k \leqslant N_{C}$ as $\lambda_{i, j}=\Lambda_{i, j} \delta$, where $\Lambda_{i, j}$ are constants, and the remaining transition rates as constants, and study the behavior of the method with the selection $r=o$ as $\delta \rightarrow 0$. Let $P_{i, j}(\delta)$ denote the transition probabilities of $\hat{X}$ as a function of the rarity parameter $\delta$. Note that, for $i \in U_{k}, j \in U_{k}-\{i\} \cup U_{k+1} \cup \cdots \cup U_{N_{C}} \cup D_{S}, 0 \leqslant k \leqslant N_{C}, \lim _{\delta \rightarrow 0} P_{i, j}(\delta)=0$ and that

$$
\lim _{\delta \rightarrow 0} P_{i, i}(\delta)=1-\frac{\lambda_{i, U_{0} \cup \cdots \cup U_{k-1} \cup\{f\}}}{(1+\theta) \max _{0 \leqslant k \leqslant N_{C}} \max _{i \in U_{k}} \lambda_{i, U_{0} \cup \cdots \cup U_{k-1} \cup\{f\}}}, i \in U_{k}, 0<k \leqslant N_{C} .
$$

Let $\mathbf{P}(\delta)$ denote the transition probability matrix of $\hat{X}$ as a function of $\delta$, let $\mathbf{P}_{U_{S}, U_{S}}^{\prime}(\delta)$ be the matrix obtained from $\mathbf{P}_{U_{S}, U_{S}}(\delta)$ by making null the entries $(i, r), i \in U_{S}$ and let $\mathbf{P}_{1}(\delta)=\mathbf{P}_{U_{S}, U_{S}}^{\prime}(\delta)+$ $\mathbf{P}_{U_{S}, D_{S}}(\delta) \mathbf{P}_{D_{S}, D_{S}}^{-1} \mathbf{P}_{D_{S}, U_{S}}, \mathbf{P}_{2}(\delta)=\mathbf{P}_{U_{S}^{\prime}, U_{S}^{\prime}}(\delta)+\mathbf{P}_{U_{S}^{\prime}, D_{S}}(\delta) \mathbf{P}_{D_{S}, D_{S}}^{-1} \mathbf{P}_{D_{S}, U_{S}^{\prime}}$. Note that the submatrices $\mathbf{P}_{D_{S}, D_{S}}$, $\mathbf{P}_{D_{S}, U_{S}}$ and $\mathbf{P}_{D_{S}, U_{S}^{\prime}}$ of $\mathbf{P}(\delta)$ do not depend on $\delta$ and that, being $\mathbf{P}_{D_{S}, D_{S}}$ a diagonal submatrix of the restriction to $S$ of the transition probability matrix of $Z$ and being all states in $S$ transient in $Z, \mathbf{P}_{D_{S}, D_{S}}^{-1}$ exists. Then, denoting by $\rho(\mathbf{A})$ the spectral radius of matrix $\mathbf{A}$, we have the following result: ${ }^{6}$

Theorem 5. For class $\mathrm{C}_{1}$ models and the selection $r=o, a_{C}(n) \leqslant(C+1) h(n)$ and $a_{C}^{\prime}(n) \leqslant$ $\alpha_{S^{\prime}}(C+1) h^{\prime}(n)$, where, for $n \rightarrow \infty, h(n) \sim B(\delta)\left(\begin{array}{c}n-1 \\ p(\delta)-1\end{array}\right) \rho\left(\mathbf{P}_{1}(\delta)^{\mathrm{T}}\right)^{n}, B(\delta)>0, p(\delta)$ integer $\geqslant 1$ and $h^{\prime}(n) \sim B^{\prime}(\delta)\left(\begin{array}{c}n-1 \\ p^{\prime}(\delta)-1\end{array}\right) \rho\left(\mathbf{P}_{2}(\delta)^{\mathrm{T}}\right)^{n}, B^{\prime}(\delta)>0, \quad p^{\prime}(\delta)$ integer $\geqslant 1$, with $\lim _{\delta \rightarrow 0} \rho\left(\mathbf{P}_{1}(\delta)^{\mathrm{T}}\right)$ $=\lim _{\delta \rightarrow 0} \rho\left(\mathbf{P}_{2}(\delta)^{\mathrm{T}}\right)=\rho$,

$$
\rho=1-\frac{\min _{0<k \leqslant N_{C}} \min _{i \in U_{k}} \lambda_{i, U_{0} \cup \cdots \cup U_{k-1} \cup\{f\}}}{(1+\theta) \max _{0 \leqslant k} \max _{i \in U_{k}} \lambda_{i, U_{0} \cup \cdots \cup U_{k-1} \cup\{f\}}} .
$$

Proof. Let $b(k)$ be the probability that $Z$ has made $k$ or more visits to $U_{S}$. We have, for $n \geqslant 0$ and $0 \leqslant k \leqslant n+1$,

$$
\sum_{i \in S} \pi_{i}(n, k)=\sum_{i \in S} P\left[Z_{n}=i \wedge \#\left(Z_{0: n} \in U_{S}\right)=k\right] \leqslant P\left[\#\left(Z_{0: n} \in U_{S}\right)=k\right] \leqslant b(k)
$$

\footnotetext{
${ }^{6} x(k) \sim y(k)$ for $k \rightarrow \infty$ denotes $\lim _{k \rightarrow \infty} x(k) / y(k)=1$
} 
and

$$
a_{C}(k)=\sum_{n=k-1}^{k+C-1} \sum_{i \in S} \pi_{i}(n, k) \leqslant \sum_{n=k-1}^{k+C-1} b(k)=(C+1) b(k) .
$$

Let $q_{i}(n), i \in U_{S}$ be the probability that $Z$ has made $n$ or more visits to $U_{S}$ and that the $n$th of such visits has been to state $i$. Trivially, $b(n)=\sum_{i \in U_{S}} q_{i}(n)$. Let $\mathbf{q}(n)$ be the row vector $\left(q_{i}(n)\right)_{i \in U_{S}}$. Taking into account $Z_{0}=r \in U_{S}$, we have $\mathbf{q}(1)=\left(I_{i=r}\right)_{i \in U_{S}}$ and $\left\|\mathbf{q}(1)^{\mathrm{T}}\right\|_{1}=1$. Also, from the definition of $\mathbf{P}_{1}(\delta), \mathbf{q}(n)^{\mathrm{T}}=\left(\mathbf{P}_{1}(\delta)^{\mathrm{T}}\right)^{n-1} \mathbf{q}(1)^{\mathrm{T}}$. Then,

$$
b(n)=\left\|\mathbf{q}(n)^{\mathrm{T}}\right\|_{1} \leqslant\left\|\left(\mathbf{P}_{1}(\delta)^{\mathrm{T}}\right)^{n-1}\right\|_{1}\left\|\mathbf{q}(1)^{\mathrm{T}}\right\|_{1}=\left\|\left(\mathbf{P}_{1}(\delta)^{\mathrm{T}}\right)^{n-1}\right\|_{1}=h(n) .
$$

We have [18, Theorem 3.1] that, for $n \rightarrow \infty,\left\|\left(\mathbf{P}_{1}(\delta)^{\mathrm{T}}\right)^{n-1}\right\|_{1} \sim B(\delta)\left(\begin{array}{c}n-1 \\ p(\delta)-1\end{array}\right) \rho\left(\mathbf{P}_{1}(\delta)^{\mathrm{T}}\right)^{n}, B(\delta)>0$, $p(\delta)$ integer $\geqslant 1$. Also, since the eigenvalues of a matrix are continuous functions of the elements of the matrix [19, Theorem 3.13], $\lim _{\delta \rightarrow 0} \rho\left(\mathbf{P}_{1}(\delta)^{\mathrm{T}}\right)=\rho\left(\mathbf{P}_{1}(0)^{\mathrm{T}}\right)$. But $\mathbf{P}_{U_{S}, D_{S}}(0)=\mathbf{0}$, where $\mathbf{0}$ is a matrix of zeroes, and, then, $\mathbf{P}_{1}(0)^{\mathrm{T}}=\mathbf{P}_{U_{S}, U_{S}}^{\prime}(0)^{\mathrm{T}}$. Also, with the ordering of states $U_{0}, U_{1}, \ldots, U_{N_{C}}$, the elements in the lower triangular portion of $\mathbf{P}_{U_{S}, U_{S}}^{\prime}(0)^{\mathrm{T}}$ are 0 and the diagonal elements have values 0 and values $1-\lambda_{i, U_{0} \cup \ldots \cup U_{k-1} \cup\{f\}} /\left((1+\theta) \max _{0 \leqslant k \leqslant N_{C}} \max _{i \in U_{k}} \lambda_{i, U_{0} \cup \ldots \cup U_{k-1} \cup\{f\}}\right), i \in U_{k}, 0<k \leqslant N_{C}$ and, then, $\rho\left(\mathbf{P}_{1}(0)^{\mathrm{T}}\right)=\rho\left(\mathbf{P}_{U_{S}, U_{S}}^{\prime}(0)^{\mathrm{T}}\right)=\rho$.

Let $b^{\prime}(k)$ be the probability that $Z^{\prime}$ has made $k$ or more visits to $U_{S}^{\prime}$. We have, for $n \geqslant 0$ and $0 \leqslant k \leqslant n+1$,

$$
\sum_{i \in S^{\prime}} \pi_{i}^{\prime}(n, k)=\sum_{i \in S^{\prime}} P\left[Z_{n}^{\prime}=i \wedge \#\left(Z_{0: n}^{\prime} \in U_{S}^{\prime}\right)=k\right] \leqslant P\left[\#\left(Z_{0: n}^{\prime} \in U_{S}^{\prime}\right)=k\right] \leqslant b^{\prime}(k)
$$

and

$$
a_{C}^{\prime}(k)=\sum_{n=k-1}^{k+C-1} \sum_{i \in S^{\prime}} \pi_{i}^{\prime}(n, k) \leqslant \sum_{n=k-1}^{k+C-1} b^{\prime}(k)=(C+1) b^{\prime}(k) .
$$

Let $q_{i}^{\prime}(n), i \in U_{S}^{\prime}$ be the probability that $Z^{\prime}$ has made $n$ or more visits to $U_{S}^{\prime}$ and that the $n$th of such visits has been to state $i$. Trivially, $b^{\prime}(n)=\sum_{i \in U_{S}^{\prime}} q_{i}^{\prime}(n)$. Let $\mathbf{q}^{\prime}(n)$ be the row vector $\left(q_{i}^{\prime}(n)\right)_{i \in U_{S}^{\prime}}$. Taking into account $P\left[Z_{0}^{\prime} \in S^{\prime}\right]=\alpha_{S^{\prime}}$ and that the states in $S^{\prime}$ are transient in $Z^{\prime}$, we have $\left\|\mathbf{q}^{\prime}(1)^{\mathrm{T}}\right\|_{1} \leqslant \alpha_{S^{\prime}}$. Also, from the definition of $\mathbf{P}_{2}(\delta), \mathbf{q}^{\prime}(n)^{\mathrm{T}}=\left(\mathbf{P}_{2}(\delta)^{\mathrm{T}}\right)^{n-1} \mathbf{q}^{\prime}(1)^{\mathrm{T}}$. Then,

$$
b^{\prime}(n)=\left\|\mathbf{q}^{\prime}(n)^{\mathrm{T}}\right\|_{1} \leqslant\left\|\left(\mathbf{P}_{2}(\delta)^{\mathrm{T}}\right)^{n-1}\right\|_{1}\left\|\mathbf{q}^{\prime}(1)^{\mathrm{T}}\right\|_{1} \leqslant \alpha_{S^{\prime}}\left\|\left(\mathbf{P}_{2}(\delta)^{\mathrm{T}}\right)^{n-1}\right\|_{1}=\alpha_{S^{\prime}} h^{\prime}(n),
$$

with $h^{\prime}(n)=\left\|\left(\mathbf{P}_{2}(\delta)^{\mathrm{T}}\right)^{n-1}\right\|_{1}$. As before, for $n \rightarrow \infty,\left\|\left(\mathbf{P}_{2}(\delta)^{\mathrm{T}}\right)^{n-1}\right\|_{1} \sim B^{\prime}(\delta)\left(\begin{array}{c}n-1 \\ p^{\prime}(\delta)-1\end{array}\right) \rho\left(\mathbf{P}_{2}(\delta)^{\mathrm{T}}\right)^{n}$, $B^{\prime}(\delta)>0, p^{\prime}(\delta)$ integer $\geqslant 1$, and $\lim _{\delta \rightarrow 0} \rho\left(\mathbf{P}_{2}(\delta)^{\mathrm{T}}\right)=\rho\left(\mathbf{P}_{2}(0)^{\mathrm{T}}\right)$. Since $\mathbf{P}_{U_{S}^{\prime}, D_{S}}(0)=\mathbf{0}, \mathbf{P}_{2}(0)^{\mathrm{T}}=$ $\mathbf{P}_{U_{S}^{\prime}, U_{S}^{\prime}}(0)^{\mathrm{T}}$. But, with the ordering of states $U_{1}, U_{2}, \ldots, U_{N_{C}}$, the elements in the lower triangular portion of $\mathbf{P}_{U_{S}^{\prime}, U_{S}^{\prime}}(0)^{\mathrm{T}}$ are 0 and the diagonal elements have values $1-\lambda_{i, U_{0} \cup \cdots \cup U_{k-1} \cup\{f\}} /((1+\theta)$ $\left.\max _{0 \leqslant k \leqslant N_{C}} \max _{i \in U_{k}} \lambda_{i, U_{0} \cup \cdots \cup U_{k-1} \cup\{f\}}\right), i \in U_{k}, 0<k \leqslant N_{C}$ and, then, $\rho\left(\mathbf{P}_{2}(0)^{\mathrm{T}}\right)=\rho\left(\mathbf{P}_{U_{S}^{\prime}, U_{S}^{\prime}}(0)^{\mathrm{T}}\right)=\rho$.

According to Theorem 5, both $a_{C}(n)$ and $a_{C}^{\prime}(n)$ decay asymptotically by a factor $\rho$. For class $\mathrm{C}_{1}$ models and the selection $r=o$, because of property $\mathrm{P} 2, \min _{0<k \leqslant N_{C}} \min _{i \in U_{k}} \lambda_{i, U_{0} \cup \ldots \cup U_{k-1} \cup\{f\}} \approx$ 
$\min _{i \in U_{S}^{\prime}} \lambda_{i}$ and, taking into account $\theta \ll 1,(1+\theta) \max _{0 \leqslant k \leqslant N_{C}} \max _{i \in U_{K}} \lambda_{i, U_{0} \cup \ldots \cup U_{k-1} \cup\{f\}} \approx \max _{i \in U_{S}} \lambda_{i}$. Then, letting $R=\max _{i \in U_{S}} \lambda_{i} / \min _{i \in U_{S}^{\prime}} \lambda_{i}$, we have $\rho \approx 1-1 / R$, and the values of the truncation parameters $K$ and $L$ will be mainly determined by $R$ : the smaller $R$, the smaller $K$ and $L$. In fact, we can roughly approximate $h(n)$ and $h^{\prime}(n)$ by $(1-1 / R)^{n}$ and roughly approximate $a_{C}(n)$ by $h(n)$ and $a_{C}^{\prime}(n)$ by $h^{\prime}(n)$ and, then, it is easy to show that, for $R \gg 1, K$ and $L$ would be proportional to $R$. As a rule of thumb (see [13]), $K$ and $L$ can be roughly upper bounded by $30 R$. We note that $R$ is a "visible" characteristic of the model (one that can be easily computed/estimated). Then, since the truncation parameter $C$ of our method and the truncation parameters of Algorithm A of [6] can be easily computed, the previous rule of thumb can be used in practice to anticipate when our method can be expected to be significantly faster than Algorithm A of [6] for class $C_{1}$ models with the selection $r=o$.

\section{Numerical analysis}

In this section we will illustrate the application of the method developed in this paper and compare it with algorithm $A$ of [6] using a parametric example belonging to class $\mathrm{C}_{1}$. The example is the fault-tolerant database system whose block diagram for the case 4 disk sets is given in Fig. 9. The system is made up of two processing subsystems, each including one processor $\mathrm{P}$ and two memories $\mathrm{M}$, a set of controllers with two controllers, and $D$ sets of disks, each with four disks. The system is operational if at least one processor and one memory connected to it are operational, one controller is operational, and three disks of each set are operational. Processors fail with rate $\lambda_{\mathrm{P}}=2 \times 10^{-5} \mathrm{~h}^{-1}$; a processor failure is soft with probability $S_{\mathrm{P}}=0.8$ and hard with probability $1-S_{\mathrm{P}}=0.2$. Memories fail with rate $\lambda_{\mathrm{M}}=2 \times 10^{-4} \mathrm{~h}^{-1}$. Controllers fail with rate $\lambda_{\mathrm{C}}=2 \times 10^{-4} \mathrm{~h}^{-1}$. Disks fail with rates $\lambda_{\mathrm{D}}=3 \times 10^{-4} \mathrm{~h}^{-1}$. A failure of a controller is propagated to two disks of one set with probability $1-C_{\mathrm{C}}=0.10$. The disk set over which the failure is propagated is taken at random. Components do not fail when the system is down. There is an unlimited number of repairmen which perform restarts of processors in soft

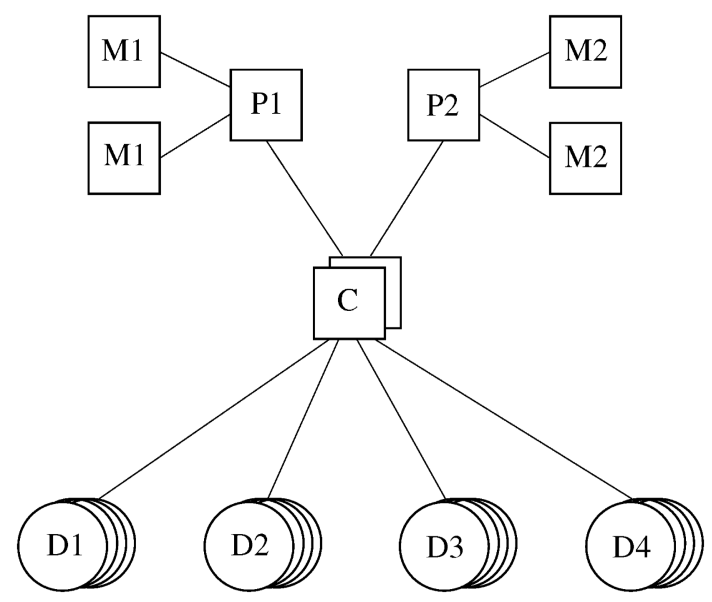

Fig. 9. Block diagram of the example with 4 disk sets. 
Table 1

Number of states, number of transitions and steady-state unavailability for the example as a function of $D$

\begin{tabular}{lrrr}
\hline$D$ & \multicolumn{1}{c}{ States } & Transitions & UAV \\
\hline 4 & 8096 & 36,109 & $4.48218 \times 10^{-5}$ \\
8 & 211,456 & $1,364,173$ & $4.93011 \times 10^{-5}$ \\
\hline
\end{tabular}

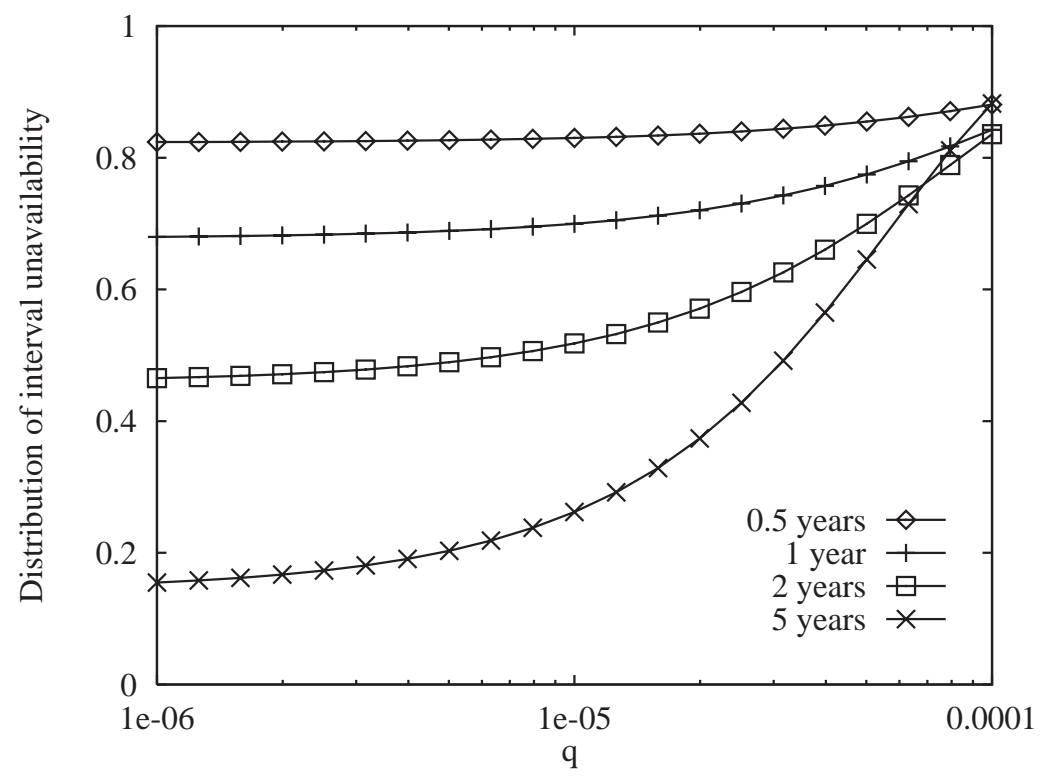

Fig. 10. $\operatorname{IAVCD}(t, 1-q)$ as a function of $q$ for several $t$ for the example with $D=4$ and initial state the state with all components unfailed.

failure with rate $\mu_{\mathrm{PS}}=0.5 \mathrm{~h}^{-1}$. The other repair actions are performed by another repairman with first priority given to disks, next to controllers, next to processors and next to memories. Components with the same repair priority are chosen at random. The repair rates are $\mu_{\mathrm{PH}}=0.2 \mathrm{~h}^{-1}$ for processors in hard failure mode, $\mu_{\mathrm{M}}=0.2 \mathrm{~h}^{-1}$ for memories, $\mu_{\mathrm{C}}=0.5 \mathrm{~h}^{-1}$ for controllers, and $\mu_{\mathrm{D}}=1 \mathrm{~h}^{-1}$ for disks. Table 1 gives the number of states and transitions of the model and the steady-state unavailability UAV for $D=4$ and 8 .

We start by analyzing the behavior of the interval availability complementary distribution. Fig. 10 plots $\operatorname{IAVCD}(t, 1-q)$ as a function of $q$, around the steady-state unavailability UAV $=4.48218 \times 10^{-5}$, for several values of $t(0.5,1,2$, and 5 years $)$, for the example with $D=4$ and initial state the state with all components unfailed. We can note how as $t$ increases $\operatorname{IAVCD}(t, 1-q)$ tends to its asymptotic shape ( 0 for $q<\mathrm{UAV}$ and 1 for $q>\mathrm{UAV}$ ). However, the asymptotic behavior is reached very slowly, which implies that the measure could be of interest for very large $t$. Since the computational effort of the algorithms to compute the distribution of the interval availability increases with $t$, this fact stresses the need for efficient algorithms. 


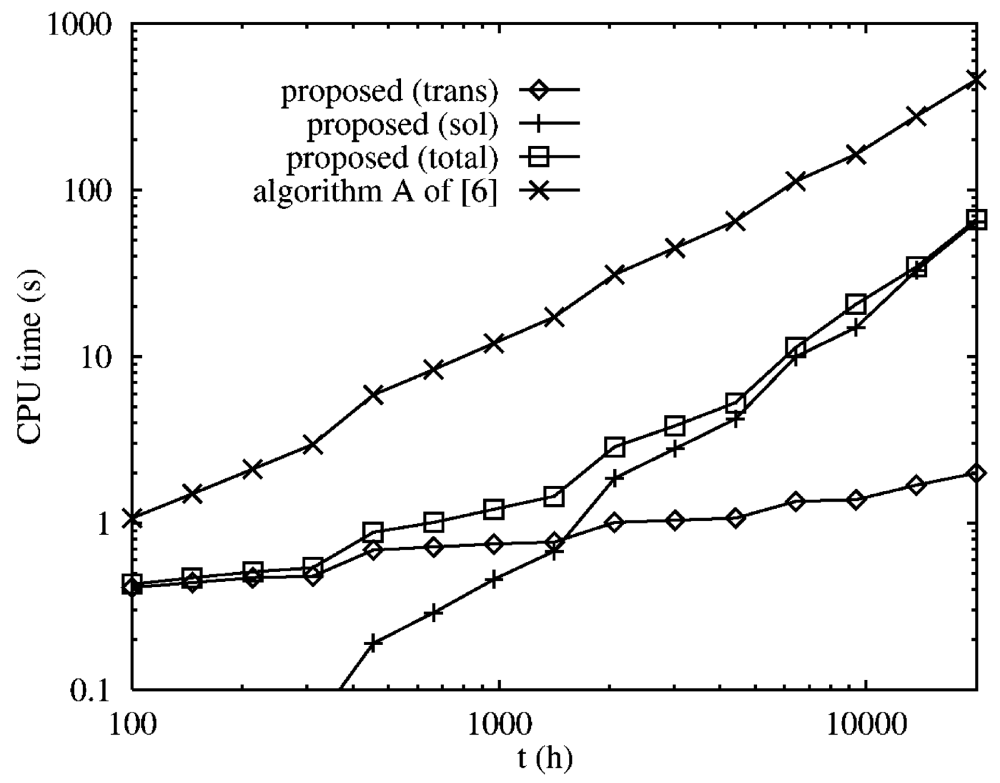

Fig. 11. CPU times in seconds required by the proposed method and Algorithm A of [6] for the computation of the distribution of the interval availability for the example with $D=4$ and $p=0.99999$ as a function of the mission time $t$ in hours.

We compare next, in terms of required CPU times, the method developed in this paper with algorithm $A$ of [6]. The example belongs to the class of models $C_{1}$ and, for our method, we will take the natural selection for the state $r$, i.e. we will take as regenerative state $r$ the state in which all components are unfailed. We will also assume that state to be the initial state of the model. All CPU times given here were obtained in a workstation with a Sun-Blade-1000 processor and 4 GB of memory. All the CPU times given for our method were measured. However, since for large $t$ and the large example $(D=8)$, the CPU times required by algorithm A of [6] are enormous, we estimated them using an approximate flop count for that method. All measurements or estimations were done for $\varepsilon=10^{-5}$. We give in Figs. 11-14 the CPU times of the first phase of our method (trans), the CPU times of the second phase (sol), the total CPU times of our method, and the CPU times of algorithm A of [6]. We take two values for $p(p=0.99999$ and $p=0.9999)$ and vary $t$ from 100 to $20,000 \mathrm{~h}$. Memory usage for the large example was about $65 \mathrm{MB}$. The required $C$ in our method increases as $t$ increases and $p$ gets smaller. The required $K$ is independent of $p$ and, according to Theorem 4, increases logarithmically with $t$. As an illustration of the dependence of $C$ on $t$ and $p$ and the dependence of $K$ on $t$, Table 2 gives the required values for $C$ and $K$ for the large example as a function of $t$ and, for the truncation parameter $C$, as a function of $p$. Regarding the values of $K$, we can note that the rule of thumb given in the previous section is rather pessimistic, since that rule predicts values of $K$ of $30 R \approx(30)(7.5)=225$, since $R$ is approximately equal to 7.5 for the example. That pessimism can be attributed to the fact that the required error, $\varepsilon$, is rather large $\left(\varepsilon=10^{-5}\right)$. In all cases, our method is faster. The speedups are smaller for the small example $(D=4)$ and larger for the large example $(D=8)$. For that large example and large $t$, the speedups 


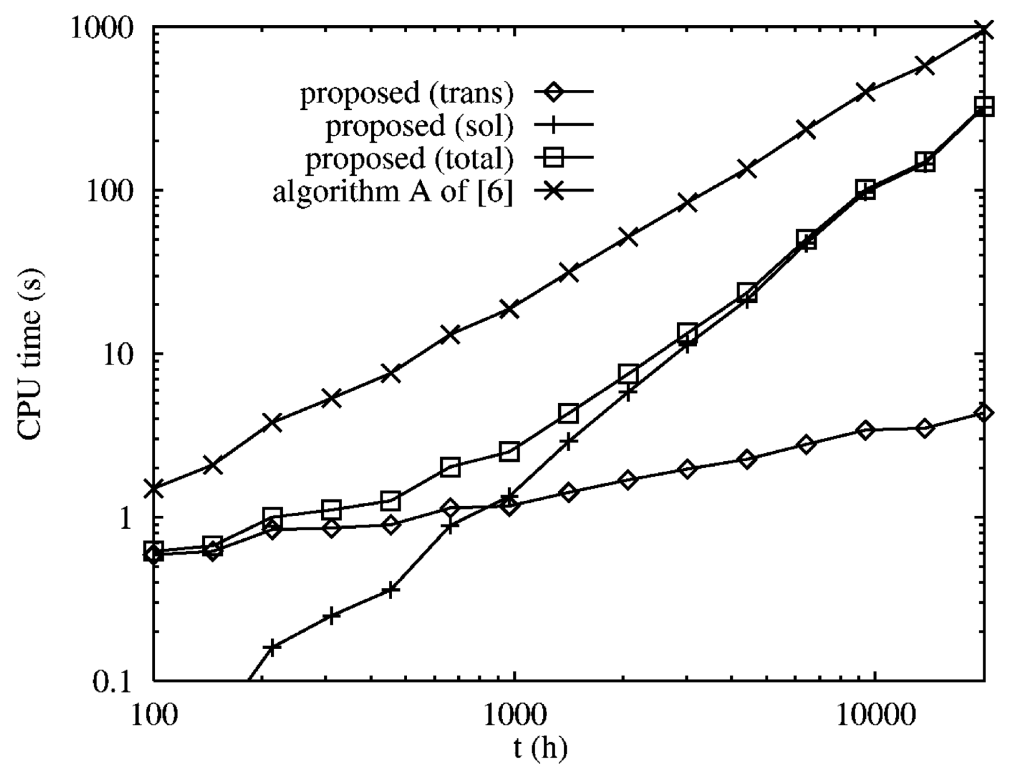

Fig. 12. CPU times in seconds required by the proposed method and Algorithm A of [6] for the computation of the distribution of the interval availability for the example with $D=4$ and $p=0.9999$ as a function of the mission time $t$ in hours.

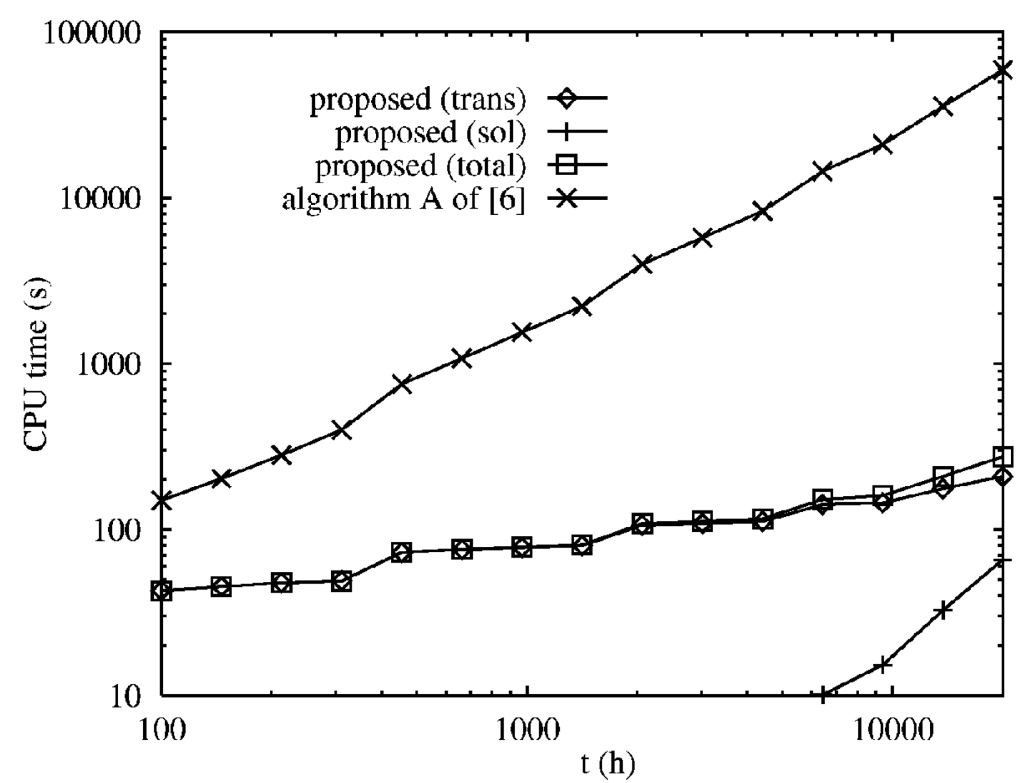

Fig. 13. CPU times in seconds required by the proposed method and Algorithm A of [6] for the computation of the distribution of the interval availability for the example with $D=8$ and $p=0.99999$ as a function of the mission time $t$ in hours. 


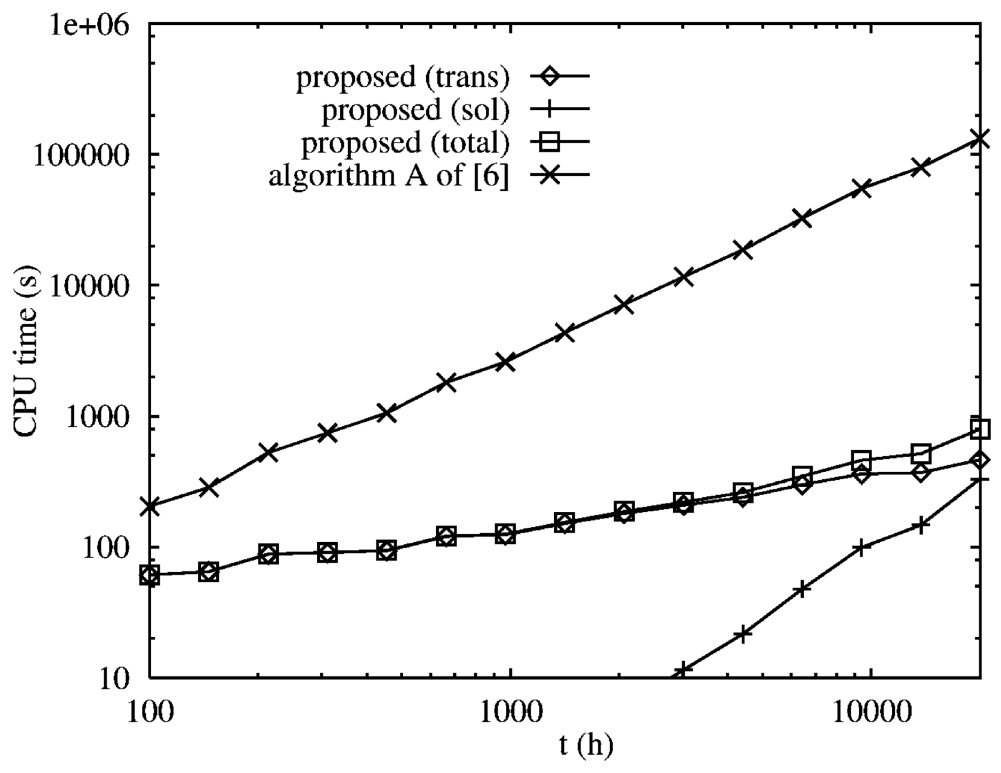

Fig. 14. CPU times in seconds required by the proposed method and Algorithm A of [6] for the computation of the distribution of the interval availability for the example with $D=8$ and $p=0.9999$ as a function of the mission time $t$ in hours.

Table 2

Required values of the truncation parameters $C$ and $K$ for the example with $D=8$ as a function of $t$ and $p$

\begin{tabular}{rrrr}
\hline & & & $C$ \\
\cline { 2 - 3 }$t(\mathrm{~h})$ & $K$ & $p=0.99999$ & $p=0.9999$ \\
\hline 100 & 78 & 2 & 3 \\
200 & 86 & 2 & 4 \\
500 & 94 & 3 & 4 \\
1000 & 100 & 3 & 5 \\
2000 & 105 & 4 & 7 \\
5000 & 112 & 4 & 10 \\
10,000 & 117 & 5 & 13 \\
20,000 & 123 & 7 & 16 \\
\hline
\end{tabular}

are important (for $t=20,000 \mathrm{~h}$, the speedup is 215 for $p=0.99999$ and 166 for $p=0.9999$ ). The speedups have practical significance, since algorithm A of [6] takes for $t=20,000 \mathrm{~h}$ more than $16 \mathrm{~h}$ for $p=0.99999$ and more than $36 \mathrm{~h}$ for $p=0.9999$, whereas our method only takes about $4 \mathrm{~min}$ for $p=0.99999$ and about $13 \mathrm{~min}$ for $p=0.9999$. It is also interesting to note that for small $t$ the CPU time of our method is dominated by the CPU time of the first phase, whereas for larger $t$ the second phase becomes also important and can even dominate the total CPU time when the model is of moderate size $(D=4)$. 


\section{Conclusions}

We have developed a new method for the computation of the distribution of the interval availability of fault-tolerant systems modeled by CTMCs which is based on a model transformation approach. Like previously proposed randomization-based methods, the new method is numerically stable and, apart from roundoff errors, which should be negligible due to the numerical stability of the method, computes the measure with well controlled and specifiable-in-advance error. The basic idea of the method is to obtain a truncated transformed model having, with some arbitrarily small error, the same interval availability distribution as the original model and solve the truncated transformed model using a state-of-the-art algorithm. The method requires the selection of a regenerative state and its performance depends on that selection. Due to its "benign" behavior, for large enough models and long enough mission times the new method is guaranteed to be significantly faster than previously proposed methods. For a class of models, class $\mathrm{C}_{1}$, including typical failure/repair models of coherent fault-tolerant systems with exponential failure and repair time distributions and repair in every state with failed components, a natural selection for the regenerative state exists and theoretical results are available assessing the performance of the method for that natural selection in terms of "visible" model characteristics. Those results can be used to anticipate when the new method can be expected to be significantly faster than previous methods.

\section{Acknowledgements}

The author wants to thank Víctor Suñe for implementing Algorithm A of [6], for his help in the implementation of the method proposed in this paper, and for helpful discussions during the early stages of this work. This work was supported by the "Comisión Internacional de Ciencia y Tecnología” (CICYT) under the research grants TIC95-0707-C02-02 and TAP1999-0443-C05-05.

\section{Appendix A.}

Lemma A1. Let $X=\{X(t) ; t \geqslant 0\}$ be a CTMC with finite state space $\Omega=U \cup D$, where the states in $U$ are up states and the states in $D$ are down states, and let $X^{\prime}$ be a CTMC differing from $X$ only in that the transition rate of $X$ from some state $i$ to some state $j$ has been increased by $\lambda>0$. Let $\operatorname{IAVCD}(t, p)$ be the interval availability complementary distribution of $X$ and let $\operatorname{IAVCD}^{\prime}(t, p)$ be the interval availability complementary distribution of $X^{\prime}$. Then $\mid \operatorname{IAVCD}^{\prime}(t, p)-$ $\operatorname{IAVCD}(t, p) \mid \leqslant 2 \lambda t$.

Proof. The proof is done using the formulation (1) for $\operatorname{IAVCD}(t, p)$ on which Algorithm A of [6] is based. We will reuse the notation introduced in Section 2, where that method has been reviewed, with $\Lambda \geqslant \max _{i \in \Omega} \lambda_{i}+\lambda$ so that both $X$ and $X^{\prime}$ can be randomized with rate $\Lambda$. In addition, we will denote using a prime the quantities referred to the CTMC $X^{\prime}$, e.g. $\mathbf{P}^{\prime}$ will denote the transition probability matrix of the randomized DTMC of $X^{\prime}$ with randomization rate $\Lambda$. 
Using the recurrences for the vectors $\mathbf{Y}_{n, k}$ given in Section 2 , for $n>0$ and $0<k \leqslant n$, we have

$$
\begin{aligned}
\mathbf{Y}_{n, k}^{\prime U}-\mathbf{Y}_{n, k}^{U} & =\mathbf{P}_{U, \Omega}^{\prime} \mathbf{Y}_{n-1, k-1}^{\prime}-\mathbf{P}_{U, \Omega} \mathbf{Y}_{n-1, k-1} \\
& =\mathbf{P}_{U, \Omega}^{\prime}\left(\mathbf{Y}_{n-1, k-1}^{\prime}-\mathbf{Y}_{n-1, k-1}\right)+\left(\mathbf{P}_{U, \Omega}^{\prime}-\mathbf{P}_{U, \Omega}\right) \mathbf{Y}_{n-1, k-1}
\end{aligned}
$$

from which

$$
\left\|\mathbf{Y}_{n, k}^{\prime U}-\mathbf{Y}_{n, k}^{U}\right\|_{\infty} \leqslant\left\|\mathbf{P}_{U, \Omega}^{\prime}\right\|_{\infty}\left\|\mathbf{Y}_{n-1, k-1}^{\prime}-\mathbf{Y}_{n-1, k-1}\right\|_{\infty}+\left\|\mathbf{P}_{U, \Omega}^{\prime}-\mathbf{P}_{U, \Omega}\right\|_{\infty}\left\|\mathbf{Y}_{n-1, k-1}\right\|_{\infty},
$$

and using $\left\|\mathbf{P}_{U, \Omega}^{\prime}\right\|_{\infty}=1,\left\|\mathbf{P}_{U, \Omega}^{\prime}-\mathbf{P}_{U, \Omega}\right\|_{\infty} \leqslant 2 \lambda / \Lambda$ and $\left\|\mathbf{Y}_{n-1, k-1}\right\|_{\infty} \leqslant 1$ :

$$
\left\|\mathbf{Y}_{n, k}^{\prime U}-\mathbf{Y}_{n, k}^{U}\right\|_{\infty} \leqslant\left\|\mathbf{Y}_{n-1, k-1}^{\prime}-\mathbf{Y}_{n-1, k-1}\right\|_{\infty}+\frac{2 \lambda}{\Lambda}, n>0,0<k \leqslant n .
$$

For $n>0, \mathbf{Y}_{n, 0}^{\prime U}=\mathbf{Y}_{n, 0}^{U}=\mathbf{1}$, and

$$
\left\|\mathbf{Y}_{n, 0}^{\prime U}-\mathbf{Y}_{n, 0}^{U}\right\|_{\infty}=0, n>0
$$

For $n>0$ and $0 \leqslant k<n$,

$$
\begin{aligned}
\mathbf{Y}_{n, k}^{\prime D}-\mathbf{Y}_{n, k}^{D} & =\mathbf{P}_{D, \Omega}^{\prime} \mathbf{Y}_{n-1, k}^{\prime}-\mathbf{P}_{D, \Omega} \mathbf{Y}_{n-1, k} \\
& =\mathbf{P}_{D, \Omega}^{\prime}\left(\mathbf{Y}_{n-1, k}^{\prime}-\mathbf{Y}_{n-1, k}\right)+\left(\mathbf{P}_{D, \Omega}^{\prime}-\mathbf{P}_{D, \Omega}\right) \mathbf{Y}_{n-1, k}
\end{aligned}
$$

from which

$$
\left\|\mathbf{Y}_{n, k}^{\prime D}-\mathbf{Y}_{n, k}^{D}\right\|_{\infty} \leqslant\left\|\mathbf{P}_{D, \Omega}^{\prime}\right\|_{\infty}\left\|\mathbf{Y}_{n-1, k}^{\prime}-\mathbf{Y}_{n-1, k}\right\|_{\infty}+\left\|\mathbf{P}_{D, \Omega}^{\prime}-\mathbf{P}_{D, \Omega}\right\|_{\infty}\left\|\mathbf{Y}_{n-1, k}\right\|_{\infty}
$$

and, using $\left\|\mathbf{P}_{D, \Omega}^{\prime}\right\|_{\infty}=1,\left\|\mathbf{P}_{D, \Omega}^{\prime}-\mathbf{P}_{D, \Omega}\right\|_{\infty} \leqslant 2 \lambda / \Lambda$ and $\left\|\mathbf{Y}_{n-1, k}\right\|_{\infty} \leqslant 1$,

$$
\left\|\mathbf{Y}_{n, k}^{\prime D}-\mathbf{Y}_{n, k}^{D}\right\|_{\infty} \leqslant\left\|\mathbf{Y}_{n-1, k}^{\prime}-\mathbf{Y}_{n-1, k}\right\|_{\infty}+\frac{2 \lambda}{\Lambda}, \quad n>0,0 \leqslant k<n .
$$

Finally, for $n>0, \mathbf{Y}_{n, n}^{\prime D}=\mathbf{Y}_{n, n}^{D}=\mathbf{0}$, and

$$
\left\|\mathbf{Y}_{n, n}^{\prime D}-\mathbf{Y}_{n, n}^{D}\right\|_{\infty}=0, n>0
$$

Summarizing (A.1)-(A.4) and using $\left\|\mathbf{Y}_{n, k}^{\prime}-\mathbf{Y}_{n, k}\right\|_{\infty}=\max \left\{\left\|\mathbf{Y}_{n, k}^{\prime U}-\mathbf{Y}_{n, k}^{U}\right\|_{\infty},\left\|\mathbf{Y}_{n, k}^{\prime D}-\mathbf{Y}_{n, k}^{D}\right\|_{\infty}\right\}$, we can write

$$
\max _{0 \leqslant k \leqslant n}\left\|\mathbf{Y}_{n, k}^{\prime}-\mathbf{Y}_{n, k}\right\|_{\infty} \leqslant \max _{0 \leqslant k \leqslant n-1}\left\|\mathbf{Y}_{n-1, k}^{\prime}-\mathbf{Y}_{n-1, k}\right\|_{\infty}+\frac{2 \lambda}{\Lambda}
$$

which with $\left\|\mathbf{Y}_{0,0}^{\prime}-\mathbf{Y}_{0,0}\right\|_{\infty}=0$ (because $\mathbf{Y}_{0,0}^{\prime}=\mathbf{Y}_{0,0}^{U}=\mathbf{1}$ and $\mathbf{Y}_{0,0}^{\prime D}=\mathbf{Y}_{0,0}^{D}=\mathbf{0}$ ) gives

$$
\max _{0 \leqslant k \leqslant n}\left\|\mathbf{Y}_{n, k}^{\prime}-\mathbf{Y}_{n, k}\right\|_{\infty} \leqslant n \frac{2 \lambda}{\Lambda}, n \geqslant 0
$$

and

$$
\left\|\mathbf{Y}_{n, k}^{\prime}-\mathbf{Y}_{n, k}\right\|_{\infty} \leqslant n \frac{2 \lambda}{\Lambda}, \quad n \geqslant 0, \quad 0 \leqslant k \leqslant n
$$


But

$$
Y_{n, k}^{\prime}-Y_{n, k}=\alpha \mathbf{Y}_{n, k}^{\prime}-\alpha \mathbf{Y}_{n, k}=\alpha\left(\mathbf{Y}_{n, k}^{\prime}-\mathbf{Y}_{n, k}\right)
$$

which, using $\|\boldsymbol{\alpha}\|_{\infty}=1$, implies

$$
\left|Y_{n, k}^{\prime}-Y_{n, k}\right| \leqslant\left\|\mathbf{Y}_{n, k}^{\prime}-\mathbf{Y}_{n, k}\right\|_{\infty} \leqslant n \frac{2 \lambda}{\Lambda}, n \geqslant 0,0 \leqslant k \leqslant n
$$

and

$$
\begin{aligned}
\left|\operatorname{IAVCD}^{\prime}(t, p)-\operatorname{IAVCD}(t, p)\right| & \leqslant \sum_{n=0}^{\infty} \mathrm{e}^{-\Lambda t} \frac{(\Lambda t)^{n}}{n !} \sum_{k=0}^{n}\left(\begin{array}{l}
n \\
k
\end{array}\right) p^{k} q^{n-k}\left|Y_{n, k}^{\prime}-Y_{n, k}\right| \\
& \leqslant \sum_{n=0}^{\infty} \mathrm{e}^{-\Lambda t} \frac{(\Lambda t)^{n}}{n !} \sum_{k=0}^{n}\left(\begin{array}{l}
n \\
k
\end{array}\right) p^{k} q^{n-k} n \frac{2 \lambda}{\Lambda}=\frac{2 \lambda}{\Lambda} \sum_{n=0}^{\infty} n \mathrm{e}^{-\Lambda t} \frac{(\Lambda t)^{n}}{n !} \\
& =\frac{2 \lambda}{\Lambda} \sum_{n=1}^{\infty} \mathrm{e}^{-\Lambda t} \frac{(\Lambda t)^{n}}{(n-1) !}=\frac{2 \lambda}{\Lambda} \Lambda t \sum_{n=0}^{\infty} \mathrm{e}^{-\Lambda t} \frac{(\Lambda t)^{n}}{n !}=2 \lambda t .
\end{aligned}
$$

Proof of Proposition 1. We will take into account that: (1) $\tilde{V}_{n} \in\left\{s_{l, k}^{u}, 0 \leqslant l \leqslant n, 1 \leqslant k \leqslant n+1\right\} \cup$ $\left\{s_{l, k}^{d}, 0 \leqslant l \leqslant n, 0 \leqslant k \leqslant n\right\} \cup\left\{s_{n, k}^{\prime \prime}, 1 \leqslant k \leqslant n+1\right\} \cup\left\{s_{n, k}^{\prime d}, 0 \leqslant k \leqslant n\right\} \cup\{f\}$, (2) $\tilde{V}_{n}=f$ implies $\tilde{V}_{n+1}=f$, (3) both $\tilde{V}_{n}=s_{l, k}^{u}$ and $\tilde{V}_{n}=s_{l, k}^{d}$ imply $\tilde{V}_{n+1} \in\left\{s_{0, .}, s_{l+1, k+1}^{u}, s_{l+1, k}^{d}, f\right\}$, and (4) both $\tilde{V}_{n}=s_{n, k}^{\prime u}$ and $\tilde{V}_{n}=s_{n, k}^{\prime d}$ imply $\tilde{V}_{n+1} \in\left\{s_{0, .}^{\cdot}, s_{n+1, k+1}^{\prime u}, s_{n+1, k}^{\prime d}, f\right\}$. From (2), it is clear that the only well-defined, non-null probability $P\left[\tilde{V}_{n+1}=y \mid \tilde{V}_{n}=x\right]$ for $x=f$ is $P\left[\tilde{V}_{n+1}=f \mid \tilde{V}_{n}=f\right]=1$. We will consider next the remaining cases for $x$ : (a) $x=s_{l, k}^{u}, 0 \leqslant l \leqslant n, 1 \leqslant k \leqslant n+1$, (b) $x=s_{l, k}^{d}, 0 \leqslant l \leqslant n, 0 \leqslant k \leqslant n$, (c) $x=s_{n, k}^{\prime \prime}, 1 \leqslant k \leqslant n+1$, and (d) $x=s_{n, k}^{\prime d}, 0 \leqslant k \leqslant n$.

Case a $\left(P\left[\tilde{V}_{n}=s_{l, k}^{u}\right]>0,0 \leqslant l \leqslant n, 1 \leqslant k \leqslant n+1\right.$, which implies (21) $\left.P\left[\hat{X}_{n-l}=r\right]>0\right)$ : Using the definition of $\tilde{V}(21)$, remembering that $\hat{X}^{\prime}$ is a version of $\hat{X}$ with initial state $r$, using the definition of $Z$ (2), and that $Z_{n} \neq r$ for $n>0$ :

$$
\begin{aligned}
& P\left[\tilde{V}_{n+1}=s_{l+1, k+1}^{u} \mid \tilde{V}_{n}=s_{l, k}^{u}\right] \\
& =\frac{P\left[\tilde{V}_{n}=s_{l, k}^{u} \wedge \tilde{V}_{n+1}=s_{l+1, k+1}^{u}\right]}{P\left[\tilde{V}_{n}=s_{l, k}^{u}\right]} \\
& =\frac{P\left[\hat{X}_{n-l}=r \wedge \hat{X}_{n-l+1: n+1} \in S^{\prime} \wedge \#\left(\hat{X}_{n-l: n} \in U_{S}\right)=k \wedge \hat{X}_{n} \in U_{S} \wedge \hat{X}_{n+1} \in U_{S}\right]}{P\left[\hat{X}_{n-l}=r \wedge \hat{X}_{n-l+1: n} \in S^{\prime} \wedge \#\left(\hat{X}_{n-l: n} \in U_{S}\right)=k \wedge \hat{X}_{n} \in U_{S}\right]} \\
& =\frac{P\left[\hat{X}_{n-l+1: n+1} \in S^{\prime} \wedge \#\left(\hat{X}_{n-l: n} \in U_{S}\right)=k \wedge \hat{X}_{n} \in U_{S} \wedge \hat{X}_{n+1} \in U_{S} \mid \hat{X}_{n-l}=r\right]}{P\left[\hat{X}_{n-l+1: n} \in S^{\prime} \wedge \#\left(\hat{X}_{n-l: n} \in U_{S}\right)=k \wedge \hat{X}_{n} \in U_{S} \mid \hat{X}_{n-l}=r\right]}
\end{aligned}
$$




$$
\begin{aligned}
& =\frac{P\left[\hat{X}_{1: l+1}^{\prime} \in S^{\prime} \wedge \#\left(\hat{X}_{0: l}^{\prime} \in U_{S}\right)=k \wedge \hat{X}_{l}^{\prime} \in U_{S} \wedge \hat{X}_{l+1}^{\prime} \in U_{S}\right]}{P\left[\hat{X}_{1: l}^{\prime} \in S^{\prime} \wedge \#\left(\hat{X}_{0: l}^{\prime} \in U_{S}\right)=k \wedge \hat{X}_{l}^{\prime} \in U_{S}\right]} \\
& =\frac{P\left[\#\left(Z_{0: l} \in U_{S}\right)=k \wedge Z_{l} \in U_{S} \wedge Z_{l+1} \in U_{S}^{\prime}\right]}{P\left[\#\left(Z_{0: l} \in U_{S}\right)=k \wedge Z_{l} \in U_{S}\right]}=\frac{\sum_{i \in U_{S}} \pi_{i}(l, k) P_{i, U_{S}^{\prime}}}{\sum_{i \in U_{S}} \pi_{i}(l, k)}=w_{l, k}^{u u},
\end{aligned}
$$

$$
\begin{aligned}
P\left[\tilde{V}_{n+1}=s_{l+1, k}^{d} \mid \tilde{V}_{n}=s_{l, k}^{u}\right] & \\
= & \frac{P\left[\tilde{V}_{n}=s_{l, k}^{u} \wedge \tilde{V}_{n+1}=s_{l+1, k}^{d}\right]}{P\left[\tilde{V}_{n}=s_{l, k}^{u}\right]} \\
= & \frac{P\left[\hat{X}_{n-l}=r \wedge \hat{X}_{n-l+1: n+1} \in S^{\prime} \wedge \#\left(\hat{X}_{n-l: n} \in U_{S}\right)=k \wedge \hat{X}_{n} \in U_{S} \wedge \hat{X}_{n+1} \in D_{S}\right]}{P\left[\hat{X}_{n-l}=r \wedge \hat{X}_{n-l+1: n} \in S^{\prime} \wedge \#\left(\hat{X}_{n-l: n} \in U_{S}\right)=k \wedge \hat{X}_{n} \in U_{S}\right]} \\
= & \frac{P\left[\hat{X}_{n-l+1: n+1} \in S^{\prime} \wedge \#\left(\hat{X}_{n-l: n} \in U_{S}\right)=k \wedge \hat{X}_{n} \in U_{S} \wedge \hat{X}_{n+1} \in D_{S} \mid \hat{X}_{n-l}=r\right]}{P\left[\hat{X}_{n-l+1: n} \in S^{\prime} \wedge \#\left(\hat{X}_{n-l: n} \in U_{S}\right)=k \wedge \hat{X}_{n} \in U_{S} \mid \hat{X}_{n-l}=r\right]} \\
= & \frac{P\left[\hat{X}_{1: l+1}^{\prime} \in S^{\prime} \wedge \#\left(\hat{X}_{0: l}^{\prime} \in U_{S}\right)=k \wedge \hat{X}_{l}^{\prime} \in U_{S} \wedge \hat{X}_{l+1}^{\prime} \in D_{S}\right]}{P\left[\hat{X}_{1: l}^{\prime} \in S^{\prime} \wedge \#\left(\hat{X}_{0: l}^{\prime} \in U_{S}\right)=k \wedge \hat{X}_{l}^{\prime} \in U_{S}\right]} \\
= & \frac{P\left[\#\left(Z_{0: l} \in U_{S}\right)=k \wedge Z_{l} \in U_{S} \wedge Z_{l+1} \in D_{S}^{\prime}\right]}{P\left[\#\left(Z_{0: l} \in U_{S}\right)=k \wedge Z_{l} \in U_{S}\right]}=\frac{\sum_{i \in U_{S}} \pi_{i}(l, k) P_{i, D_{S}^{\prime}}}{\sum_{i \in U_{S}} \pi_{i}(l, k)}=w_{l, k}^{u d},
\end{aligned}
$$

$$
\begin{aligned}
P\left[\tilde{V}_{n+1}=\dot{s_{0, \cdot}} \mid \tilde{V}_{n}=s_{l, k}^{u}\right] & \\
= & \frac{P\left[\tilde{V}_{n}=s_{l, k}^{u} \wedge \tilde{V}_{n+1}=s_{0, .}^{\cdot}\right]}{P\left[\tilde{V}_{n}=s_{l, k}^{u}\right]} \\
= & \frac{P\left[\hat{X}_{n-l}=r \wedge \hat{X}_{n-l+1: n} \in S^{\prime} \wedge \#\left(\hat{X}_{n-l: n} \in U_{S}\right)=k \wedge \hat{X}_{n} \in U_{S} \wedge \hat{X}_{n+1}=r\right]}{P\left[\hat{X}_{n-l}=r \wedge \hat{X}_{n-l+1: n} \in S^{\prime} \wedge \#\left(\hat{X}_{n-l: n} \in U_{S}\right)=k \wedge \hat{X}_{n} \in U_{S}\right]} \\
= & \frac{P\left[\hat{X}_{n-l+1: n} \in S^{\prime} \wedge \#\left(\hat{X}_{n-l: n} \in U_{S}\right)=k \wedge \hat{X}_{n} \in U_{S} \wedge \hat{X}_{n+1}=r \mid \hat{X}_{n-l}=r\right]}{P\left[\hat{X}_{n-l+1: n} \in S^{\prime} \wedge \#\left(\hat{X}_{n-l: n} \in U_{S}\right)=k \wedge \hat{X}_{n} \in U_{S} \mid \hat{X}_{n-l}=r\right]} \\
= & \frac{P\left[\hat{X}_{1: l}^{\prime} \in S^{\prime} \wedge \#\left(\hat{X}_{0: l}^{\prime} \in U_{S}\right)=k \wedge \hat{X}_{l}^{\prime} \in U_{S} \wedge \hat{X}_{l+1}^{\prime}=r\right]}{P\left[\hat{X}_{1: l}^{\prime} \in S^{\prime} \wedge \#\left(\hat{X}_{0: l}^{\prime} \in U_{S}\right)=k \wedge \hat{X}_{l}^{\prime} \in U_{S}\right]} \\
= & \frac{P\left[\#\left(Z_{0: l} \in U_{S}\right)=k \wedge Z_{l} \in U_{S} \wedge Z_{l+1}=a\right]}{P\left[\#\left(Z_{0: l} \in U_{S}\right)=k \wedge Z_{l} \in U_{S}\right]}=\frac{\sum_{i \in U_{S}} \pi_{i}(l, k) P_{i, r}}{\sum_{i \in U_{S}} \pi_{i}(l, k)}=q_{l, k}^{u},
\end{aligned}
$$




$$
\begin{aligned}
& P\left[\tilde{V}_{n+1}=f \mid \tilde{V}_{n}=s_{l, k}^{u}\right]=\frac{P\left[\tilde{V}_{n}=s_{l, k}^{u} \wedge \tilde{V}_{n+1}=f\right]}{P\left[\tilde{V}_{n}=s_{l, k}^{u}\right]} \\
& =\frac{P\left[\hat{X}_{n-l}=r \wedge \hat{X}_{n-l+1: n} \in S^{\prime} \wedge \#\left(\hat{X}_{n-l: n} \in U_{S}\right)=k \wedge \hat{X}_{n} \in U_{S} \wedge \hat{X}_{n+1}=f\right]}{P\left[\hat{X}_{n-l}=r \wedge \hat{X}_{n-l+1: n} \in S^{\prime} \wedge \#\left(\hat{X}_{n-l: n} \in U_{S}\right)=k \wedge \hat{X}_{n} \in U_{S}\right]} \\
& =\frac{P\left[\hat{X}_{n-l+1: n} \in S^{\prime} \wedge \#\left(\hat{X}_{n-l: n} \in U_{S}\right)=k \wedge \hat{X}_{n} \in U_{S} \wedge \hat{X}_{n+1}=f \mid \hat{X}_{n-l}=r\right]}{P\left[\hat{X}_{n-l+1: n} \in S^{\prime} \wedge \#\left(\hat{X}_{n-l: n} \in U_{S}\right)=k \wedge \hat{X}_{n} \in U_{S} \mid \hat{X}_{n-l}=r\right]} \\
& =\frac{P\left[\hat{X}_{1: l}^{\prime} \in S^{\prime} \wedge \#\left(\hat{X}_{0: l}^{\prime} \in U_{S}\right)=k \wedge \hat{X}_{l}^{\prime} \in U_{S} \wedge \hat{X}_{l+1}^{\prime}=f\right]}{P\left[\hat{X}_{1: l}^{\prime} \in S^{\prime} \wedge \#\left(\hat{X}_{0: l}^{\prime} \in U_{S}\right)=k \wedge \hat{X}_{l}^{\prime} \in U_{S}\right]} \\
& =\frac{P\left[\#\left(Z_{0: l} \in U_{S}\right)=k \wedge Z_{l} \in U_{S} \wedge Z_{l+1}=f\right]}{P\left[\#\left(Z_{0: l} \in U_{S}\right)=k \wedge Z_{l} \in U_{S}\right]}=\frac{\sum_{i \in U_{S}} \pi_{i}(l, k) P_{i, f}}{\sum_{i \in U_{S}} \pi_{i}(l, k)}=v_{l, k}^{u} .
\end{aligned}
$$

Case $b\left(P\left[\tilde{V}_{n}=s_{l, k}^{d}\right]>0,0 \leqslant l \leqslant n, 0 \leqslant k \leqslant n\right)$ : The developments are very similar to those for case a. The results are:

$$
\begin{aligned}
& P\left[\tilde{V}_{n+1}=s_{l+1, k+1}^{u} \mid \tilde{V}_{n}=s_{l, k}^{d}\right]=\frac{\sum_{i \in D_{S}} \pi_{i}(l, k) P_{i, U_{S}^{\prime}}}{\sum_{i \in D_{S}} \pi_{i}(l, k)}=w_{l, k}^{d u}, \\
& P\left[\tilde{V}_{n+1}=s_{l+1, k}^{d} \mid \tilde{V}_{n}=s_{l, k}^{d}\right]=\frac{\sum_{i \in D_{S}} \pi_{i}(l, k) P_{i, D_{S}^{\prime}}}{\sum_{i \in D_{S}} \pi_{i}(l, k)}=w_{l, k}^{d d}, \\
& P\left[\tilde{V}_{n+1}=s_{0, \cdot}^{\dot{1}} \mid \tilde{V}_{n}=s_{l, k}^{d}\right]=\frac{\sum_{i \in D_{S}} \pi_{i}(l, k) P_{i, r}}{\sum_{i \in D_{S}} \pi_{i}(l, k)}=q_{l, k}^{d}, \\
& P\left[\tilde{V}_{n+1}=f \mid \tilde{V}_{n}=s_{l, k}^{d}\right]=\frac{\sum_{i \in D_{S}} \pi_{i}(l, k) P_{i, f}}{\sum_{i \in D_{S}} \pi_{i}(l, k)}=v_{l, k}^{d} .
\end{aligned}
$$

Case $c\left(P\left[\tilde{V}_{n}=s_{n, k}^{\prime u}\right]>0,1 \leqslant k \leqslant n+1\right)$ : Using the definition of $\tilde{V}(21)$ and the definition of $Z^{\prime}(3)$ :

$$
\begin{aligned}
& P\left[\tilde{V}_{n+1}=s_{n+1, k+1}^{\prime u} \mid \tilde{V}_{n}=s_{n, k}^{\prime u}\right]=\frac{P\left[\tilde{V}_{n}=s_{n, k}^{\prime u} \wedge \tilde{V}_{n+1}=s_{n+1, k+1}^{\prime u}\right]}{P\left[\tilde{V}_{n}=s_{n, k}^{\prime u}\right]} \\
& =\frac{P\left[\hat{X}_{0: n+1} \in S^{\prime} \wedge \#\left(\hat{X}_{0: n} \in U_{S}^{\prime}\right)=k \wedge \hat{X}_{n} \in U_{S}^{\prime} \wedge \hat{X}_{n+1} \in U_{S}^{\prime}\right]}{P\left[\hat{X}_{0: n} \in S^{\prime} \wedge \#\left(\hat{X}_{0: n} \in U_{S}^{\prime}\right)=k \wedge \hat{X}_{n} \in U_{S}^{\prime}\right]} \\
& =\frac{P\left[\#\left(Z_{0: n}^{\prime} \in U_{S}^{\prime}\right)=k \wedge Z_{n}^{\prime} \in U_{S}^{\prime} \wedge Z_{n+1}^{\prime} \in U_{S}^{\prime}\right]}{P\left[\#\left(Z_{0: n}^{\prime} \in U_{S}^{\prime}\right)=k \wedge Z_{n}^{\prime} \in U_{S}^{\prime}\right]}=\frac{\sum_{i \in U_{S}^{\prime}} \pi_{i}^{\prime}(n, k) P_{i, U_{S}^{\prime}}}{\sum_{i \in U_{S}^{\prime}} \pi_{i}^{\prime}(n, k)}=w_{n, k}^{\prime \prime u},
\end{aligned}
$$




$$
\begin{aligned}
& P\left[\tilde{V}_{n+1}=s_{n+1, k}^{\prime d} \mid \tilde{V}_{n}=s_{n, k}^{\prime \prime}\right]=\frac{P\left[\tilde{V}_{n}=s_{n, k}^{\prime u} \wedge \tilde{V}_{n+1}=s_{n+1, k}^{\prime d}\right]}{P\left[\tilde{V}_{n}=s_{n, k}^{\prime u}\right]} \\
& =\frac{P\left[\hat{X}_{0: n+1} \in S^{\prime} \wedge \#\left(\hat{X}_{0: n} \in U_{S}^{\prime}\right)=k \wedge \hat{X}_{n} \in U_{S}^{\prime} \wedge \hat{X}_{n+1} \in D_{S}^{\prime}\right]}{P\left[\hat{X}_{0: n} \in S^{\prime} \wedge \#\left(\hat{X}_{0: n} \in U_{S}^{\prime}\right)=k \wedge \hat{X}_{n} \in U_{S}^{\prime}\right]} \\
& =\frac{P\left[\#\left(Z_{0: n}^{\prime} \in U_{S}^{\prime}\right)=k \wedge Z_{n}^{\prime} \in U_{S}^{\prime} \wedge Z_{n+1}^{\prime} \in D_{S}^{\prime}\right]}{P\left[\#\left(Z_{0: n}^{\prime} \in U_{S}^{\prime}\right)=k \wedge Z_{n}^{\prime} \in U_{S}^{\prime}\right]}=\frac{\sum_{i \in U_{S}^{\prime}} \pi_{i}^{\prime}(n, k) P_{i, D_{S}^{\prime}}}{\sum_{i \in U_{S}^{\prime}} \pi_{i}^{\prime}(n, k)}=w_{n, k}^{\prime u d}, \\
& P\left[\tilde{V}_{n+1}=s_{0, .} \mid \tilde{V}_{n}=s_{n, k}^{\prime u}\right]=\frac{P\left[\tilde{V}_{n}=s_{n, k}^{\prime u} \wedge \tilde{V}_{n+1}=s_{0, .}^{\cdot}\right]}{P\left[\tilde{V}_{n}=s_{n, k}^{\prime u}\right]} \\
& =\frac{P\left[\hat{X}_{0: n} \in S^{\prime} \wedge \#\left(\hat{X}_{0: n} \in U_{S}^{\prime}\right)=k \wedge \hat{X}_{n} \in U_{S}^{\prime} \wedge \hat{X}_{n+1}=r\right]}{P\left[\hat{X}_{0: n} \in S^{\prime} \wedge \#\left(\hat{X}_{0: n} \in U_{S}^{\prime}\right)=k \wedge \hat{X}_{n} \in U_{S}^{\prime}\right]} \\
& =\frac{P\left[\#\left(Z_{0: n}^{\prime} \in U_{S}^{\prime}\right)=k \wedge Z_{n}^{\prime} \in U_{S}^{\prime} \wedge Z_{n+1}^{\prime}=a\right]}{P\left[\#\left(Z_{0: n}^{\prime} \in U_{S}^{\prime}\right)=k \wedge Z_{n}^{\prime} \in U_{S}^{\prime}\right]}=\frac{\sum_{i \in U_{S}^{\prime}} \pi_{i}^{\prime}(n, k) P_{i, r}}{\sum_{i \in U_{S}^{\prime}} \pi_{i}^{\prime}(n, k)}=q_{n, k}^{\prime u}, \\
& P\left[\tilde{V}_{n+1}=f \mid \tilde{V}_{n}=s_{n, k}^{\prime u}\right]=\frac{P\left[\tilde{V}_{n}=s_{n, k}^{\prime u} \wedge \tilde{V}_{n+1}=f\right]}{P\left[\tilde{V}_{n}=s_{n, k}^{\prime u}\right]} \\
& =\frac{P\left[\hat{X}_{0: n} \in S^{\prime} \wedge \#\left(\hat{X}_{0: n} \in U_{S}^{\prime}\right)=k \wedge \hat{X}_{n} \in U_{S}^{\prime} \wedge \hat{X}_{n+1}=f\right]}{P\left[\hat{X}_{0: n} \in S^{\prime} \wedge \#\left(\hat{X}_{0: n} \in U_{S}^{\prime}\right)=k \wedge \hat{X}_{n} \in U_{S}^{\prime}\right]} \\
& =\frac{P\left[\#\left(Z_{0: n}^{\prime} \in U_{S}^{\prime}\right)=k \wedge Z_{n}^{\prime} \in U_{S}^{\prime} \wedge Z_{n+1}^{\prime}=f\right]}{P\left[\#\left(Z_{0: n}^{\prime} \in U_{S}^{\prime}\right)=k \wedge Z_{n}^{\prime} \in U_{S}^{\prime}\right]}=\frac{\sum_{i \in U_{S}^{\prime}} \pi_{i}^{\prime}(n, k) P_{i, f}}{\sum_{i \in U_{S}^{\prime}} \pi_{i}^{\prime}(n, k)}=v_{n, k}^{\prime u} .
\end{aligned}
$$

Case $d\left(P\left[\tilde{V}_{n}=s_{n, k}^{\prime d}\right]>0,0 \leqslant k \leqslant n\right)$ : The developments are very similar to those of case c. The results are:

$$
\begin{gathered}
P\left[\tilde{V}_{n+1}=s_{n+1, k+1}^{\prime u} \mid \tilde{V}_{n}=s_{n, k}^{\prime d}\right]=\frac{\sum_{i \in D_{S}^{\prime}} \pi_{i}^{\prime}(n, k) P_{i, U_{S}^{\prime}}}{\sum_{i \in D_{S}^{\prime}} \pi_{i}^{\prime}(n, k)}=w_{n, k}^{\prime d u}, \\
P\left[\tilde{V}_{n+1}=s_{n+1, k}^{\prime d} \mid \tilde{V}_{n}=s_{n, k}^{\prime d}\right]=\frac{\sum_{i \in D_{S}^{\prime}} \pi_{i}^{\prime}(n, k) P_{i, D_{S}^{\prime}}}{\sum_{i \in D_{S}^{\prime}} \pi_{i}^{\prime}(n, k)}=w_{n, k}^{\prime d d},
\end{gathered}
$$




$$
\begin{aligned}
& P\left[\tilde{V}_{n+1}=s_{0, .} \mid \tilde{V}_{n}=s_{n, k}^{\prime d}\right]=\frac{\sum_{i \in D_{S}^{\prime}} \pi_{i}^{\prime}(n, k) P_{i, r}}{\sum_{i \in D_{S}^{\prime}} \pi_{i}^{\prime}(n, k)}=q_{n, k}^{\prime d}, \\
& P\left[\tilde{V}_{n+1}=f \mid \tilde{V}_{n}=s_{n, k}^{\prime d}\right]=\frac{\sum_{i \in D_{S}^{\prime}} \pi_{i}^{\prime}(n, k) P_{i, f}}{\sum_{i \in D_{S}^{\prime}} \pi_{i}^{\prime}(n, k)}=v_{n, k}^{\prime d} .
\end{aligned}
$$

Proof of Proposition 2. Assuming $P\left[\tilde{V}_{m}=s_{0,}\right.$. $]>0$, let (note that the definitions are correct because the steps at which $\tilde{V}$ hits state $\dot{s}$, are regeneration points)

$$
\begin{aligned}
& \tilde{v}(n, k)=P\left[\tilde{V}_{m+n}=f \wedge \tilde{V}_{m+1: m+n-1} \in{ }^{V} S^{\prime} \wedge \#\left(\tilde{V}_{m: m+n-1} \in{ }^{V} U_{S}\right)=k \mid \tilde{V}_{m}=\dot{s_{0,} .}\right] \\
& \tilde{q}(n, k)=P\left[\tilde{V}_{m+n}=\dot{s_{0, \cdot}} \wedge \tilde{V}_{m+1: m+n-1} \in{ }^{V} S^{\prime} \wedge \#\left(\tilde{V}_{m: m+n-1} \in{ }^{V} U_{S}\right)=k \mid \tilde{V}_{m}=\dot{s_{0, .}}\right] \\
& \tilde{w}^{u}(n, k)=P\left[\tilde{V}_{m+1: m+n} \in{ }^{V} S^{\prime} \wedge \#\left(\tilde{V}_{m: m+n} \in{ }^{V} U_{S}\right)=k \wedge \tilde{V}_{m+n} \in{ }^{V} U_{S} \mid \tilde{V}_{m}=\dot{s_{0,} \cdot}\right] \\
& \tilde{w}^{d}(n, k)=P\left[\tilde{V}_{m+1: m+n} \in{ }^{V} S^{\prime} \wedge \#\left(\tilde{V}_{m: m+n} \in{ }^{V} U_{S}\right)=k \wedge \tilde{V}_{m+n} \in{ }^{V} D_{S} \mid \tilde{V}_{m}=\dot{s_{0,} \cdot}\right],
\end{aligned}
$$

and let

$$
\begin{aligned}
& \tilde{v}^{\prime}(n, k)=P\left[\tilde{V}_{n}=f \wedge \tilde{V}_{0: n-1} \in{ }^{V} S^{\prime} \wedge \#\left(\tilde{V}_{0: n-1} \in{ }^{V} U_{S}^{\prime}\right)=k\right] \\
& \tilde{q}^{\prime}(n, k)=P\left[\tilde{V}_{n}=\dot{s_{0, \cdot}} \wedge \tilde{V}_{0: n-1} \in{ }^{V} S^{\prime} \wedge \#\left(\tilde{V}_{0: n-1} \in{ }^{V} U_{S}^{\prime}\right)=k\right], \\
& \tilde{w}^{\prime u}(n, k)=P\left[\tilde{V}_{0: n} \in{ }^{V} S^{\prime} \wedge \#\left(\tilde{V}_{0: n} \in{ }^{V} U_{S}^{\prime}\right)=k \wedge \tilde{V}_{n} \in{ }^{V} U_{S}^{\prime}\right] \\
& \tilde{w}^{\prime d}(n, k)=P\left[\tilde{V}_{0: n} \in{ }^{V} S^{\prime} \wedge \#\left(\tilde{V}_{0: n} \in{ }^{V} U_{S}^{\prime}\right)=k \wedge \tilde{V}_{n} \in{ }^{V} D_{S}^{\prime}\right] .
\end{aligned}
$$

Assuming $P\left[\tilde{V}_{m}=s_{0,}.\right]>0$, let

$$
\begin{aligned}
\tilde{\varphi}_{i}(n, k)= & P\left[\tilde{V}_{m+n}=\dot{s_{0, \cdot}} \wedge \tilde{V}_{m+1: m+n-1} \in{ }^{V} S \wedge \#\left(\tilde{V}_{m: m+n-1} \in{ }^{V} U_{S}\right)=k\right. \\
& \left.\wedge \#\left(\tilde{V}_{m+1: m+n-1}=\dot{s_{0,}}\right)=i \mid \tilde{V}_{m}=\dot{s_{0,}}\right] .
\end{aligned}
$$

Using the fact that the steps at which $\tilde{V}$ hits state $s_{0 \text {, }}$ are regeneration points, the probabilities $\tilde{\varphi}_{i}(n, k), 0 \leqslant i \leqslant n-1$ can be computed recursively using:

$$
\begin{aligned}
& \tilde{\varphi}_{0}(n, k)=\tilde{q}(n, k), \\
& \tilde{\varphi}_{i}(n, k)=\sum_{m=i}^{n-1} \sum_{l=0}^{\min \{k, m\}} \tilde{\varphi}_{i-1}(m, l) \tilde{q}(n-m, k-l), \quad i \geqslant 1 .
\end{aligned}
$$


Let

$$
\begin{aligned}
& \tilde{\phi}_{i}^{u}(n, k)=P\left[\#\left(\tilde{V}_{0: n} \in{ }^{V} U\right)=k \wedge \#\left(\tilde{V}_{0: n}=s_{0, .}\right)=i \wedge \tilde{V}_{n} \in{ }^{V} U\right], \\
& \tilde{\phi}_{i}^{d}(n, k)=P\left[\#\left(\tilde{V}_{0: n} \in{ }^{V} U\right)=k \wedge \#\left(\tilde{V}_{0: n}=\dot{s_{0, .}}\right)=i \wedge \tilde{V}_{n} \in{ }^{V} D\right] .
\end{aligned}
$$

We clearly have

$$
\begin{aligned}
& P\left[\#\left(\tilde{V}_{0: n} \in{ }^{V} U\right)=k \wedge \tilde{V}_{n} \in{ }^{V} U\right]=\sum_{i=0}^{n+1} \tilde{\phi}_{i}^{u}(n, k), \\
& P\left[\#\left(\tilde{V}_{0: n} \in{ }^{V} U\right)=k \wedge \tilde{V}_{n} \in{ }^{V} D\right]=\sum_{i=0}^{n+1} \tilde{\phi}_{i}^{d}(n, k) .
\end{aligned}
$$

Furthermore, the probabilities $\tilde{\phi}_{i}^{u}(n, k)$ and $\tilde{\phi}_{i}^{d}(n, k), 0 \leqslant i \leqslant n+1$ can be computed from $\tilde{v}(n, k)$, $\tilde{q}(n, k), \tilde{w}^{u}(n, k), \tilde{w}^{d}(n, k), \tilde{v}^{\prime}(n, k), \tilde{q}^{\prime}(n, k), \tilde{w}^{\prime l}(n, k), \tilde{w}^{\prime d}(n, k)$, and $\tilde{\varphi}_{i}(n, k), i \geqslant 0$ using:

$$
\begin{aligned}
& \tilde{\phi}_{0}^{u}(n, k)= \begin{cases}\tilde{w}^{\prime \prime}(n, k)+\sum_{m=\max \{0, n+1-k\}}^{n} \tilde{v}^{\prime}(m, k+m-n-1) & \text { if } f \in U, \\
\tilde{w}^{\prime \prime}(n, k) & \text { if } f \in D,\end{cases} \\
& \tilde{\phi}_{0}^{d}(n, k)= \begin{cases}\tilde{w}^{\prime d}(n, k) & \text { if } f \in U, \\
\tilde{w}^{\prime d}(n, k)+\sum_{m=k}^{n} \tilde{v}^{\prime}(m, k) & \text { if } f \in D,\end{cases} \\
& \tilde{\phi}_{1}^{u}(n, k)=\left\{\begin{array}{l}
\sum_{m_{1}=1}^{n} \sum_{l=\max \left\{0, k+m_{1}-n-1\right\}}^{\min \left\{k, m_{1}\right\}} \tilde{q}^{\prime}\left(m_{1}, l\right)\left(\tilde{w}^{u}\left(n-m_{1}, k-l\right)\right. \\
\left.+\sum_{m_{2}=\max \left\{1, n+l+1-k-m_{1}\right\}}^{n-m_{1}} \tilde{v}\left(m_{2}, k+m_{1}+m_{2}-n-l-1\right)\right) \\
+\alpha_{r}\left(\tilde{w}^{u}(n, k)+\sum_{m_{1}=\max \{1, n+1-k\}}^{n} \tilde{v}\left(m_{1}, k+m_{1}-n-1\right)\right) \text { if } f \in U, \\
\sum_{m_{1}=1}^{n} \sum_{l=\max \left\{0, k+m_{1}-n-1\right\}}^{n} \tilde{q}^{\prime}\left(m_{1}, l\right) \tilde{w}^{u}\left(n-m_{1}, k-l\right)+\alpha_{r} \tilde{w}^{u}(n, k) \text { if } f \in D,
\end{array}\right.
\end{aligned}
$$




$$
\tilde{\phi}_{1}^{d}(n, k)=\left\{\begin{array}{c}
\sum_{m_{1}=1}^{n} \sum_{l=\max \left\{0, k+m_{1}-n\right\}}^{\min \left\{k, m_{1}\right\}} \tilde{q}^{\prime}\left(m_{1}, l\right) \tilde{w}^{d}\left(n-m_{1}, k-l\right)+\alpha_{r} \tilde{w}^{d}(n, k) \text { if } f \in U, \\
\sum_{m_{1}=1}^{n} \sum_{l=\max \left\{0, k+m_{1}-n\right\}}^{\min \left\{k, m_{1}\right\}} \tilde{q}^{\prime}\left(m_{1}, l\right)\left(\tilde{w}^{d}\left(n-m_{1}, k-l\right)\right. \\
\left.+\sum_{m_{2}=\max ^{n-m_{1}}\{1, k-l\}} \tilde{v}\left(m_{2}, k-l\right)\right) \\
+\alpha_{r}\left(\tilde{w}^{d}(n, k)+\sum_{m_{1}=\max \{1, k\}}^{n} \tilde{v}\left(m_{1}, k\right)\right) \text { if } f \in D
\end{array}\right.
$$

and, for $i \geqslant 2$,

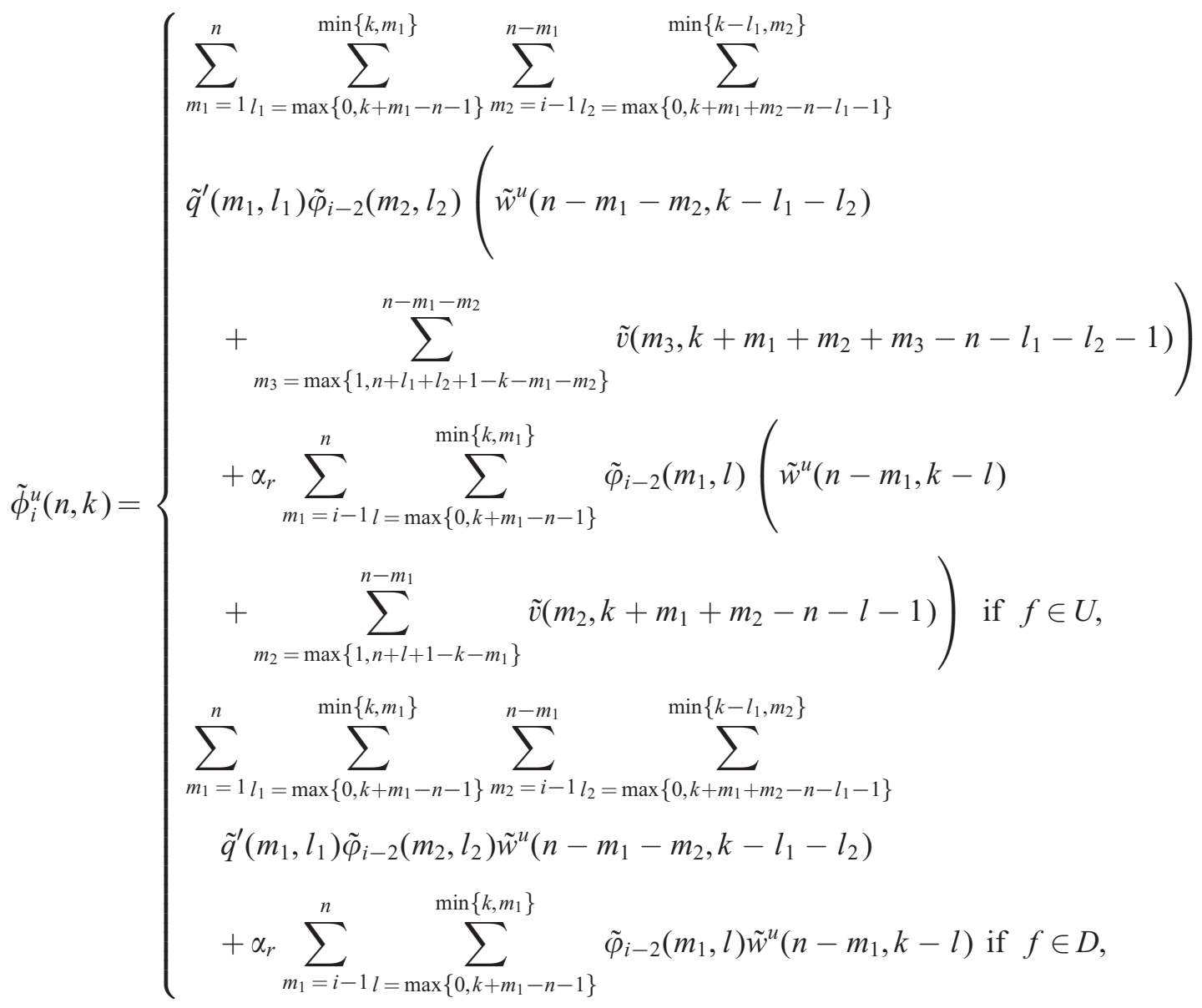




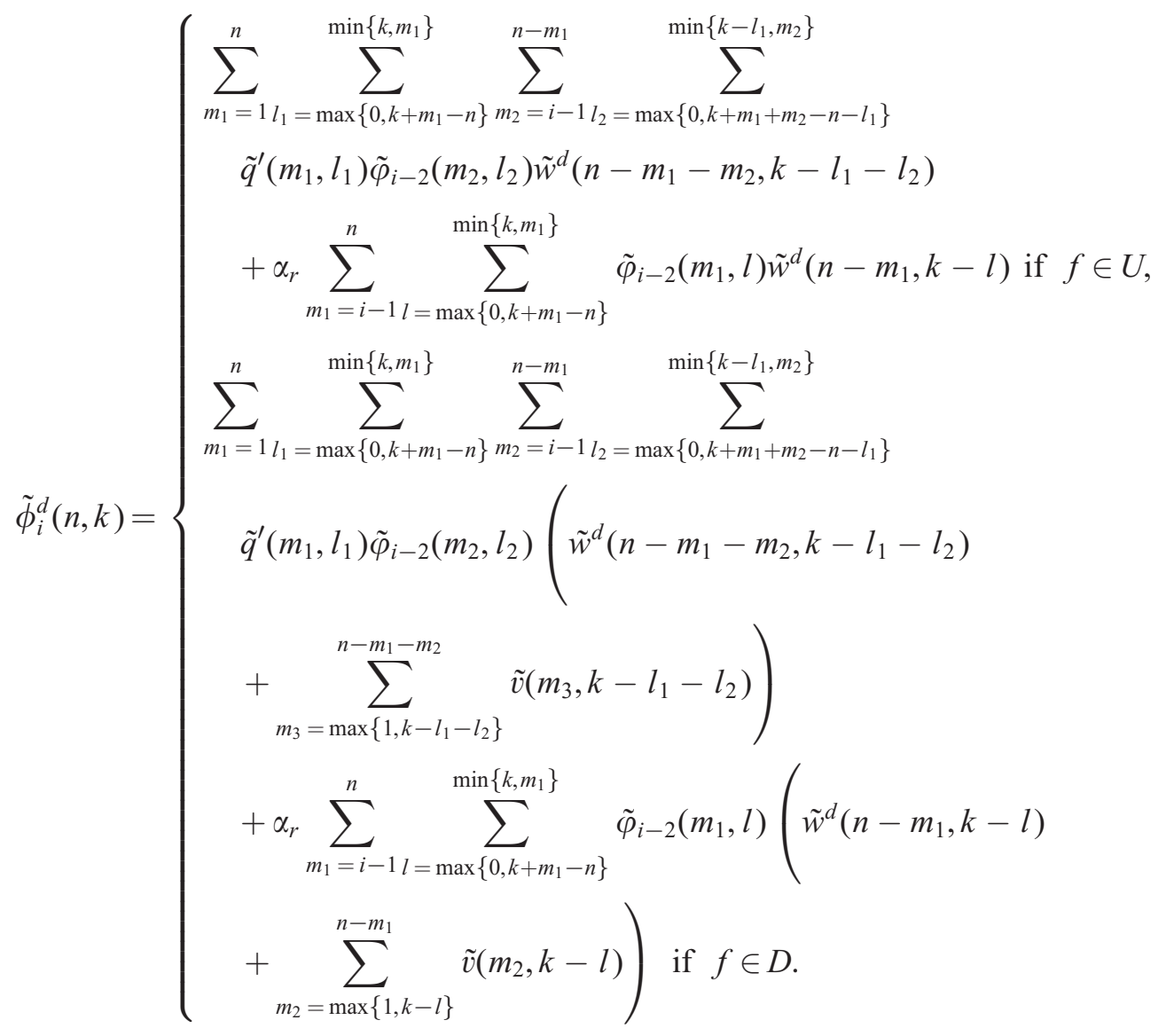

The developments made so far formalize $P\left[\#\left(\tilde{V}_{0: n} \in{ }^{V} U\right)=k \wedge \tilde{V}_{n} \in{ }^{V} U\right]$ and $P\left[\#\left(\tilde{V}_{0: n} \in{ }^{V} U\right)=k \wedge\right.$ $\left.\tilde{V}_{n} \in{ }^{V} D\right]$ in terms of $\tilde{v}(n, k), \tilde{q}(n, k), \tilde{w}^{u}(n, k), \tilde{w}^{d}(n, k), \tilde{v}^{\prime}(n, k), \tilde{q}^{\prime}(n, k), \tilde{w}^{\prime u}(n, k)$, and $\tilde{w}^{\prime d}(n, k)$.

Assuming $P\left[\hat{V}_{m}=s_{0,}^{\cdot}\right]>0$, let

$$
\begin{aligned}
& \hat{v}(n, k)=P\left[\hat{V}_{m+n}=f \wedge \hat{V}_{m+1: m+n-1} \in{ }^{V} S^{\prime} \wedge \#\left(\hat{V}_{m: m+n-1} \in{ }^{V} U_{S}\right)=k \mid \hat{V}_{m}=\dot{s_{0,}}\right] \\
& \hat{q}(n, k)=P\left[\hat{V}_{m+n}=\dot{s_{0, \cdot}} \wedge \hat{V}_{m+1: m+n-1} \in{ }^{V} S^{\prime} \wedge \#\left(\hat{V}_{m: m+n-1} \in{ }^{V} U_{S}\right)=k \mid \hat{V}_{m}=\dot{s_{0, .}}\right] \\
& \hat{w}^{u}(n, k)=P\left[\hat{V}_{m+1: m+n} \in{ }^{V} S^{\prime} \wedge \#\left(\hat{V}_{m: m+n} \in{ }^{V} U_{S}\right)=k \wedge \hat{V}_{m+n} \in{ }^{V} U_{S} \mid \hat{V}_{m}=\dot{s_{0,}} \cdot\right] \\
& \hat{w}^{d}(n, k)=P\left[\hat{V}_{m+1: m+n} \in{ }^{V} S^{\prime} \wedge \#\left(\hat{V}_{m: m+n} \in{ }^{V} U_{S}\right)=k \wedge \hat{V}_{m+n} \in{ }^{V} D_{S} \mid \hat{V}_{m}=\dot{s_{0,}}\right]
\end{aligned}
$$

and let

$$
\begin{aligned}
& \hat{v}^{\prime}(n, k)=P\left[\hat{V}_{n}=f \wedge \hat{V}_{0: n-1} \in{ }^{V} S^{\prime} \wedge \#\left(\hat{V}_{0: n-1} \in{ }^{V} U_{S}^{\prime}\right)=k\right], \\
& \hat{q}^{\prime}(n, k)=P\left[\hat{V}_{n}=\dot{s} \cdot \wedge \hat{V}_{0: n-1} \in{ }^{V} S^{\prime} \wedge \#\left(\hat{V}_{0: n-1} \in{ }^{V} U_{S}^{\prime}\right)=k\right],
\end{aligned}
$$




$$
\begin{aligned}
& \hat{w}^{\prime u}(n, k)=P\left[\hat{V}_{0: n} \in{ }^{V} S^{\prime} \wedge \#\left(\hat{V}_{0: n} \in{ }^{V} U_{S}^{\prime}\right)=k \wedge \hat{V}_{n} \in{ }^{V} U_{S}^{\prime}\right], \\
& \hat{w}^{\prime d}(n, k)=P\left[\hat{V}_{0: n} \in{ }^{V} S^{\prime} \wedge \#\left(\hat{V}_{0: n} \in{ }^{V} U_{S}^{\prime}\right)=k \wedge \hat{V}_{n} \in{ }^{V} D_{S}^{\prime}\right] .
\end{aligned}
$$

It is possible to formalize $P\left[\#\left(\hat{V}_{0: n} \in{ }^{V} U\right)=k \wedge \hat{V}_{n} \in{ }^{V} U\right]$ and $P\left[\#\left(\hat{V}_{0: n} \in{ }^{V} U\right)=k \wedge \hat{V}_{n} \in{ }^{V} D\right]$ in terms of $\hat{v}(n, k), \hat{q}(n, k), \hat{w}^{u}(n, k), \hat{w}^{d}(n, k), \hat{v}^{\prime}(n, k), \hat{q}^{\prime}(n, k), \hat{w}^{\prime u}(n, k)$, and $\hat{w}^{\prime d}(n, k)$ in a similar way. Therefore, to show that $P\left[\#\left(\hat{V}_{0: n} \in{ }^{V} U\right)=k \wedge \hat{V}_{n} \in{ }^{V} U\right]=P\left[\#\left(\tilde{V}_{0: n} \in{ }^{V} U\right)=k \wedge \tilde{V}_{n} \in{ }^{V} U\right]$ and $P\left[\#\left(\hat{V}_{0: n} \in{ }^{V} U\right)=k \wedge \hat{V}_{n} \in{ }^{V} D\right]=P\left[\#\left(\tilde{V}_{0: n} \in{ }^{V} U\right)=k \wedge \tilde{V}_{n} \in{ }^{V} D\right]$, is suffices to prove that: (1) $\hat{w}^{u}(n, k)=\tilde{w}^{u}(n, k), \quad \hat{w}^{d}(n, k)=\tilde{w}^{d}(n, k)$, (2) $\hat{v}(n, k)=\tilde{v}(n, k)$, (3) $\hat{q}(n, k)=\tilde{q}(n, k)$, (4) $\hat{w}^{\prime u}(n, k)=$ $\tilde{w}^{\prime \prime}(n, k), \hat{w}^{\prime d}(n, k)=\tilde{w}^{\prime d}(n, k),(5) \hat{v}^{\prime}(n, k)=\tilde{v}^{\prime}(n, k)$, and $(6) \hat{q}^{\prime}(n, k)=\tilde{q}^{\prime}(n, k)$. We will prove next all those results. In the proofs we will use (assuming $P\left[\hat{V}_{m}=s_{0,}^{\cdot}.\right]>0$ ):

$$
\begin{aligned}
& \hat{w}^{u}(n, k)=P\left[\hat{V}_{m+n}=s_{n, k}^{u} \mid \hat{V}_{m}=s_{0, .}\right], \\
& \hat{w}^{d}(n, k)=P\left[\hat{V}_{m+n}=s_{n, k}^{d} \mid \hat{V}_{m}=s_{0, .}\right] \\
& \hat{w}^{\prime u}(n, k)=P\left[\hat{V}_{n}=s_{n, k}^{\prime u}\right], \\
& \hat{w}^{\prime d}(n, k)=P\left[\hat{V}_{n}=s_{n, k}^{\prime d}\right],
\end{aligned}
$$

which easily follow from the definitions of $\hat{w}^{u}(n, k), \hat{w}^{d}(n, k), \hat{w}^{\prime u}(n, k)$, and $\hat{w}^{\prime d}(n, k)$ and the state transition diagram of $\hat{V}$ (illustrated in Fig. 2), taking into account that the up states of $\hat{V}$ in ${ }^{V} S$ (states in ${ }^{V} U_{S}$ ) are the states $s_{l, k}^{u}$ and $s_{l, k}^{\prime \prime}$ and the down states of $\hat{V}$ in ${ }^{V} S$ (states in ${ }^{V} D_{S}$ ) are the states $s_{l, k}^{d}$ and $s_{l, k}^{\prime d}$. Also, to avoid excessive discussion, we will conventionally assume that 0 by a non-defined quantity is 0 .

Proof of $(1)\left(\hat{w}^{u}(n, k)=\tilde{w}^{u}(n, k), \hat{w}^{d}(n, k)=\tilde{w}^{d}(n, k)\right)$. The proof is by induction on $n$. For the base case, $n=0$, we trivially have

$$
\begin{aligned}
& \hat{w}^{u}(0,0)=\tilde{w}^{u}(0,0)=0, \\
& \hat{w}^{u}(0,1)=\tilde{w}^{u}(0,1)= \begin{cases}1 & \text { if } r \in U_{S}, \\
0 & \text { if } r \in D_{S},\end{cases} \\
& \hat{w}^{d}(0,0)=\tilde{w}^{d}(0,0)= \begin{cases}0 & \text { if } r \in U_{S}, \\
1 & \text { if } r \in D_{S},\end{cases} \\
& \hat{w}^{d}(0,1)=\tilde{w}^{d}(0,1)=0 .
\end{aligned}
$$

Let us proceed with the induction step. First, using the definitions of $\tilde{w}^{u}(n, k)$ (A.6) and $\tilde{V}$ (21), remembering that $\hat{X}^{\prime}$ is a version of $\hat{X}$ with initial state $r$, and using the definition of $Z(2)$, assuming $P\left[\tilde{V}_{m}=s_{0,}^{\cdot}\right]>0$, which implies $P\left[\hat{X}_{m}=r\right]>0$ :

$$
\tilde{w}^{u}(n, k)=P\left[\hat{X}_{m+1: m+n} \in S^{\prime} \wedge \#\left(\hat{X}_{m: m+n} \in U_{S}\right)=k \wedge \hat{X}_{m+n} \in U_{S} \mid \hat{X}_{m}=r\right]
$$




$$
\begin{aligned}
& =P\left[\hat{X}_{1: n}^{\prime} \in S^{\prime} \wedge \#\left(\hat{X}_{0: n}^{\prime} \in U_{S}\right)=k \wedge \hat{X}_{n}^{\prime} \in U_{S}\right] \\
& =P\left[\#\left(Z_{0: n} \in U_{S}\right)=k \wedge Z_{n} \in U_{S}\right]=\sum_{i \in U_{S}} \pi_{i}(n, k), .
\end{aligned}
$$

Similarly, assuming $P\left[\tilde{V}_{m}=s_{0,}^{\cdot}\right]>0$, which implies $P\left[\hat{X}_{m}=r\right]>0$ :

$$
\begin{aligned}
& \tilde{w}^{d}(n, k)=P\left[\hat{X}_{m+1: m+n} \in S^{\prime} \wedge \#\left(\hat{X}_{m: m+n} \in U_{S}\right)=k \wedge \hat{X}_{m+n} \in D_{S} \mid \hat{X}_{m}=r\right] \\
& \quad=P\left[\hat{X}_{1: n}^{\prime} \in S^{\prime} \wedge \#\left(\hat{X}_{0: n}^{\prime} \in U_{S}\right)=k \wedge \hat{X}_{n}^{\prime} \in D_{S}\right] \\
& \quad=P\left[\#\left(Z_{0: n} \in U_{S}\right)=k \wedge Z_{n} \in D_{S}\right]=\sum_{i \in D_{S}} \pi_{i}(n, k) .
\end{aligned}
$$

On the other hand, considering the state transition diagram of $\hat{V}$ and using the induction hypothesis, (A.11), (A.12), and (4)-(6), assuming $P\left[\hat{V}_{m}=s_{0,}.\right]>0$ :

$$
\begin{aligned}
& \hat{w}^{u}(n+1, k)=P\left[\hat{V}_{m+n+1}=s_{n+1, k}^{u} \mid \hat{V}_{m}=\dot{s} \cdot\right] \\
& =P\left[\hat{V}_{m+n+1}=s_{n+1, k}^{u} \mid \hat{V}_{m+n}=s_{n, k-1}^{u}\right] P\left[\hat{V}_{m+n}=s_{n, k-1}^{u} \mid \hat{V}_{m}=s_{0, .}\right] \\
& +P\left[\hat{V}_{m+n+1}=s_{n+1, k}^{u} \mid \hat{V}_{m+n}=s_{n, k-1}^{d}\right] P\left[\hat{V}_{m+n}=s_{n, k-1}^{d} \mid \hat{V}_{m}=\dot{s} \cdot\right] \\
& =w_{n, k-1}^{u u} \hat{w}^{u}(n, k-1)+w_{n, k-1}^{d u} \hat{w}^{d}(n, k-1) \\
& =\frac{\sum_{i \in U_{S}} \pi_{i}(n, k-1) P_{i, U_{S}^{\prime}}}{\sum_{i \in U_{S}} \pi_{i}(n, k-1)} \tilde{w}^{u}(n, k-1)+\frac{\sum_{i \in D_{S}} \pi_{i}(n, k-1) P_{i, U_{S}^{\prime}}}{\sum_{i \in D_{S}} \pi_{i}(n, k-1)} \tilde{w}^{d}(n, k-1) \\
& =\sum_{i \in U_{S}} \pi_{i}(n, k-1) P_{i, U_{S}^{\prime}}+\sum_{i \in D_{S}} \pi_{i}(n, k-1) P_{i, U_{S}^{\prime}}=\sum_{i \in S} \pi_{i}(n, k-1) P_{i, U_{S}^{\prime}} \\
& =\sum_{i \in U_{S}^{\prime}} \pi_{i}(n+1, k)=\sum_{i \in U_{S}} \pi_{i}(n+1, k)=\tilde{w}^{u}(n+1, k), \\
& \hat{w}^{d}(n+1, k)=P\left[\hat{V}_{m+n+1}=s_{n+1, k}^{d} \mid \hat{V}_{m}=\dot{s} \cdot\right] \\
& =P\left[\hat{V}_{m+n+1}=s_{n+1, k}^{d} \mid \hat{V}_{m+n}=s_{n, k}^{u}\right] P\left[\hat{V}_{m+n}=s_{n, k}^{u} \mid \hat{V}_{m}=s_{0, .}\right] \\
& +P\left[\hat{V}_{m+n+1}=s_{n+1, k}^{d} \mid \hat{V}_{m+n}=s_{n, k}^{d}\right] P\left[\hat{V}_{m+n}=s_{n, k}^{d} \mid \hat{V}_{m}=\dot{s_{0, .}}\right] \\
& =w_{n, k}^{u d} \hat{w}^{u}(n, k)+w_{n, k}^{d d} \hat{w}^{d}(n, k) \\
& =\frac{\sum_{i \in U_{S}} \pi_{i}(n, k) P_{i, D_{S}^{\prime}}}{\sum_{i \in U_{S}} \pi_{i}(n, k)} \tilde{w}^{u}(n, k)+\frac{\sum_{i \in D_{S}} \pi_{i}(n, k) P_{i, D_{S}^{\prime}}}{\sum_{i \in D_{S}} \pi_{i}(n, k)} \tilde{w}^{d}(n, k)
\end{aligned}
$$




$$
\begin{aligned}
& =\sum_{i \in U_{S}} \pi_{i}(n, k) P_{i, D_{S}^{\prime}}+\sum_{i \in D_{S}} \pi_{i}(n, k) P_{i, D_{S}^{\prime}}=\sum_{i \in S} \pi_{i}(n, k) P_{i, D_{S}^{\prime}} \\
& =\sum_{i \in D_{S}^{\prime}} \pi_{i}(n+1, k)=\sum_{i \in D_{S}} \pi_{i}(n+1, k)=\tilde{w}^{d}(n+1, k) .
\end{aligned}
$$

Proof of (2) $(\hat{v}(n, k)=\tilde{v}(n, k))$. Using the definitions of $\tilde{v}(n, k)(\mathrm{A} .5)$ and $\tilde{V}(21)$, remembering that $\hat{X}^{\prime}$ is a version of $\hat{X}$ with initial state $r$ and using the definition of $Z(2)$, assuming $P\left[\tilde{V}_{m}=s_{0, .}^{.}\right]>0$, which implies $P\left[\hat{X}_{m}=r\right]>0$ :

$$
\begin{aligned}
\tilde{v}(n, k) & =P\left[\hat{X}_{m+n}=f \wedge \hat{X}_{m+1: m+n-1} \in S^{\prime} \wedge \#\left(\hat{X}_{m: m+n-1} \in U_{S}\right)=k \mid \hat{X}_{m}=r\right] \\
& =P\left[\hat{X}_{n}^{\prime}=f \wedge \hat{X}_{1: n-1}^{\prime} \in S^{\prime} \wedge \#\left(\hat{X}_{0: n-1}^{\prime} \in U_{S}\right)=k\right] \\
& =P\left[Z_{n}=f \wedge Z_{0: n-1} \in S \wedge \#\left(Z_{0: n-1} \in U_{S}\right)=k\right]=\sum_{i \in S} \pi_{i}(n-1, k) P_{i, f} .
\end{aligned}
$$

On the other hand, using the definition of $\hat{v}(n, k)$ (A.9), considering the state transition diagram of $\hat{V}$ and using result (1), (A.11) and (A.12), assuming $P\left[\hat{V}_{m}=\dot{s_{0,} .}\right]>0$ :

$$
\begin{aligned}
\hat{v}(n, k) & =P\left[\hat{V}_{m+n}=f \wedge \hat{V}_{n+1: m+n-1} \in{ }^{V} S^{\prime} \wedge \#\left(\hat{V}_{m: m+n-1} \in{ }^{V} U_{S}\right)=k \mid \hat{V}_{m}=\dot{s_{0, .}}\right] \\
= & P\left[\hat{V}_{m+n}=f \mid \hat{V}_{m+n-1}=s_{n-1, k}^{u}\right] P\left[\hat{V}_{m+n-1}=s_{n-1, k}^{u} \mid \hat{V}_{m}=\dot{s_{0, .}}\right] \\
& +P\left[\hat{V}_{m+n}=f \mid \hat{V}_{m+n-1}=s_{n-1, k}^{d}\right] P\left[\hat{V}_{m+n-1}=s_{n-1, k}^{d} \mid \hat{V}_{m}=\dot{s_{0,} .}\right] \\
= & v_{n-1, k}^{u} \hat{w}^{u}(n-1, k)+v_{n-1, k}^{d} \hat{w}^{d}(n-1, k) \\
= & \frac{\sum_{i \in U_{S}} \pi_{i}(n-1, k) P_{i, f}}{\sum_{i \in U_{S}} \pi_{i}(n-1, k)} \tilde{w}^{u}(n-1, k)+\frac{\sum_{i \in D_{S}} \pi_{i}(n-1, k) P_{i, f}}{\sum_{i \in D_{S}} \pi_{i}(n-1, k)} \tilde{w}^{d}(n-1, k) \\
= & \sum_{i \in U_{S}} \pi_{i}(n-1, k) P_{i, f}+\sum_{i \in D_{S}} \pi_{i}(n-1, k) P_{i, f}=\sum_{i \in S} \pi_{i}(n-1, k) P_{i, f} .
\end{aligned}
$$

Proof of (3) $(\hat{q}(n, k)=\tilde{q}(n, k))$. The developments are very similar to those of the proof of result (2). Assuming $P\left[\tilde{V}_{m}=s_{0,}^{\cdot}\right]>0$, which implies $P\left[\hat{X}_{m}=r\right]>0$ :

$$
\begin{aligned}
\tilde{q}(n, k) & =P\left[\hat{X}_{m+n}=r \wedge \hat{X}_{m+1: m+n-1} \in S^{\prime} \wedge \#\left(\hat{X}_{m: m+n-1} \in U_{S}\right)=k \mid \hat{X}_{m}=r\right] \\
& =P\left[\hat{X}_{n}^{\prime}=r \wedge \hat{X}_{1: n-1}^{\prime} \in S^{\prime} \wedge \#\left(\hat{X}_{0: n-1}^{\prime} \in U_{S}\right)=k\right] \\
& =P\left[Z_{n}=a \wedge Z_{0: n-1} \in S \wedge \#\left(Z_{0: n-1} \in U_{S}\right)=k\right]=\sum_{i \in S} \pi_{i}(n-1, k) P_{i, r},
\end{aligned}
$$


and, assuming $P\left[\hat{V}_{m}=s_{0, \cdot}^{\cdot}\right]>0$ :

$$
\begin{aligned}
\hat{q}(n, k)= & P\left[\hat{V}_{m+n}=\dot{s_{0, \cdot}} \wedge \hat{V}_{n+1: m+n-1} \in{ }^{V} S^{\prime} \wedge \#\left(\hat{V}_{m: m+n-1} \in{ }^{V} U_{S}\right)=k \mid \hat{V}_{m}=\dot{s_{0, .}}\right] \\
= & P\left[\hat{V}_{m+n}=\dot{s_{0, \cdot}} \mid \hat{V}_{m+n-1}=s_{n-1, k}^{u}\right] P\left[\hat{V}_{m+n-1}=s_{n-1, k}^{u} \mid \hat{V}_{m}=\dot{s_{0, .}}\right] \\
& +P\left[\hat{V}_{m+n}=\dot{s_{0, \cdot}} \mid \hat{V}_{m+n-1}=s_{n-1, k}^{d}\right] P\left[\hat{V}_{m+n-1}=s_{n-1, k}^{d} \mid \hat{V}_{m}=\dot{s_{0, .}}\right] \\
= & q_{n-1, k}^{u} \hat{w}^{u}(n-1, k)+q_{n-1, k}^{d} \hat{w}^{d}(n-1, k) \\
= & \frac{\sum_{i \in U_{S}} \pi_{i}(n-1, k) P_{i, r}}{\sum_{i \in U_{S}} \pi_{i}(n-1, k)} \tilde{w}^{u}(n-1, k)+\frac{\sum_{i \in D_{S}} \pi_{i}(n-1, k) P_{i, r}}{\sum_{i \in D_{S}} \pi_{i}(n-1, k)} \tilde{w}^{d}(n-1, k) \\
= & \sum_{i \in U_{S}} \pi_{i}(n-1, k) P_{i, r}+\sum_{i \in D_{S}} \pi_{i}(n-1, k) P_{i, r}=\sum_{i \in S} \pi_{i}(n-1, k) P_{i, r} .
\end{aligned}
$$

Proof of (4) $\left(\hat{w}^{\prime \prime}(n, k)=\tilde{w}^{\prime \prime}(n, k), \hat{w}^{\prime d}(n, k)=\tilde{w}^{\prime d}(n, k)\right)$. The proof is by induction on $n$. For the base case, $n=0$, we trivially have

$$
\begin{aligned}
& \hat{w}^{\prime u}(0,0)=\tilde{w}^{\prime u}(0,0)=0, \\
& \hat{w}^{\prime u}(0,1)=\tilde{w}^{\prime u}(0,1)=\alpha_{U_{S}^{\prime}} \\
& \hat{w}^{\prime d}(0,0)=\tilde{w}^{\prime d}(0,0)=\alpha_{D_{S}^{\prime}}, \\
& \hat{w}^{\prime u}(0,1)=\tilde{w}^{\prime u}(0,1)=0 .
\end{aligned}
$$

Let us proceed with the induction step. First, using the definitions of $\tilde{w}^{\prime u}(n, k)(\mathrm{A} .8), \tilde{V}(21)$, and $Z^{\prime}(3)$ :

$$
\begin{aligned}
\tilde{w}^{\prime u}(n, k) & =P\left[\hat{X}_{0: n} \in S^{\prime} \wedge \#\left(\hat{X}_{0: n} \in U_{S}^{\prime}\right)=k \wedge \hat{X}_{n} \in U_{S}^{\prime}\right] \\
& =P\left[\#\left(Z_{0: n}^{\prime} \in U_{S}^{\prime}\right)=k \wedge Z_{n}^{\prime} \in U_{S}^{\prime}\right]=\sum_{i \in U_{S}^{\prime}} \pi_{i}^{\prime}(n, k) .
\end{aligned}
$$

Similarly:

$$
\begin{aligned}
\tilde{w}^{\prime d}(n, k) & =P\left[\hat{X}_{0: n} \in S^{\prime} \wedge \#\left(\hat{X}_{0: n} \in U_{S}^{\prime}\right)=k \wedge \hat{X}_{n} \in D_{S}^{\prime}\right] \\
& =P\left[\#\left(Z_{0: n}^{\prime} \in U_{S}^{\prime}\right)=k \wedge Z_{n}^{\prime} \in D_{S}^{\prime}\right]=\sum_{i \in D_{S}^{\prime}} \pi_{i}^{\prime}(n, k) .
\end{aligned}
$$

On the other hand, considering the state transition diagram of $\hat{V}$ and using the induction hypothesis, (A.13), (A.14), (13), and (14):

$$
\begin{aligned}
\hat{w}^{\prime u}(n+1, k) & =P\left[\hat{V}_{n+1}=s_{n+1, k}^{\prime u}\right] \\
& =P\left[\hat{V}_{n+1}=s_{n+1, k}^{\prime u} \mid \hat{V}_{n}=s_{n, k-1}^{\prime u}\right] P\left[\hat{V}_{n}=s_{n, k-1}^{\prime u}\right]
\end{aligned}
$$




$$
\begin{aligned}
& +P\left[\hat{V}_{n+1}=s_{n+1, k}^{\prime u} \mid \hat{V}_{n}=s_{n, k-1}^{\prime d}\right] P\left[\hat{V}_{n}=s_{n, k-1}^{\prime d}\right] \\
& =w_{n, k-1}^{\prime u u} \hat{w}^{\prime u}(n, k-1)+w_{n, k-1}^{\prime d u} \hat{w}^{\prime d}(n, k-1) \\
& =\frac{\sum_{i \in U_{S}^{\prime}} \pi_{i}^{\prime}(n, k-1) P_{i, U_{S}^{\prime}}}{\sum_{i \in U_{S}^{\prime}} \pi_{i}^{\prime}(n, k-1)} \tilde{w}^{\prime u}(n, k-1)+\frac{\sum_{i \in D_{S}^{\prime}} \pi_{i}^{\prime}(n, k-1) P_{i, U_{S}^{\prime}}}{\sum_{i \in D_{S}^{\prime}} \pi_{i}^{\prime}(n, k-1)} \tilde{w}^{\prime d}(n, k-1) \\
& =\sum_{i \in U_{S}^{\prime}} \pi_{i}^{\prime}(n, k-1) P_{i, U_{S}^{\prime}}+\sum_{i \in D_{S}^{\prime}} \pi_{i}^{\prime}(n, k-1) P_{i, U_{S}^{\prime}}=\sum_{i \in S^{\prime}} \pi_{i}^{\prime}(n, k-1) P_{i, U_{S}^{\prime}} \\
& =\sum_{i \in U_{S}^{\prime}} \pi_{i}^{\prime}(n+1, k)=\tilde{w}^{\prime \prime}(n+1, k), \\
& \hat{w}^{\prime d}(n+1, k)=P\left[\hat{V}_{n+1}=s_{n+1, k}^{\prime d}\right] \\
& =P\left[\hat{V}_{n+1}=s_{n+1, k}^{\prime d} \mid \hat{V}_{n}=s_{n, k}^{\prime u}\right] P\left[\hat{V}_{n}=s_{n, k}^{\prime u}\right] \\
& +P\left[\hat{V}_{n+1}=s_{n+1, k}^{\prime d} \mid \hat{V}_{n}=s_{n, k}^{\prime d}\right] P\left[\hat{V}_{n}=s_{n, k}^{\prime d}\right] \\
& =w_{n, k}^{\prime u d} \hat{w}^{\prime u}(n, k)+w_{n, k}^{\prime d d} \hat{w}^{\prime d}(n, k) \\
& =\frac{\sum_{i \in U_{S}^{\prime}} \pi_{i}^{\prime}(n, k) P_{i, D_{S}^{\prime}}}{\sum_{i \in U_{S}^{\prime}} \pi_{i}^{\prime}(n, k)} \tilde{w}^{\prime u}(n, k)+\frac{\sum_{i \in D_{S}^{\prime}} \pi_{i}^{\prime}(n, k) P_{i, D_{S}^{\prime}}}{\sum_{i \in D_{S}^{\prime}} \pi_{i}^{\prime}(n, k)} \tilde{w}^{\prime d}(n, k) \\
& =\sum_{i \in U_{S}^{\prime}} \pi_{i}^{\prime}(n, k) P_{i, D_{S}^{\prime}}+\sum_{i \in D_{S}^{\prime}} \pi_{i}^{\prime}(n, k) P_{i, D_{S}^{\prime}}=\sum_{i \in S^{\prime}} \pi_{i}^{\prime}(n, k) P_{i, D_{S}^{\prime}} \\
& =\sum_{i \in D_{S}^{\prime}} \pi_{i}^{\prime}(n+1, k)=\tilde{w}^{\prime d}(n+1, k)
\end{aligned}
$$

Proof of $(5)\left(\hat{v}^{\prime}(n, k)=\tilde{v}^{\prime}(n, k)\right)$. Using the definitions of $\tilde{v}^{\prime}(n, k)(\mathrm{A} .7), \tilde{V}(21)$ and $Z^{\prime}(3)$ :

$$
\begin{aligned}
\tilde{v}^{\prime}(n, k) & =P\left[\hat{X}_{n}=f \wedge \hat{X}_{0: n-1} \in S^{\prime} \wedge \#\left(\hat{X}_{0: n-1} \in U_{S}^{\prime}\right)=k\right] \\
& =P\left[Z_{n}^{\prime}=f \wedge Z_{0: n-1}^{\prime} \in S^{\prime} \wedge \#\left(Z_{0: n-1}^{\prime} \in U_{S}^{\prime}\right)=k\right]=\sum_{i \in S^{\prime}} \pi_{i}^{\prime}(n-1, k) P_{i, f} .
\end{aligned}
$$

On the other hand, using the definition of $\hat{v}^{\prime}(n, k)$ (A.10), considering the state transition diagram of $\hat{V}$ and using result (4), (A.13) and (A.14):

$$
\begin{aligned}
\hat{v}^{\prime}(n, k) & =P\left[\hat{V}_{n}=f \wedge \hat{V}_{0: n-1} \in{ }^{V} S^{\prime} \wedge \#\left(\hat{V}_{0: n-1} \in{ }^{V} U_{S}^{\prime}\right)=k\right] \\
& =P\left[\hat{V}_{n}=f \mid \hat{V}_{n-1}=s_{n-1, k}^{\prime u}\right] P\left[\hat{V}_{n-1}=s_{n-1, k}^{\prime u}\right]
\end{aligned}
$$




$$
\begin{aligned}
& +P\left[\hat{V}_{n}=f \mid \hat{V}_{n-1}=s_{n-1, k}^{\prime d}\right] P\left[\hat{V}_{n-1}=s_{n-1, k}^{\prime d}\right] \\
= & v_{n-1, k}^{\prime u} \hat{w}^{\prime u}(n-1, k)+v_{n-1, k}^{\prime d} \hat{w}^{\prime d}(n-1, k) \\
= & \frac{\sum_{i \in U_{S}^{\prime}} \pi_{i}^{\prime}(n-1, k) P_{i, f}}{\sum_{i \in U_{S}^{\prime}} \pi_{i}^{\prime}(n-1, k)} \tilde{w}^{\prime u}(n-1, k)+\frac{\sum_{i \in D_{S}^{\prime}} \pi_{i}^{\prime}(n-1, k) P_{i, f}}{\sum_{i \in D_{S}^{\prime}} \pi_{i}^{\prime}(n-1, k)} \tilde{w}^{\prime d}(n-1, k) \\
= & \sum_{i \in U_{S}^{\prime}} \pi_{i}^{\prime}(n-1, k) P_{i, f}+\sum_{i \in D_{S}^{\prime}} \pi_{i}^{\prime}(n-1, k) P_{i, f}=\sum_{i \in S^{\prime}} \pi_{i}^{\prime}(n-1, k) P_{i, f} .
\end{aligned}
$$

Proof of $(6)\left(\hat{q}^{\prime}(n, k)=\tilde{q}^{\prime}(n, k)\right)$. The developments are very similar to those of the proof of result (5). We have:

$$
\begin{aligned}
\tilde{q}^{\prime}(n, k) & =P\left[\hat{X}_{n}=r \wedge \hat{X}_{0: n-1} \in S^{\prime} \wedge \#\left(\hat{X}_{0: n-1} \in U_{S}^{\prime}\right)=k\right] \\
& =P\left[Z_{n}^{\prime}=a \wedge Z_{0: n-1}^{\prime} \in S^{\prime} \wedge \#\left(Z_{0: n-1}^{\prime} \in U_{S}^{\prime}\right)=k\right]=\sum_{i \in S^{\prime}} \pi_{i}^{\prime}(n-1, k) P_{i, r},
\end{aligned}
$$

and:

$$
\begin{aligned}
\hat{q}^{\prime}(n, k)= & P\left[\hat{V}_{n}=\dot{s_{0, \cdot}} \wedge \hat{V}_{0: n-1} \in{ }^{V} S^{\prime} \wedge \#\left(\hat{V}_{0: n-1} \in{ }^{V} U_{S}^{\prime}\right)=k\right] \\
= & P\left[\hat{V}_{n}=\dot{s_{0,} \cdot \mid} \hat{V}_{n-1}=s_{n-1, k}^{\prime u}\right] P\left[\hat{V}_{n-1}=s_{n-1, k}^{\prime u}\right] \\
& +P\left[\hat{V}_{n}=\dot{s_{0, \cdot}} \cdot \hat{V}_{n-1}=s_{n-1, k}^{\prime d}\right] P\left[\hat{V}_{n-1}=s_{n-1, k}^{\prime d}\right] \\
= & q_{n-1, k}^{\prime u} \hat{w}^{\prime u}(n-1, k)+q_{n-1, k}^{\prime d} \hat{w}^{\prime d}(n-1, k) \\
= & \frac{\sum_{i \in U_{S}^{\prime}} \pi_{i}^{\prime}(n-1, k) P_{i, r}}{\sum_{i \in U_{S}^{\prime}} \pi_{i}^{\prime}(n-1, k)} \tilde{w}^{\prime u}(n-1, k)+\frac{\sum_{i \in D_{S}^{\prime}} \pi_{i}^{\prime}(n-1, k) P_{i, r}}{\sum_{i \in D_{S}^{\prime}} \pi_{i}^{\prime}(n-1, k)} \tilde{w}^{\prime d}(n-1, k) \\
= & \sum_{i \in U_{S}^{\prime}} \pi_{i}^{\prime}(n-1, k) P_{i, r}+\sum_{i \in D_{S}^{\prime}} \pi_{i}^{\prime}(n-1, k) P_{i, r}=\sum_{i \in S^{\prime}} \pi_{i}^{\prime}(n-1, k) P_{i, r} \cdot \square
\end{aligned}
$$

Proof of Proposition 4. We will start by considering the case $\alpha_{S^{\prime}}>0$. Let $c_{C, K, L}^{\prime}(k)$ be the probability that $\hat{V}_{C, K, L}$ has entered state $b$ through a state $s_{n, K}^{u}$ or a state $s_{n, K}^{d}$ after no more than $k$ visits to states $s_{n, l}^{u}, s_{n, l}^{\prime u}, n>0$. Trivially, $c_{C, K, L}(k) \leqslant c_{C, K, L}^{\prime}(k)$.

Consider the DTMC $W_{C, K, L}$ with the state transition diagram depicted in Fig. 15, initial probability distribution $P\left[\left(W_{C, K, L}\right)_{0}=s_{0}\right]=\alpha_{r}, P\left[\left(W_{C, K, L}\right)_{0}=s_{0}^{\prime}\right]=\alpha_{S^{\prime}}, P\left[\left(W_{C, K, L}\right)_{0}=f\right]=\alpha_{f}$, and $P\left[\left(W_{C, K, L}\right)_{0}\right.$ $=i]=0, i \notin\left\{s_{0}, s_{0}^{\prime}, f\right\}$, and transition probabilities chosen so that (the selections are always 


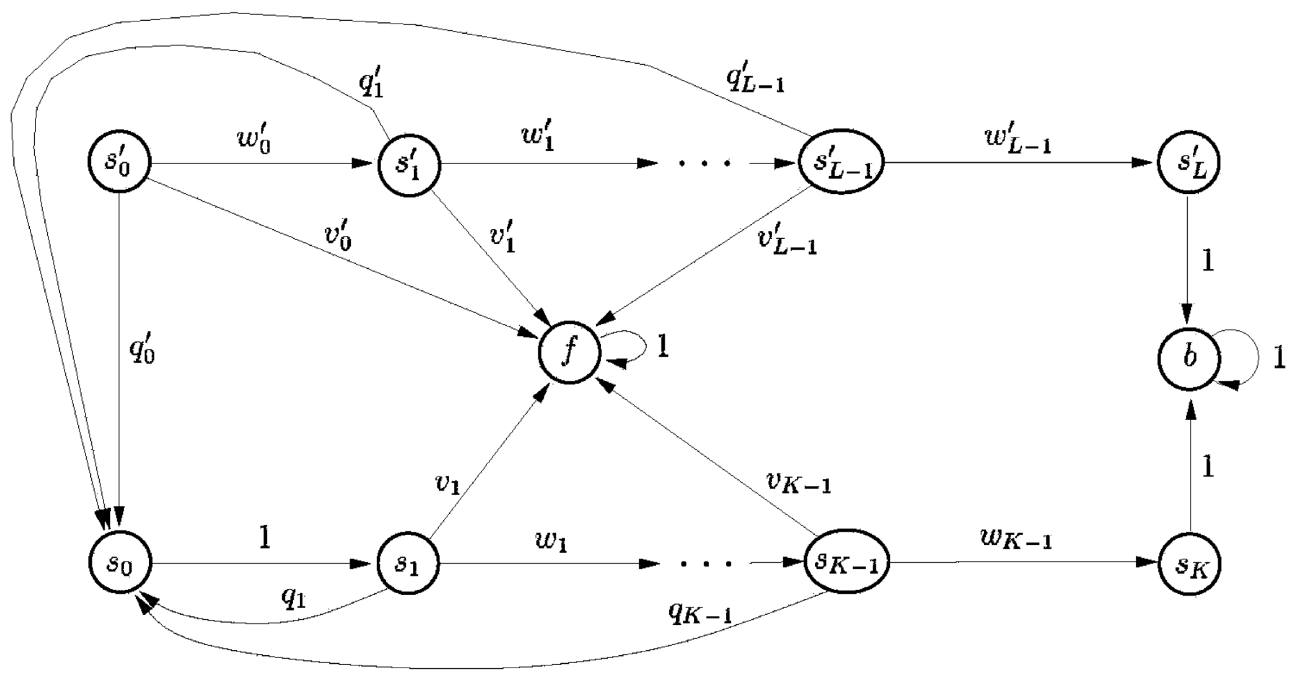

Fig. 15. State transition diagram of the DTMC $W_{C, K, L}$.

possible):

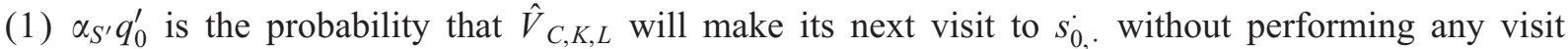
to a state $s_{n, k}^{\prime u}, n>0$.

(2) $\alpha_{S^{\prime}} \prod_{i=0}^{k-1} w_{i}^{\prime} q_{k}^{\prime}, 1 \leqslant k \leqslant L-1$ is the probability that $\hat{V}_{C, K, L}$ will make its next visit to $s_{0,}$. after performing exactly $k$ visits to states $s_{n, k}^{\prime \prime}, n>0$.

(3) $\alpha_{S^{\prime}} \prod_{i=0}^{L-1} w_{i}^{\prime}$ is the probability that $\hat{V}_{C, K, L}$ will enter a state $s_{n, L}^{\prime u}$ or $s_{n, L}^{\prime d}$ (after performing $L-2$ or $L-1$ visits to states $\left.s_{n, k}^{\prime u}, n>0\right)$.

(4) $\prod_{i=1}^{k-1} w_{i} q_{k}, 1 \leqslant k \leqslant K-1$ is the probability that, starting at $s_{0,}^{\cdot}, \hat{V}_{C, K, L}$ will make its next visit to $s_{0,}$. after performing exactly $k$ visits to states $s_{n, k}^{u}, n>0$.

(5) $\prod_{i=1}^{K-1} w_{i}$ is the probability that, starting at $s_{0, .}, \hat{V}_{C, K, L}$ will enter a state $s_{n, K}^{u}$ or $s_{n, K}^{d}$ (after performing $K-2$ or $K-1$ visits to states $s_{n, k}^{u}, n>0$ ) without performing any further visit to state $s_{0, \cdot}$.

Let $c_{C, K, L}^{\prime \prime}(k)$ be the probability that $W_{C, K, L}$ will enter $b$ through state $s_{K}$ after no more than $k$ visits to states $s_{n}^{\prime}, s_{n}, n>0$. By definition of $W_{C, K, L}$ and since state $s_{K}$ is visited just once when $W_{C, K, L}$ enters $b$ through $s_{K}$ and, in that case, $s_{L}^{\prime}$ is not visited, we have $c_{C, K, L}^{\prime}(k) \leqslant c_{C, K, L}^{\prime \prime}(k+1)$. Let $\tilde{W}_{C, K, L}$ be the DTMC obtained from $W_{C, K, L}$ by substituting states $s_{0}, s_{0}^{\prime}$ by instantaneous switches, in a way similar as to how vanishing markings are eliminated in GSPNs [20]. Fig. 16 depicts the state transition diagram of $\tilde{W}_{C, K, L}$. The important point is that the transition probabilities of $\tilde{W}_{C, K, L}$ from $s_{k}$ to $s_{k+1}$ are identical to the respective transition probabilities of $W_{C, K, L}$. Also, $P\left[\left(\tilde{W}_{C, K, L}\right)_{0} \in\right.$ $\left.\left\{s_{1}, s_{1}^{\prime}\right\}\right] \leqslant P\left[\left(W_{C, K, L}\right)_{0}=s_{0}\right]+P\left[\left(W_{C, K, L}\right)_{0}=s_{0}^{\prime}\right]=\alpha_{r}+\alpha_{S^{\prime}}=\alpha_{S}$. It is clear that $c_{C, K, L}^{\prime \prime}(k)$ is the probability that $\tilde{W}_{C, K, L}$ will enter state $b$ through state $s_{K}$ after no more than $k$ visits to states $s_{n}^{\prime}$, $s_{n}, n>0$, and, therefore, $c_{C, K, L}^{\prime \prime}(k)$ is the probability that $\tilde{W}_{C, K, L}$ will have entered state $b$ through state $s_{K}$ by step $k$. Then, using Proposition 2 of [13], with the DTMC $\hat{V}_{K, L}$ of that proposition 


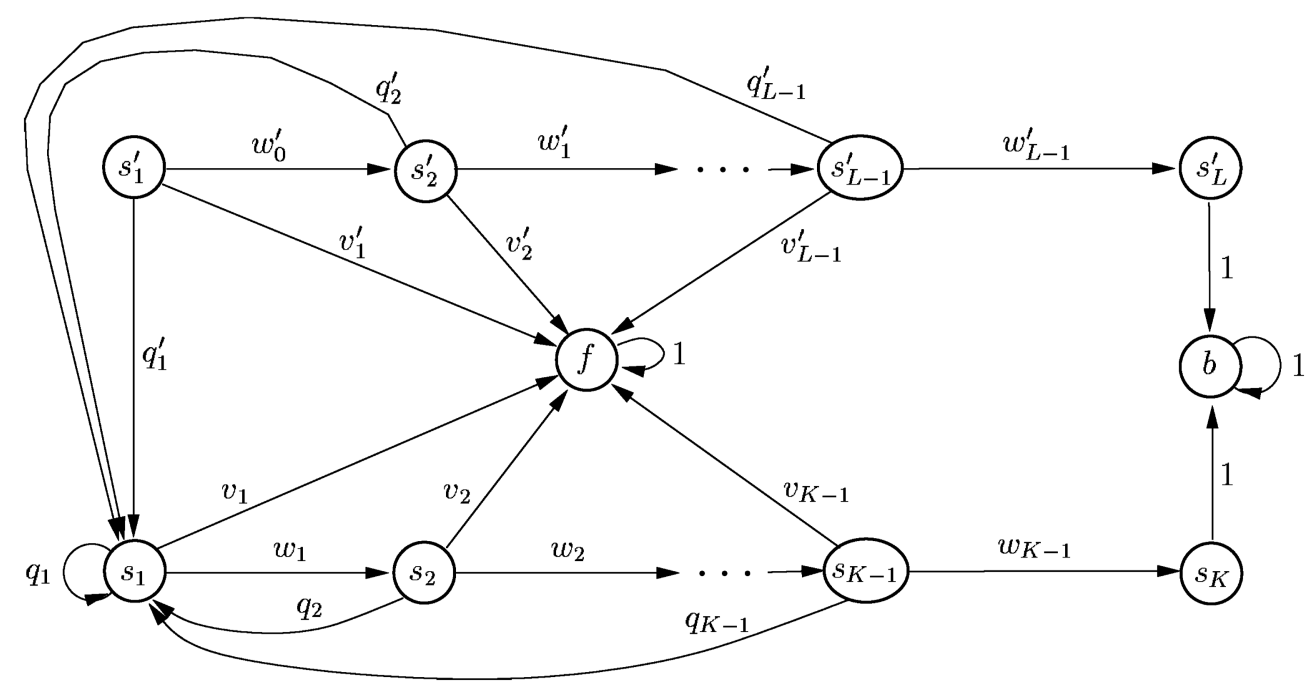

Fig. 16. State transition diagram of the DTMC $\tilde{W}_{C, K, L}$.

being $\tilde{W}_{C, K, L}$ :

$$
c_{C, K, L}^{\prime \prime}(k) \leqslant I_{k>K-1} P\left[\left(\tilde{W}_{C, K, L}\right)_{0} \in\left\{s_{1}, s_{1}^{\prime}\right\}\right](k-K+1) \prod_{i=1}^{K-1} w_{i} .
$$

But, being $\prod_{i=1}^{K-1} w_{i}$ the probability that, starting at $s_{0, .}^{\cdot}, \hat{V}_{C, K, L}$ will enter a state $s_{n, K}^{u}$ or $s_{n, K}^{d}$ without performing any further visit to $s_{0,}^{\cdot}, \prod_{i=1}^{K-1} w_{i}=\sum_{n=K-1}^{K+C-1} \sum_{i \in S} \pi_{i}(n, K)$. Then, using $P\left[\left(\tilde{W}_{C, K, L}\right)_{0} \in\right.$ $\left.\left\{s_{1}, s_{1}^{\prime}\right\}\right] \leqslant \alpha_{S}$, we have

$$
c_{C, K, L}(k) \leqslant c_{C, K, L}^{\prime}(k) \leqslant c_{C, K, L}^{\prime \prime}(k+1) \leqslant I_{k} \geqslant K-1 \alpha_{S}(k-K+2) a_{C}(K),
$$

with $a_{C}(K)=\sum_{n=K-1}^{K+C-1} \sum_{i \in S} \pi_{i}(n, K)$.

The fact that, for the case $\alpha_{S^{\prime}}=0, c_{C, K}(k) \leqslant I_{k} \geqslant K-1 \alpha_{S}(k-K+2) a_{C}(K)$ can be proved similarly.

Proof of Theorem 4. Let $\pi_{i}(n)=P\left[Z_{n}=i\right], i \in S$ and $\pi_{i}^{\prime}(n)=P\left[Z_{n}^{\prime}=i\right], i \in S^{\prime}$. We have

$$
a_{C}(n)=\sum_{m=n-1}^{n+C-1} \sum_{i \in S} \pi_{i}(m, n) \leqslant(C+1) P(n),
$$

where $P(n)$ is the probability that $Z$ will make $n$ or more visits to states in $U_{S}$ before entering the absorbing state $a$, which is trivially not greater than the probability that $Z$ will make $n$ or more visits to states in $S$ before entering state $a$, which is equal to $a(n)=\sum_{i \in S} \pi_{i}(n)$. Then, we have $a_{C}(n) \leqslant(C+1) a(n)$. It can be proved similarly, for the case $\alpha_{S^{\prime}}>0$, that $a_{C}^{\prime}(n) \leqslant(C+1) a^{\prime}(n)$, with $a^{\prime}(n)=\sum_{i \in S^{\prime}} \pi_{i}^{\prime}(n)$. The states $i \in S$ are transient in $Z$ and the states $i \in S^{\prime}$ are transient in 
$Z^{\prime}$. Then [21, Theorem 4.3], $a(n)$ and $a^{\prime}(n)$ decrease geometrically fast, i.e. there exist $B, B^{\prime}>0$, $0<b, b^{\prime}<1$ and $n_{0}, n_{0}^{\prime} \geqslant 0$ such that

$$
\begin{aligned}
& a(n) \leqslant B b^{n}, \quad n \geqslant n_{0}, \\
& a^{\prime}(n) \leqslant B^{\prime} b^{\prime n}, \quad n \geqslant n_{0}^{\prime} .
\end{aligned}
$$

The model truncation error upper bounds used to select $K$ and $L$ can be upper bounded, using $K, L \geqslant 2$, as follows:

$$
\begin{aligned}
& \alpha_{S} a_{C}(K) \sum_{k=K}^{\infty}(k-K+2) \mathrm{e}^{-\Lambda_{U} t} \frac{\left(\Lambda_{U} t\right)^{k}}{k !}=\alpha_{S} a_{C}(K) \sum_{k=2}^{\infty} k \mathrm{e}^{-\Lambda_{U} t} \frac{\left(\Lambda_{U} t\right)^{K+k-2}}{(K+k-2) !} \\
& \leqslant \alpha_{S} a_{C}(K) \sum_{k=2}^{\infty} \mathrm{e}^{-\Lambda_{U} t} \frac{\left(\Lambda_{U} t\right)^{K+k-2}}{(K+k-3) !}=\alpha_{S} a_{C}(K) \Lambda_{U} t \sum_{k=2}^{\infty} \mathrm{e}^{-\Lambda_{U} t} \frac{\left(\Lambda_{U} t\right)^{K+k-3}}{(K+k-3) !} \\
& \quad=\alpha_{S} a_{C}(K) \Lambda_{U} t \sum_{k=K-1}^{\infty} \mathrm{e}^{-\Lambda_{U} t} \frac{\left(\Lambda_{U} t\right)^{k}}{k !}<\alpha_{S} a_{C}(K) \Lambda_{U} t \leqslant \alpha_{S}(C+1) a(K) \Lambda_{U} t, \\
& a_{C}^{\prime}(L) \sum_{k=L}^{\infty} \mathrm{e}^{-\Lambda_{U} t} \frac{\left(\Lambda_{U} t\right)^{k}}{k !}<a_{C}^{\prime}(L) \leqslant(C+1) a^{\prime}(L) .
\end{aligned}
$$

We will show next that $C=O\left(\Lambda_{U} t / \varepsilon\right)$. Since we are in the case $U_{S}^{\prime} \neq \emptyset$, the exact value of $C$ is

$$
C=\min \left\{c \geqslant 1: \sum_{k=c+1}^{\infty} \mathrm{e}^{-\Lambda t q} \frac{(\Lambda t q)^{k}}{k !} \leqslant \frac{\varepsilon}{4}\right\} .
$$

Let $Q_{q}(t)$ be a Poisson process with arrival rate $\Lambda q$. Then, $\sum_{k=c+1}^{\infty} \mathrm{e}^{-\Lambda t q}(\Lambda t q)^{k} / k !=P\left[Q_{q}(t)>c\right]$. For $c \geqslant 1$, using

$$
E\left[Q_{q}(t)\right]=\Lambda q t=P\left[Q_{q}(t) \leqslant c\right] E\left[Q_{q}(t) \mid Q_{q}(t) \leqslant c\right]+P\left[Q_{q}(t)>c\right] E\left[Q_{q}(t) \mid Q_{q}(t)>c\right],
$$

$E\left[Q_{q}(t) \mid Q_{q}(t) \leqslant c\right]>0$ and $E\left[Q_{q}(t) \mid Q_{q}(t)>c\right]>c$, we obtain

$$
P\left[Q_{q}(t)>c\right]<\frac{\Lambda t q}{c} .
$$

Then, $C$ is upper bounded by $\lfloor x\rfloor+1$, where $x$ satisfies

$$
\begin{aligned}
& \frac{\Lambda t q}{x}=\frac{\varepsilon}{4}, \\
& x=\frac{4 \Lambda t q}{\varepsilon} .
\end{aligned}
$$

Since, for a given CTMC, $\Lambda$ and $\Lambda_{U}$ are constants and, in our analysis, $q$ is a constant, this shows that $x$ and $C$ are $O\left(\Lambda_{U} t / \varepsilon\right)$. 
Consider the case $\alpha_{S^{\prime}}>0$. Using (A.17), the required $K$ is upper bounded by the minimum integer $n$ satisfying $\alpha_{S}(C+1) a(n) \Lambda_{U} t \leqslant \varepsilon / 8$. Using (A.15), that integer is not greater than $\max \left\{n_{0}, l\right\}$, where $l$ is the minimum integer satisfying $\alpha_{S}(C+1) B b^{l} \Lambda_{U} t \leqslant \varepsilon / 8$. Then, to prove that the required $K$ is $O\left(\log \left(\Lambda_{U} t / \varepsilon\right)\right)$, it suffices to prove that $l$ is $O\left(\log \left(\Lambda_{U} t / \varepsilon\right)\right)$. But $l$ is not greater than $\lfloor x\rfloor+1$, where $x$ satisfies

$$
\alpha_{S}(C+1) B b^{x} \Lambda_{U} t=\frac{\varepsilon}{8} .
$$

Taking logarithms:

$$
\begin{aligned}
& \log \alpha_{S}+\log (C+1)+\log B+x \log b+\log \left(\Lambda_{U} t\right)=\log \left(\frac{\varepsilon}{8}\right), \\
& x=\frac{\log \left(8 \Lambda_{U} t / \varepsilon\right)+\log (C+1)+\log \alpha_{S}+\log B}{\log (1 / b)} .
\end{aligned}
$$

This together with $\log (1 / b)>0$ (because $0<b<1$ ) and $C=O\left(\Lambda_{U} t / \varepsilon\right)$ shows that $x, l$ and the required $K$ are $O\left(\log \left(\Lambda_{U} t / \varepsilon\right)\right)$. Using (A.18), the required $L$ is upper bounded by the minimum integer $n$ satisfying $(C+1) a^{\prime}(n) \leqslant \varepsilon / 8$. Using (A.16), that integer is not greater than $\max \left\{n_{0}^{\prime}, l\right\}$, where $l$ is the minimum integer satisfying $(C+1) B^{\prime} b^{\prime l} \leqslant \varepsilon / 8$. Then, to prove that the required $L$ is $O\left(\log \left(\Lambda_{U} t / \varepsilon\right)\right)$, it suffices to prove that $l$ is $O\left(\log \left(\Lambda_{U} t / \varepsilon\right)\right)$. But $l$ is not greater than $\lfloor x\rfloor+1$, where $x$ satisfies

$$
(C+1) B^{\prime} b^{\prime x}=\frac{\varepsilon}{8} .
$$

Taking logarithms:

$$
\begin{aligned}
& \log (C+1)+\log B^{\prime}+x \log b^{\prime}=\log \left(\frac{\varepsilon}{8}\right), \\
& x=\frac{\log (8 / \varepsilon)+\log (C+1)+\log B^{\prime}}{\log \left(1 / b^{\prime}\right)} .
\end{aligned}
$$

This together with $\log \left(1 / b^{\prime}\right)>0$ (because $\left.0<b^{\prime}<1\right)$ and $C=O\left(\Lambda_{U} t / \varepsilon\right)$ shows that $x, l$ and the required $L$ are $O\left(\log \left(\Lambda_{U} t / \varepsilon\right)\right)$.

The proof that, for the case $\alpha_{S^{\prime}}=0$, the required $K$ is $O\left(\log \left(\Lambda_{U} t / \varepsilon\right)\right)$ follows the proof that, for the case $\alpha_{S^{\prime}}>0$, the required $K$ is $O\left(\log \left(\Lambda_{U} t / \varepsilon\right)\right)$ with $\varepsilon$ replaced by $2 \varepsilon$ (because the allocated model truncation error is $\varepsilon / 4$ instead of $\varepsilon / 8)$.

\section{References}

[1] Barlow RE, Hunter LC. Reliability analysis of a one-unit system. Operations Research 1961;9(2):200-8.

[2] Goyal A, Tantawi AN. A measure of guaranteed availability and its numerical evaluation. IEEE Transactions on Computers 1988;C-37(1):25-32.

[3] Ross S. Stochastic processes. New York: Wiley; 1983.

[4] Rubino G, Sericola B. Interval availability analysis using operational periods. Performance Evaluation 1992;14: 257-72.

[5] Rubino G, Sericola B. Interval availability distribution computation. In: Proceedings of the 23rd IEEE International Symposium on Fault-Tolerant Computing (FTCS-23), 1993. p. 48-55. 
[6] Rubino G, Sericola B. Interval availability analysis using denumerable Markov processes: application to multiprocessor subject to breakdowns and repair. IEEE Transactions on Computers 1995;44(2):286-91.

[7] Sericola B. Closed form solution for the distribution of the total time spent in a subset of a homogeneous Markov process during a finite observation period. Journal of Applied Probability 1990;27:713-9.

[8] Sericola B. Occupation times in Markov processes. Communications in Statistics_-Stochastic Models 2000;16(5): 479-510.

[9] de Souza e Silva E, Gail HR. Calculating cumulative operational time distributions of repairable computer systems. IEEE Transactions on Computers 1986;C-35(4):322-32.

[10] de Souza e Silva E, Ochoa PM. State space exploration in Markov models. Performance Evaluation Review 1992;20(1):152-66.

[11] Kijima M. Markov processes for stochastic modeling. Cambridge: University Press; 1997.

[12] Çinlar E. Introduction to stochastic processes. Englewood Cliffs, NJ: Prentice-Hall; 1975.

[13] Carrasco JA. Transient analysis of large Markov models with absorbing states using regenerative randomization. Technical Report DMSD_99_2, Universitat Politècnica de Catalunya, January 1999, available at $\mathrm{ftp}: / / \mathrm{ftp}-\mathrm{eel} . u p c . e s /$ techreports.

[14] Carrasco JA. Computation of bounds for transient measures of large rewarded Markov models using regenerative randomization. Technical Report DMSD_99_4, Universitat Politècnica de Catalunya, May 1999, available at $\mathrm{ftp}: / / \mathrm{ftp}-\mathrm{eel} . u p c . e s /$ techreports, Computers and Operations Research, to appear.

[15] Knüsel L. Computation of the chi-square and Poisson distribution. SIAM Journal on Scientific and Statistical Computing 1986;7(3):1023-36.

[16] Neuts F. Matrix-geometric solutions in stochastic models. An algorithmic approach. New York: Dover; 1994.

[17] Bobbio A, Telek M. A benchmark for PH estimation algorithms: results for acyclic-PH. Communications in Statistics-Stochastic Models 1994;10(3):661-77.

[18] Varga RS. Matrix iterative analysis. Englewood Clifs, NJ: Prentice-Hall Inc.; 1962.

[19] Schott JR. Matrix analysis for statistics. New York: Wiley; 1997.

[20] Blakemore A. The cost of eliminating vanishing markings from generalized stochastic Petri nets. In: Proceedings of the Third IEEE International Workshop on Petri Nets and Performance Models (PNPM89), 1989. p. 85-92.

[21] Seneta E. Non-negative matrices and Markov chains. Springer Series in Statistics, 2nd ed. Springer, Berlin: 1981. 